UNIVERSIDADE DE SÃO PAULO

INSTITUTO DE FÍSICA

\title{
VIDROS DE SPIN COM INTERAÇÃO DE MULTISPINS EM CAMPOS
} ALEATÓRIOS

Luiz Ozorio de Oliveira Filho

Orientador: Prof. Dr. Carlos Seihiti Orii Yokoi

SBI-IFUSP

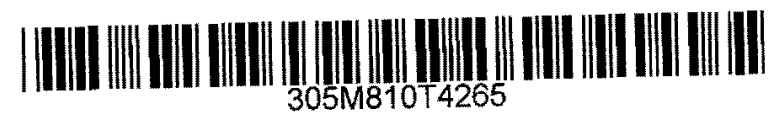

Tese apresentada ao Instituto de Física da

Universidade de São Paulo para obtenção

do título de Doutor em Ciências

BANCA EXAMINADORA

Prof. Dr. Carlos Seihiti Orii Yokoi (IFUSP)

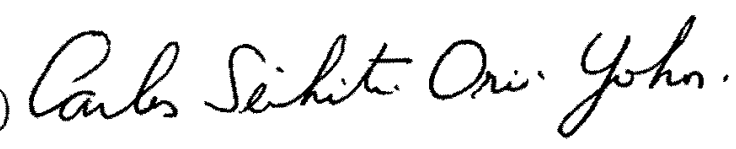

Prof. Dr. Francisco Alexandre da Costa (UFRN)

Prof. Dr. Lindberg Lima Gonçalves (UFC)

Prof. Dr. Sílvio Roberto de Azevedo Salinas (IFUSP)

Prof. Dr. Walter Felipe Wreszinski (IFUSP) 


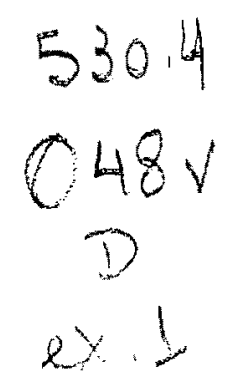

FICHA CATALOGRÁFICA

Preparada pelo Serviço de Biblioteca e Informação do Instituto de Física da Universidade de São Paulo

Oliveira Filho, Luiz Ozorio de

Vidros de spin com interação de multispins em campos aleatórios.

São Paulo - 2005.

Tese (Doutoramento) - Universidade de São Paulo Instituto de Física - Departamento de Física Geral

Orientador: Prof. Dr. Carlos Seihiti Orii Yokoi

Área de Concentração: Física da Matéria Condensada

Unitermos

1. Fisica da Matéria Condensada;

2. Sistemas Desordenados - Vidro de Spins;

3. Sistemas Desordenados -- Modelos de Energias Aleatórias;

4. Sistemas Desordenados - Campo Aleatório. 
Aos meus pais, Luís Osório e Marian Lima e meus irmãos, Jean Lima, Cláudia Maria e Rita Maria 


\section{Agradecimentos}

- Ao prof. Dr. Carlos Yokoi, pela orientação.

- Ao prof. Dr. Francisco Alexandre, por sua acolhida durante o período em que estive em Natal.

- Ao amigo André Vieira, por tudo que fez por mim desde minha chegada a São Paulo.

- Ao prof. José Chiappin por sua amizade, confiança e palavras de apoio nos momentos mais críticos.

- Ao prof. José Pimentel de Lima pelas boas conversas, amizade e por seu exemplo de perseverança.

- Aos amigos Alexandre, Ascânio, Firmeza, Egmídio Filho e Márcio André.

- À Márcia Silvani, ao Sérgio e Valdir por nos propiciarem um ambiente de trabalho bastante agradável e pelos inúmeros problemas solucionados.

- Ao apoio financeiro do CNPq e Capes e a Universidade Estadual Vale do Acaraú. 


\section{Resumo}

Estudamos o efeito do campo aleatório sobre um modelo de vidro de spin com interações de $p$ spins de alcance infinito e distribuição de probabilidade gaussiana. $O$ caso $p=2$ corresponde ao modelo de Sherrington-Kirkpatrick na presença de um campo aleatório. O caso $p \rightarrow \infty$ corresponde ao REM (Random Energy Model) de Derrida na presença de um campo aleatório. Além da interação de $p$ spins, consideramos a presença de interações uniformes ferro ou antiferromagnéticas de alcance infinito.

Tanto no caso ferro quanto antiferromagnético, empregamos dois procedimentos para tratar o problema: o método de réplicas no ensemble canônico e o método da contagem de estados no ensemble microcanônico. No método de réplicas resolvemos o problema para qualquer valor de $p$ tanto sem quebra da simetria de permutação entre réplicas, quanto com um passo de quebra de simetria de Parisi. Deste modo, recuperamos resultados conhecidos para alguns modelos já estudados na literatura. Em seguida, tomamos o limite $p \rightarrow \infty$ que fornece uma solução completa para o problema do REM na presença de um campo aleatório. No método da contagem de estados, aplicável apenas no limite $p \rightarrow \infty$, mostramos que podemos estender a solução de Derrida mesmo na presença de um campo aleatório. Isso nos permitiu fazer a contagem de estados evitando assim o problema da "catástrofe da entropia negativa" presente na solução réplica simétrica. Além disso, mostramos que qualquer sistema que seja solúvel sem a interação aleatória de $p$ spins continua solúvel na presença dessa interação no limite $p \rightarrow \infty$. Portanto, concluímos que a interação aleatória de $p$ spins é somente adicionar um carácter vidro de spin ao sistema.

Obtivemos expressões gerais válidas para qualquer distribuição do campo aleatório, embora a análise numérica tenha sido restrita às distribuições duplo-delta e gaussiana. 
Estudamos a influência do campo aleatório sobre os diagramas de fases e, em particular, mostramos que podem surgir pontos tricríticos no caso de uma distribuição duplo-delta. 


\section{Abstract}

We studied the effect of a random field on spin-glass models with infinite-ranged $p$ spin interactions with a Gaussian probability distribution. The case $p=2$ corresponds to the Sherrington-Kirkpatrick model in the presence of a random field. The case $p \rightarrow \infty$ corresponds to the REM (Random Energy Model) introduced by Derrida in the presence of a random field. Besides the $p$-spin interactions we also included uniform infinite-ranged ferromagnetic and antiferromagnetic interactions.

Both in the case of ferromagnetic and antiferromagnetic interactions we employed two different approaches: The replica method in the canonical ensemble and the method of counting of the states in the microcanonical ensemble. In the replica method we solved the problem for arbitrary $p$ both in the case of replica symmetry and in the first step of Parisi's replica-symmetry breaking scheme. This allowed us to rederive results for some models already known in the lirature. Next we took the limit $p \rightarrow \infty$ which yielded a complete solution to the REM in a random field. In the method of counting of the states, which is effective only in the limit $p \rightarrow \infty$, we showed that we can extend the Derrida's solution even in the presence of a random field. This allowed us to do the counting of the states avoiding the so called negative-entropy catastrophe present in the replica-symmetric solution. We also showed that any solvable model without random $p$ spin interactions is also solvable in the presence of such interactions in the limit $p \rightarrow \infty$. Therefore, we conclude that the $p$-spin random interactions only add a spin-glass character to the system.

We have obtained general expressions valid for any random-field distributions, although we limited the numerical analysis to double-delta and Gaussian distributions. We studied 
the effects of the random field on the phase diagrams, and in particular, we showed the possibility of tricritical point in the case of double-delta distributions. 


\section{Sumário}

1 Introdução $\quad 1$

2 O modelo de $p$ spins na presença de um campo aleatório 13

2.1 O Modelo . . . . . . . . . . . . . . . . . . . . . 13

2.2 Solução Réplica Simétrica . . . . . . . . . . . . . . . . . . 20

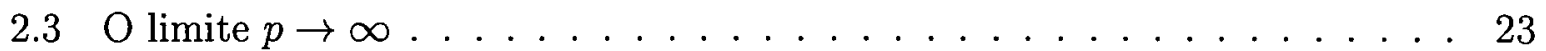

2.4 Estabilidade da solução com simetria entre réplicas . . . . . . . . . . . . 23

2.4.1 Os autovetores longitudinais . . . . . . . . . . . 25

2.4 .2 Os autovetores anômalos . . . . . . . . . . . . 25

2.4 .3 Os autovetores transversais $\ldots \ldots \ldots \ldots$

2.4 .4 Condição de estabilidade . . . . . . . . . . . . . . . . 27

2.5 Quebra de Simetria entre Réplicas . . . . . . . . . . . . . . . . 28

2.6 Caso $J_{0}=0$ : ausência de interação ferromagnética entre pares . . . . . . 32

2.6 .1 Distribuição duplo-delta . . . . . . . . . . . . . . 33

2.6.2 Distribuição de campo gaussiano (normal) . . . . . . . . . . 35

2.7 Caso $J_{0}>0$ : com interação ferromagnética entre pares $\ldots \ldots \ldots$. . . 36

2.7.1 Determinação do ponto tricrítico . . . . . . . . . . . . . 40

3 Modelo de energia aleatória no ensemble microcanônico 42

3.1 Relação com o modelo de energias aleatórias . . . . . . . . . . . . . . 43

3.2 Solução no ensemble microcanônico . . . . . . . . . . . . . . . . . . . . 49

3.3 Modelo com campos aleatórios . . . . . . . . . . . . . . . 52 
4 REM antiferromagnético em um campo aleatório 56

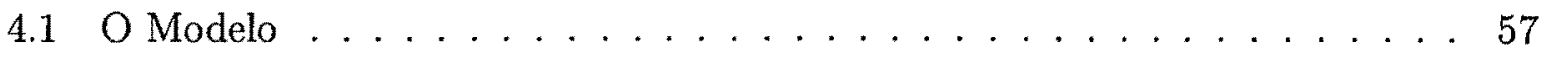

4.2 Solução Réplica Simétrica . . . . . . . . . . . . . . . . 61

4.3 Casos limites . . . . . . . . . . . . . . . . . 62

4.4 Distribuições de campo aleatório . . . . . . . . . . . . . 63

5 Solução do REM antiferromagnético no ensemble microcanônico $\quad 64$

5.1 Relação com o modelo de energias aleatórias . . . . . . . . . . . 65

5.2 Solução no ensemble microcanônico . . . . . . . . . . . . . 70

5.3 Modelo antiferromagnético em um campo aleatório . . . . . . . . . 72

$\begin{array}{lll}6 & \text { Conclusões } & 76\end{array}$

$\begin{array}{ll}\text { A Cálculo dos elementos da matriz hessiana } & 78\end{array}$

B Função de partição do REM $\quad 82$

C Cálculo alternativo da energia livre sem o uso de réplicas 89

Referências Bibliográficas $\quad 93$ 


\section{Lista de Figuras}

1.1 (a) plaqueta não frustrada e (b) plaqueta frustrada . . . . . . . . . . 6

2.1 Diagrama de campo $\times$ temperatura para uma distribuição duplo-delta com

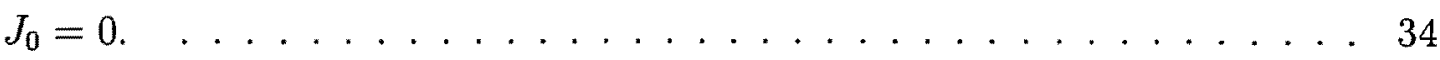

2.2 Diagrama campo $\times$ temperatura para uma distribuição normal com $J_{0}=0 \quad 35$

2.3 diagrama de fases para campo zero . . . . . . . . . . . . . 36

2.4 diagrama de fases para uma distribuição duplo-delta com $\sigma=0,2 \ldots 37$

2.5 diagrama de fases para uma distribuição gaussiana com $\sigma=0,2 \ldots 37$

2.6 diagrama de fases para uma distribuição duplo-delta com $\sigma=0,4 \ldots 38$

2.7 diagrama de fases para uma distribuição normal com $\sigma=0,4 \ldots \ldots$

2.8 diagrama de fases para uma distribuição duplo-delta com $\sigma=0,8 \ldots 39$

2.9 diagrama de fases para uma distribuição normal com $\sigma=0,8 \ldots \ldots 39$

2.10 diagrama de fases para uma distribuição de campo duplo-delta com $\sigma=1,040$ 


\section{Capítulo 1}

\section{Introdução}

Sólidos cristalinos perfeitos têm sido estudados intensamente desde o surgimento da Mecânica Quântica. Sabemos, entretanto, que os materiais existentes na natureza apresentam sempre algum tipo de imperfeição. Por muitos anos qualquer imperfeição ou desordem na estrutura regular da rede era tratada como uma perturbação fraca dos estados de Bloch, ainda que a presença dessas imperfeições pudesse alterar as propriedades do material de maneira significativa. Somente muitos anos depois o estudo dos sistemas desordenados tomou grande impulso com o artigo seminal de P. W. Anderson que investigava o comportamento quântico de um elétron em um meio sem qualquer ordem cristalina (Anderson, 1958). A partir de então, a Física dos Sistemas Desordenados passou a ser tratada como uma nova área de investigação e não mais como um adendo ao teorema de Bloch.

Durante as últimas décadas o estudo dos sistemas magnéticos com desordem tem evoluído rapidamente, tanto do ponto de vista teórico quanto experimental, contribuindo para o aumento do nosso entendimento da Física dos Sistemas Desordenados. As razões para tal desenvolvimento encontram-se tanto na simplicidade dos modelos magnéticos, que capturam os aspectos básicos de sistemas mais complicados, quanto na existência de muitas realizações experimentais de tais sistemas (Fisher, Grinstein, e Khurana, 1988).

Cabe aqui caracterizar os casos limites de desordem encontrados nos sistemas magnéticos e como estes podem ser tratados. Para tanto, é necessário distinguir claramente a desordem nos estados de spins dos átomos (variáveis dinâmicas) e a desordem espacial 
entre eles (variáveis de desordem). No caso em que as variáveis de desordem e as variáveis dinâmicas estão em equilíbrio térmico temos o limite recozido (ou annealed), enquanto no caso em que as variáveis dinâmicas e as variáveis de desordem estão termicamente desacopladas (fora de equilíbrio termodinâmico) temos o limite temperado ${ }^{1}$ (ou quenched). No caso temperado as variáveis de desordem estão fixas ou congeladas, ou seja, a distribuição dos átomos não muda com a temperatura. A distinção entre os limites temperados e recozidos baseia-se na diferença entre as escalas de tempo de relaxação das variáveis dinâmicas e de desordem. O limite recozido é apropriado para uma descrição de sistemas nos quais os tempos de relaxação das variáveis dinâmicas e de desordem são da mesma ordem do tempo de observação experimental das medidas magnéticas. No caso temperado, por sua vez, o tempo de relaxação das variáveis de desordem é muito maior que o tempo de relaxação das variáveis dinâmicas. O caso temperado corresponde mais às situações experimentais, sendo de grande importância saber como tratar, no contexto da Física Estatística, o limite de desordem temperada. A solução foi fornecida por Brout (Brout, 1959) e posteriormente reanalisada por Mazo (Mazo, 1963). Resumiremos a seguir o argumento heurístico devido a Brout.

Consideremos medidas experimentais em uma única amostra macroscópica, correspondendo a uma única realização de desordem temperada. A energia livre de Helmholtz da amostra será

$$
F_{\kappa}=-k_{B} T \ln Z_{\kappa}
$$

em que $k_{B}$ é a constante de Boltzmann e o índice $\kappa$ indica uma dada configuração para os estados de desordem do sistema. Lembramos que as realizações de desordem variam de amostra para amostra, mas as medidas experimentais são as mesmas, supondo que as amostras sejam preparadas da mesma forma. Brout argumenta que se dividirmos, mentalmente, um sistema macroscópico em um número muito grande $N$ de subsistemas (note que os subsistemas são também considerados macroscópicos) e desprezarmos as interaçōes entre os subsistemas (considerando forças de curto alcance), a função de partição pode ser

\footnotetext{
${ }^{1}$ Essa terminologia origina-se na Metalurgia. Recozer um metal significa esfriá-lo lentamente mantendo equilíbrio térmico com o ambiente, enquanto temperar um metal significa aquecê-lo a altas temperaturas e resfriá-lo rapidamente de modo a manter o metal no estado de altas temperaturas (possivelmente metaestável).
} 
fatorada como um produto de funções de partição dos subsistemas. Portanto, a energia livre de Helmholtz será dada por

$$
F_{\kappa}=-k_{B} T \ln Z_{\kappa}=-k_{B} T \sum_{i=1}^{N} \ln Z_{\kappa}^{(i)}=\sum_{i=1}^{N} F_{\kappa}^{(i)}
$$

em que o índice $i$ indica o $i$-ésimo subsistema. Como esses subsistemas são independentes, as energias livres de Helmholtz $F_{\kappa}^{(i)}$ são variáveis aleatórias independentes. De acordo com o teorema central do limite (James, 1981), no limite $N \rightarrow \infty$ as energias livres $F_{\kappa}$ estarão distribuídas normalmente em torno do valor esperado com desvio padrão proporcional a $\sqrt{N}$. Como a flutuação é desprezível, temos

$$
F_{\kappa}=\left\langle F_{\kappa}\right\rangle_{\kappa}=-k_{B} T\left\langle\ln Z_{\kappa}\right\rangle_{\kappa}
$$

ou seja, é como se o sistema fizesse ele próprio a média sobre a desordem espacial. Essa propriedade é chamada self-averaging em inglês.

O argumento de Brout foi posteriormente formalizado por Mazo sem precisar supor que as interações são de curto alcance, mas apenas que as interações são aleatórias segundo uma distribuição de probabilidade a priori. Consideremos um sistema que possa assumir somente uma quantidade enumerável de configurações. $\mathrm{O}$ índice $\kappa$ especifica as variáveis de desordem, enquanto o índice $s$ refere-se às variáveis dinâmicas. O estado de um sistema $(\kappa, s)$ em equilíbrio tem uma probabilidade de ocorrência dada por

$$
P(\kappa, s)=p(\kappa) G(s \mid \kappa)
$$

em que $p(\kappa)$ é a probabilidade dada a priori e é independente dos estados dinâmicos do sistema (caso temperado) e $G(s \mid \kappa)$ é uma probabilidade condicional do estado dinâmico $s$ em dado $\kappa$. A entropia do sistema pode ser escrita como

$$
S=-k_{B} \sum_{\{\kappa, s\}} P(\kappa, s) \ln P(\kappa, s)
$$

e deve ser maximizada sujeita aos seguintes vínculos:

$$
\sum_{\{\kappa, s\}} E_{\kappa, s} P(\kappa, s)=E
$$

sendo a energia interna $E$ uma grandeza que é mantida fixa e

$$
\sum_{\{s\}} G(s \mid \kappa)=1
$$


para todo $\kappa$ no qual $p(\kappa)>0$. O máximo restrito da entropia é encontrado maximizando a função

$$
\sum_{\{\kappa, s\}}\left\{-k_{B} p(\kappa) G(s \mid \kappa) \ln [p(\kappa) G(s \mid \kappa)]-\beta p(\kappa) G(s \mid \kappa) E_{\kappa, s}+\alpha_{\kappa} G(s \mid \kappa)\right\}
$$

em que $\alpha_{\kappa}$ e $\beta$ são os multiplicadores de Lagrange. Variando $G(s \mid \kappa)$ e impondo os vínculos (1.6) e (1.7), obtemos

$$
G(s \mid \kappa)=\frac{e^{-\beta E_{\kappa, s}}}{\sum_{\{s\}} e^{-\beta E_{\kappa, s}}}
$$

com o denominador identificado como a função de partição do sistema para uma realização particular $\kappa$ das variáveis aleatórias e o fator $\beta$ é associado da maneira usual como o inverso da temperatura $\operatorname{com} \beta=1 / k_{B} T$.

A entropia do sistema fica

$$
S=\frac{E}{T}-k_{B} \sum_{\{\kappa\}} p(\kappa) \ln p(\kappa)+k_{B} \sum_{\{\kappa\}} p(\kappa) \ln Q(\kappa)
$$

$\operatorname{com} Q(\kappa)=\sum_{\{s\}} e^{-\beta E_{\kappa, s}}$. A energia livre de Helmholtz será dada por

$$
F=E-T S=-\beta^{-1}\left[\sum_{\{\kappa\}} p(\kappa) \ln Q(\kappa)-\sum_{\{\kappa\}} p(\kappa) \ln p(\kappa)\right]
$$

em que o último termo representa a entropia de mistura devido à desordem. Mazo argumenta que esse termo é irrelevante, pois qualquer medida da entropia está associada a sua variação e a distribuição de probabilidades $p(\kappa)$ da desordem é fixa.

Existem inúmeros exemplos de sistemas magnéticos desordenados, mas vidros de spin e ferromagnetos em campos aleatórios têm desempenhado papel central no desenvolvimento das teorias mais recentes (Young, 1997). Tanto o vidro de spin quanto o campo aleatório podem ser obtidos experimentalmente diluindo-se sistemas magnéticos puros. Para que a diluição dê origem a um vidro de spin é necessário que haja interações competitivas entre os momentos magnéticos no sistema puro. Para que a diluição simule o efeito de um campo aleatório é preciso que o sistema puro seja antiferromagnético e que um campo uniforme seja aplicado.

Apesar de intensos esforços, muitas questões de relevância em vidros de spin e campos aleatórios ainda permanecem em aberto, não havendo consenso sobre a descrição teórica 
mais apropriada para os vidros de spin reais. Contudo, as novas técnicas e conceitos desenvolvidos ao longo dos estudos destes sistemas encontraram vastas aplicações em outros campos das Ciências, como no estudo de problemas de optimização combinatória (Mézard, Parisi, e Virasoro, 1987), partição de números, na modelagem de redes neurais (Amit, 1989), enovelamento de proteínas e, mais recentemente, no estudo de modelos para evolução biológica, entre outros. Isso tem permitido uma conexão muito ampla entre a Física Estatística e as Matemáticas Pura e Aplicada, a Computação e a Biologia.

Os vidros de spin são estudados há algumas décadas (Binder e Young, 1986; Fischer e Hertz, 1991; Mydosh, 1993; Dotsenko, 1994; Mézard et al., 1987). Os vidros de spin canônicos são as ligas metálicas diluídas do tipo AuFe, AuMn, CuMn e AgCr, em que uma pequena fração (1 a $10 \%$ ) de íons magnéticos (Fe, Mn, Cr) estão espalhados aleatoriamente numa matriz metálica não-magnética $(\mathrm{Au}, \mathrm{Cu}, \mathrm{Ag})$. Um marco na área de vidros de spin ocorreu com as experiências de Cannella e Mydosh (Cannella e Mydosh, 1972). Eles observaram que a suscetibilidade magnética em ligas metálicas do tipo CuMn e AuFe apresentam uma cúspide acentuada numa certa temperatura, sugerindo a ocorrência de uma transição de fases para uma fase vítrea. Trabalhos experimentais posteriores revelaram diversos fenômenos que não se coadunam com as transições de fases tradicionais. Além do mais, tornou-se claro que esses fenômenos não se limitam apenas a ligas diluídas de ions magnéticos em metais nobres, mas que são comuns a muitos materiais magnéticos, como em compostos de terras raras, e inclusive não magnéticos, como os ferroelétricos desordenados de $\mathrm{KTaO}_{3}$ diluídos com Li (Höchli, 1982).

Tentativas teóricas de compreensão dos vidros de spin iniciaram-se com o modelo proposto por Edwards e Anderson (EA). Estes autores utilizaram o método de réplicas para calcular o logaritmo da função de partição e introduziram o parâmetro de ordem $\left(q_{E A}\right)$ para caracterizar a fase vidro de spin,

$$
q_{E A}=\frac{1}{N} \sum_{i=1}^{N}\left\langle\left\langle S_{i}^{2}\right\rangle_{T}\right\rangle_{J}
$$

em que $\langle\cdots\rangle_{T}$ e $\langle\cdots\rangle_{J}$ representam as médias térmicas e sobre a desordem respectivamente (Edwards e Anderson, 1975). Posteriormente, estabeleceram-se os ingredientes necessários para o surgimento da fase vidros de spin: frustração e desordem (Toulouse, 1977). A 
frustração é a impossibilidade de satisfazer todas as ligações simultaneamente em um circuito fechado da rede chamado plaqueta, ou de outro modo, a frustração pode ser vista como a competição entre as diferentes interações atuando em um certo momento magnético. Na figura (1.1) mostramos duas plaquetas com ligações aleatórias. Em 1977,

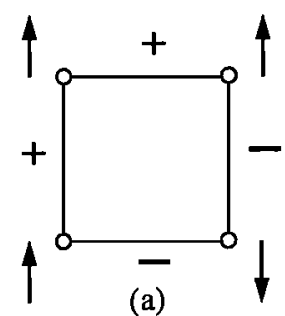

(a)

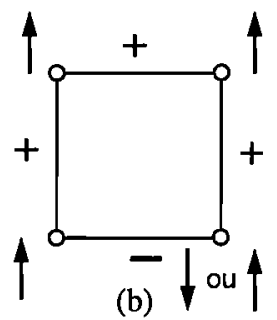

(b)

Figura 1.1: (a) plaqueta não frustrada e (b) plaqueta frustrada

Toulouse introduziu a função $\Phi=\prod_{(c)} \operatorname{sinal}\left(J_{i j}\right)$, que mede o efeito da frustração em uma plaqueta $c$ (Toulouse, 1977). Se $\Phi=1$, a plaqueta não é frustrada, e se $\Phi=-1$ a plaqueta é dita frustrada. Deste modo, pode-se verificar que não é qualquer desordem que induz frustração. A desordem ou aleatoriedade nas posições dos íons magnéticos constitui outro elemento necessário para a obtenção do comportamento vidro de spin. Nas ligas metálicas (como AuFe) os ions magnéticos estão muitos diluídos, mas existem interações entre eles mediadas por elétrons de condução dos íons não magnéticos. Esta interação de troca indireta, chamada de interação RKKY (Ruderman e Kittel, e Kasuya e Yosida) (Ashcroft e Mermin, 1976), apresenta um comportamento oscilatório com a distância entre os íons magnéticos, podendo ser tanto positiva (ferromagnética), favorecendo o alinhamento paralelo, quanto negativa (antiferromagnética), favorecendo o alinhamento antiparalelo dos íons. Como a distância $r$ entre os íons magnéticos é aleatória, a interação entre eles também será aleatória. A interação RKKY oscila com período comparável ao espaçamento da rede e sua intensidade decai com $r^{-3}$. No modelo EA essas interações são supostas de curto alcance e distribuídas de forma gaussiana (ou normal).

Logo em seguida ao trabalho de Edwards e Anderson, Sherrington e Kirkpatrick (SK) introduziram um modelo de vidros de spin com spins tipo Ising e interações de alcance infinito obedecendo a uma distribuição gaussiana ou normal (Sherrington e Kirkpatrick, 
1975). Neste modelo a hamiltoniana é dada por

$$
\mathcal{H}=\sum_{1 \leq i<j \leq N} J_{i j} S_{i} S_{j},
$$

em que $J_{i j}$ tem média zero e variância $J^{2} / N$ ou em notação usual $J_{i j} \sim N\left(0, J^{2} / N\right)$. A densidade de probabilidade é dada por

$$
p\left(J_{i j}\right)=\sqrt{\frac{N}{2 \pi J^{2}}} \exp \left(-\frac{J_{i j}^{2} N}{2 J^{2}}\right) .
$$

A solução do modelo SK obtida por Sherrington e Kirkpatrick concordava qualitativamente com diversos comportamentos observados em vidros de spin reais. Entretanto, apresentou problemas em baixas temperaturas, pois a entropia tornava-se negativa, o que é impossível num sistema com um número contável de estados acessíveis. Anderson denominou esse comportamento esdrúxulo de "catástrofe da entropia negativa" (Anderson, 1988).

Em 1978, Almeida e Thouless (AT) identificaram o problema na solução proposta por Sherrington e Kirkpatrick (Almeida e Thouless, 1978). Essa solução, hoje denominada solução com simetria entre réplicas, é instável em toda fase de vidro de spin, isto é, não corresponde a um extremo bem definido na aplicação do método de Laplace. Na presença de um campo magnético externo, a chamada linha de transição AT separa a região de altas temperaturas, onde a solução com simetria entre réplicas é estável, da região de baixas temperaturas onde ela é instável. A origem dessa instabilidade estava na suposição da solução apresentar simetria por permutação do índice das réplicas. A sugestão desses autores para resolver o problema era quebrar a simetria entre as réplicas.

Após tentativas de vários pesquisadores, Parisi encontrou uma solução estável para o modelo SK na região de baixas temperaturas, denominada solução com quebra de simetria entre réplicas (Parisi, 1979, 1980b, 1980a). Essa solução requer a introdução de uma função parâmetro de ordem contínua, isto é, um número infinito de parâmetros de ordem associada a uma multiplicidade de estados em baixas temperaturas.

Até o presente, a validade da solução do modelo SK para sistemas reais (interação de curto alcance) permanece uma questão polêmica, existindo ainda grande controvérsia no que diz respeito à aplicabilidade das propriedades previstas pela teoria de campo médio 
dos vidros de spin. Como alternativa, foi proposto por Fisher o modelo de gotas (ou droplet model), baseado em argumentos de grupo de renormalização sobre paredes de domínio (Fisher e Huse, 1986). Segundo esse modelo, os vidros de spin com interação de curto alcance devem apresentar, na região de baixas temperaturas, apenas dois estados fundamentais, relacionados entre si por simetria por inversão como ocorre em sistemas ferromagnéticos. O modelo de gotas torna-se questionável ao aumentarmos a dimensionalidade do sistema, quando se espera um comportamento do tipo campo médio acima de uma dimensão crítica $d_{l}$. Acredita-se que para vidros de spin a dimensão crítica acima da qual a teoria de campo médio esteja correta seja $d_{l}=6$ (Dominicis, Kondor, e Temesvari, 1993).

Paralelamente aos vidros de spin, os sistemas com campo aleatório têm sido estudados intensamente há várias décadas (Shapir, 1992). Entretanto, restam muitas questões ainda não respondidas (Gofman, Adler, Aharony, Harris, e Schwartz, 1996; Newman e Barkema, 1996), com exceção da prova rigorosa do argumento de Imry-Ma (Imry e Ma, 1975), que estabelece a existência de ordem de longo alcance em três ou mais dimensões em temperatura $T=0$ (Imbrie, 1984) e em temperaturas suficientemente baixas (Bricmont e Kupiainen, 1987). Esses argumentos que estabelecem a dimensão crítica inferior $d_{l}=2$, tratam apenas da competição entre as energias de troca e a energia do campo aleatório, diferente, portanto, da batalha energia-entropia que controla o papel das transições de fase nos sistemas puros e sem frustração. Aqui as flutuações térmicas têm um papel secundário, sendo o efeito de desordenar o sistema preponderantemente devido ao campo aleatório.

Uma questão importante consiste em determinar a relação entre o problema de campos aleatórios e os vidros de spin (Almeida e Bruinsma, 1987; Mézard e Young, 1992; Mézard e Monasson, 1994). Campos aleatórios não podem ser gerados diretamente no laboratório. Contudo, Fishman e Aharony (Fishman e Aharony, 1979) e Cardy (Cardy, 1984) mostraram que antiferromagnetos diluídos, na presença de um campo uniforme, fornecem realizações experimentais desses sistemas (Belanger, 1997), e que as propriedades críticas (estáticas) desses dois sistemas podem ser as mesmas. Um exemplo de realização 
experimental do modelo de Ising bidimensional na presença de um campo aleatório é o sistema diluído $\mathrm{Rb}_{2} \mathrm{Co}_{0.85} \mathrm{Mg}_{0.15} \mathrm{~F}_{4}$ (Ferreira, King, Jaccarino, e Cardy, 1983). Note que, assim como os vidros de spin, os sistemas com campos aleatórios também apresentam frustração, resultante da competição entre as interações de troca que favorecem o alinhamento paralelo dos spins, e o campo aleatório local que tende a orientar os spins segundo sua direção.

Embora os problemas de vidros de spin e de campos aleatórios sejam em geral considerados separadamente, há sistemas em que o mecanismo responsável pela ocorrência da fase vidro de spin gera também um campo efetivo aleatório. Por exemplo, a necessidade da inclusão tanto do campo aleatório quanto da aleatoriedade nas interações foi sugerida no caso da mistura ferro-antiferroelétrica $\mathrm{Rb}_{1-x}\left(\mathrm{NH}_{4}\right)_{x} \mathrm{H}_{2} \mathrm{PO}_{4}$ (Pirc, Tadić, e Blinc, 1987; Levstik et al., 1991; Pirc, Blinc, e Wiotte, 1992). Um outro exemplo são os vidros de spin antiferromagnéticos como $\mathrm{Fe}_{x} \mathrm{Mg}_{1-x} \mathrm{Cl}_{2}$ (Bertrand, Fert, Schmidt, Bensamka, e Legrand, 1982; Wong et al., 1985; Wong, Yoshizawa, e Shapiro, 1985), em que esperamos que seja gerado um campo aleatório pelo mecanismo usual (Fishman e Aharony, 1979; Cardy, 1984). É, portanto, interessante estudar o efeito do campo aleatório nos modelos de vidros de spin. Os efeitos do campo aleatório no modelo SK ferromagnético ( $\mathrm{Ma}$, Gong, e Li, 1991; Soares, Nobre, e Almeida, 1994; Nogueira, Nobre, Costa, e Coutinho, 1998) bem como no modelo de vidro de spin $m$-vetorial (Nogueira, Nobre, Coutinho, e Almeida, 1996) foram investigados recentemente. O modelo SK ferromagnético na presença do campo aleatório (Soares et al., 1994) parece fornecer uma descrição qualitativa da evolução dos diagramas de fase campo-temperatura do antiferromagneto diluído $\mathrm{Fe}_{x} \mathrm{Zn}_{1-x} \mathrm{~F}_{2}$. Recentemente foi estudado o efeito de campo aleatório num modelo SK de duas sub-redes, apropriado à descrição de vidros de spin antiferromagnéticos (Vieira, Nobre, e Yokoi, 2000). Todos esses estudos limitam-se à consideração da solução com simetria entre réplicas. Mas, como vimos, é justamente na região de baixas temperaturas que a solução réplica simétrica apresenta problemas. Embora seja importante investigar a solução com quebra da simetria entre réplicas, o estudo numérico das equações resultantes não é simples. Assim, seria muito interessante dispor de um modelo no qual fosse possível 
investigar o efeito da quebra da simetria entre réplicas sem que fossem necessários cálculos numéricos proibitivos.

Um possível candidato é o modelo de energias aleatórias ou REM (Random Energy Model em inglês) (Derrida, 1980). Este modelo pode ser visto como o caso limite de uma família de modelos que generalizam o modelo SK, permitindo interações aleatórias de $p$ spins. A hamiltoniana para este sistema é

$$
\mathcal{H}=\sum_{1 \leq i_{1}<\cdots i_{p} \leq N} J_{i_{1} \cdots i_{p}} S_{i_{1}} \cdots S_{i_{p}}
$$

com $J_{i_{1} \cdots i_{p}} \sim N\left(0, J^{2} p ! / 2 N^{p-1}\right)$. A densidade de probabilidade é

$$
p\left(J_{i_{1} \cdots i_{p}}\right)=\sqrt{\frac{N^{p-1}}{p ! \pi J^{2}}} \exp \left(-\frac{J_{i_{1} \cdots i_{p}}^{2} N^{p-1}}{p ! J^{2}}\right) .
$$

No caso $p=2$ recuperamos o modelo SK, enquanto o limite $p \rightarrow \infty$ corresponde ao modelo de energias aleatórias. Do ponto de vista teórico, o que torna este modelo interessante é o fato de ser exatamente solúvel com ou sem o uso do método de réplicas. O REM foi chamado de "o modelo mais simples de vidro de spin" (Gross e Mézard, 1984). No caso geral com $p>0$ o modelo foi resolvido por Gross e Mézard (Gross e Mézard, 1984) usando o formalismo de réplicas, sendo reanalisado posteriormente por Gardner (Gardner, 1985). Esses trabalhos mostraram que para valores grandes de $p$ a função parâmetro de ordem tem um comportamento análogo ao vidro de spin de Potts (Gross, Kanter, e Sompolinsky, 1985): logo abaixo da transição a função $q(x)$ torna-se uma função degrau, passando a ter um comportamento similar ao modelo SK (monótona e crescente em $x$ ) em baixas temperaturas. Apesar deste tipo de modelo ter sido introduzido como um problema puramente teórico, seu estudo recebeu atenção considerável devido à identificação de analogias entre suas transições estáticas e dinâmicas e as respectivas transições obtidas em teorias de vidros estruturais (Kirkpatrick e Thirumalai, 1987b, 1987a). Outros estudos recentes foram realizados levando em conta efeitos de inclusão de campos magnéticos uniformes (Oliveira e Fontanari, 1999; Gillin e Sherrington, 2001), de interações ferro (Dorlas e Wedagedera, 1999; Gillin e Sherrington, 2000; Gillin, Nishimori, e Sherrington, 2001) e antiferromagnéticas (Almeida, 1998, 1999) e de competições entre mais de um tipo de desordem (Queiroz Jr., Costa, e Nobre, 2002). 
Nesta tese investigamos os efeitos de campos aleatórios num REM de uma ou duas sub-redes, considerando o problema com ou sem o emprego do método de réplicas. O procedimento padrão do método de réplicas é feito no ensemble canônico, onde encontramos a energia livre por meio da função de partição do sistema. Embora tal escolha pareça ser a mais natural, temos que lançar mão de um esquema bastante sofisticado (quebra de simetria entre réplicas) a fim de obtermos uma solução correta para o modelo. Uma outra maneira de tratar o problema seria tentar resolvê-lo no ensemble microcanônico, fazendo uma contagem dos estados, de modo a garantir que não tenhamos o problema da "catástrofe da entropia negativa". Contudo, o ensemble microcanônico só é viável em condições muito particulares. Especificamente, dentro da classe dos modelos com interações de $p$ spins, somente no limite $p \rightarrow \infty$ é possível resolver o problema exatamente. Nos capitulos que se seguem investigamos as duas abordagens.

No capítulo 2 investigamos o modelo com interações aleatórias de $p$ spins na presença de um campo aleatório pelo método de réplicas. Inicialmente determinamos a solução com simetria entre réplicas (incorreta na região de baixas temperaturas por apresentar entropia negativa) para qualquer valor finito de $p$. A seguir estudamos a solução de Parisi com um passo de quebra de simetria entre réplicas.

No capítulo 3 tratamos o mesmo problema do capítulo 2 , no limite $p \rightarrow \infty$, no ensemble microcanônico, conforme a abordagem inicial de Derrida para o modelo de energias aleatórias. Estendemos a solução de Derrida na presença de um campo aleatório. Mostramos além disso que qualquer sistema que seja solúvel sem a interação aleatória de $p$ spins continua solúvel com a presença dessa interação. A presença da interação aleatória de $p$ spins é simplesmente acrescentar um caráter vidro de spin ao sistema.

No capítulo 4 investigamos um sistema com interações aleatórias de multispins e interações antiferromagnéticas entre sub-redes na presença de um campo aleatório. Aqui novamente utilizamos o método de réplicas e encontramos a solução para toda uma família de modelos vidros de spin antiferromagnéticos recuperando o funcional energia livre para alguns modelos conhecidos na literatura.

No capítulo 5 apresentamos a solução do modelo antiferromagnético dentro do ensemble 
microcanônico sem o uso do método de réplicas.

Finalmente, no capítulo 6 apresentamos as conclusões do presente trabalho. 


\section{Capítulo 2}

\section{O modelo de $p$ spins na presença de}

\section{um campo aleatório}

Neste capítulo apresentamos a inclusão de um campo aleatório no modelo de vidro de spin com interação de $p$ spins. Determinamos o funcional energia livre, pelo método de réplicas, para qualquer valor de $p$ de modo a obter toda uma classe de modelos que generalizam o modelo SK. Estaremos interessado no limite $p \rightarrow \infty$ (modelo de energias aleatórias), em que ocorre uma simplificação e nos permite determinar o diagrama de fases correto do modelo sem cálculos numéricos proibitivos.

\subsection{O Modelo}

Consideremos uma classe de modelos definida pela hamiltoniana,

$$
\mathcal{H}=-\sum_{1 \leq i_{1}<\cdots<i_{p} \leq N} J_{i_{1} \cdots i_{p}} S_{i_{1}} \cdots S_{i_{p}}-\frac{J_{0}}{N} \sum_{1 \leq i<j \leq N} S_{i} S_{j}-\sum_{i=1}^{N} H_{i} S_{i},
$$

em que $S_{i}= \pm 1$ são spins de Ising, $\operatorname{com} i=1,2, \cdots, N$. As variáveis aleatórias temperadas $J_{i_{1} \cdots i_{p}}$ são independentes e identicamente distribuídas e satisfazem à distribuição de probabilidade normal (ou gaussiana) com média zero e variância $J^{2} p ! / 2 N^{p-1}$

$$
J_{i_{1} \cdots i_{p}} \sim N\left(0, J^{2} p ! / 2 N^{p-1}\right)
$$

O modelo é de alcance infinito e existe uma interação $J_{i_{1} \cdots i_{p}}$ para qualquer grupo de $p$ spins, ou seja, o modelo de Sherrington e Kirkpatrick (SK) (Sherrington e Kirkpatrick, 1975) é 
generalizado substituindo a interação aleatória entre pares por interações aleatórias entre $p$ spins. A distribuição de probabilidade dos $J_{i_{1} \cdots i_{p}}$ é escolhida de modo a garantir a extensividade da energia livre. O fator $p$ ! em (2.2) no permite recuperar à variância do modelo SK para $p=2$. Vamos considerar o parâmetro $J_{0}$ positivo (correspondendo a acoplamentos ferromagnéticos) e que o campo externo aleatório $H_{i}$ possa apresentar qualquer distribuição, embora nos cálculos numéricos nos restringiremos apenas às distribuições normais e duplo-delta. Podemos notar que para $p=1$ e $J_{0}=0$, na hamiltoniana (2.1), obtemos o modelo de spins livre em um campo aleatório, enquanto para $p=2$ a hamiltoniana recupera o modelo SK na presença de um campo aleatório (Soares et al., 1994; Nogueira et al., 1998; Araújo, Costa, e Nobre, 2000). No caso limite $p \rightarrow \infty$ reduz-se ao modelo de energias aleatórias ou REM (Random Energy Model) introduzido por Derrida (Derrida, 1980) na presença de um campo aleatório.

A diferença entre todos esses modelos pode ser vista em termos da distribuição de probabilidade da energia $E_{S}$, para uma dada configuração de spins $S=\left(S_{1}, \cdots, S_{N}\right)$ quando $J_{i_{1} \cdots i_{p}}$ são escolhida aleatoriamente. No limite $p \rightarrow \infty$ ocorre uma simplificação que torna as energias descorrelacionada, permitindo resolver o modelo exatamente sem uso do método de réplicas, ao menos no caso de um campo magnético uniforme, conforme o procedimento introduzido por Derrida (Derrida, 1980). Veremos, no capítulo 3, como estender esse método para introduzir um termo de campo aleatório.

Cabe mencionar que na abordagem de campo médio a estrutura espacial, é completamente ignorada, uma vez que a dimensionalidade e o tipo de rede não são levados em conta para a determinação das propriedades físicas do sistema. Entretanto, podemos citar algumas virtudes do tratamento de campo médio: sua simplicidade (normalmente o primeiro passo para o entendimento das fases de um sistema), o fato do modelo ser solúvel exatamente e apresentar um comportamento não trivial (para vidros de spin mesmo o tcm tem provado ser bastante sutil), além de suas predições concordarem qualitativamente com os experimentos.

Conforme vimos no capítulo anterior a dificuldade de tratarmos sistemas com desordem temperada, em que os valores de $J_{i_{1} \cdots i_{p}}$ são congelados, advém de termos que calcular a 
média da energia livre, ou seja, determinar a média do logaritmo da função de partição

$$
f=-\lim _{N \rightarrow \infty} \frac{1}{\beta N}\left\langle\langle\ln Z\rangle_{J}\right\rangle_{H}
$$

com a função de partição dada por

$$
Z=\operatorname{Tr} e^{-\beta \mathcal{H}}
$$

sendo que o traço representa a soma múltipla

$$
\operatorname{Tr} \equiv \sum_{S_{1}=-S}^{S} \cdots \sum_{S_{N}=-S}^{S}=\prod_{i=1}^{N} \sum_{S_{i}=-S}^{S}
$$

$\langle\cdots\rangle_{J}$ e $\langle\cdots\rangle_{H}$ indicam médias com relação à distribuição de desordem das ligações de troca e dos campos aleatórios, respectivamente. Visto que as interações de troca e os campos são variáveis aleatórias independentes, suas médias podem ser feitas independentemente,

$$
\left\langle\langle\cdots\rangle_{J}\right\rangle_{H} \equiv \int_{-\infty}^{\infty} \prod_{1 \leq i_{1}<\cdots<i_{p} \leq N} d J_{i_{1} \cdots i_{p}} \wp\left(J_{i_{1} \cdots i_{p}}\right) \prod_{i=1}^{N} d H_{i} \wp\left(H_{i}\right)(\cdots) .
$$

Para contornar o problema de calcular a média do logaritmo vamos lançar mão de um artifício ${ }^{1}$ (Edwards e Anderson, 1975) que ficou conhecido pelo nome de método de réplicas e é baseado no uso da identidade

$$
\langle\ln Z\rangle_{J}=\lim _{n \rightarrow 0} \frac{\left\langle Z^{n}\right\rangle_{J}-1}{n}=\lim _{n \rightarrow 0} \frac{\ln \left\langle Z^{n}\right\rangle_{J}}{n} .
$$

Com isso, evitamos realizar uma média sobre logaritmo da função de partição $Z$, e passamos a fazer uma média sobre a desordem da função de partição de $n$ cópias idênticas e não interagentes $Z^{n}$, sendo o número de réplicas $n$ um inteiro. Isso equivale a reduzir o problema ao caso recozido que é mais tratável do ponto de vista matemático. O ponto chave do método é efetuar a média sobre a desordem antes do traço. A média assim obtida deve ser "continuada analiticamente" procedendo com o limite $n \rightarrow 0$ na tentativa de recuperarmos a solução para o sistema temperado.

Portanto, o problema de tomar a média sobre a energia livre fica reduzido ao cálculo

\footnotetext{
${ }^{1} \mathrm{O}$ primeiro a utilizar o método de réplica em Física foi Mark Kac (Kac, 1968) e posteriormente o método foi redescoberto por EA no contexto de vidros de spin.
} 
da média sobre a desordem de $Z^{n}$, para $n$ inteiro,

$$
\begin{aligned}
\left\langle Z^{n}\right\rangle_{J}= & \left\langle\prod_{\alpha=1}^{n} Z^{\alpha}\right\rangle_{J}=\operatorname{Tr} \exp \left[\beta \sum_{\alpha=1}^{n}\left(\frac{J_{0}}{N} \sum_{i<j} S_{i}^{\alpha} S_{j}^{\alpha}+\sum_{i=1}^{N} H_{i} S_{i}^{\alpha}\right)\right] \\
& \prod_{i_{1}<\cdots<i_{p}}\left\langle\exp \left(\beta J_{i_{1} \cdots i_{p}} \sum_{\alpha=1}^{n} S_{i_{1}}^{\alpha} \cdots S_{i_{p}}^{\alpha}\right)\right\rangle_{J}
\end{aligned}
$$

$\mathrm{O}$ índice $\alpha$ indica a réplica e o traço que aparece na equação (2.8) indica uma soma sobre todos os spins das $n$ réplicas,

$$
\operatorname{Tr} \equiv \prod_{\alpha=1}^{n} \prod_{i=1}^{N} \sum_{S_{i}^{\alpha}=-S}^{S}
$$

Para calcular a média sobre a distribuição de desordem $J_{i_{1} \cdots i_{p}}$ no último termo da equação (2.8), vamos utilizar o seguinte resultado: seja $X$ uma variável aleatória com média $\mu$ e variância $\sigma^{2}$,

$$
X \sim N\left(\mu, \sigma^{2}\right)
$$

Então,

$$
\langle\exp (a x)\rangle_{X}=\exp \left(a \mu+\frac{a^{2} \sigma^{2}}{2}\right)
$$

Assim, a média sobre a desordem da função de partição de $n$ cópias do sistema será

$$
\left\langle Z^{n}\right\rangle_{J}=\operatorname{Tr} \exp \left[\beta \sum_{\alpha=1}^{n}\left(\frac{J_{0}}{N} \sum_{i<j} S_{i}^{\alpha} S_{j}^{\alpha}+\sum_{i=1}^{N} H_{i} S_{i}^{\alpha}\right)+\frac{p !(\beta J)^{2}}{4 N^{p-1}} \sum_{\alpha, \beta} \sum_{i_{1}<\cdots<i_{p}} S_{i_{1}}^{\alpha} S_{i_{1}}^{\beta} \cdots S_{i_{p}}^{\alpha} S_{i_{p}}^{\beta}\right] .
$$

Observamos que, após efetuarmos a média sobre a desordem, surge um acoplamento entre as diferentes réplicas do sistema representado pelo último termo da equação (2.12). Mas com a eliminação da desordem poderemos tratar, em princípio, o problema com as técnicas tradicionais da Mecânica Estatística.

Observamos que

$$
\begin{aligned}
\sum_{\alpha, \beta} \sum_{i_{1}<\cdots<i_{p}} S_{i_{1}}^{\alpha} S_{i_{1}}^{\beta} \cdots S_{i_{p}}^{\alpha} S_{i_{p}}^{\beta}= & \sum_{\alpha, \beta}\left[\frac{1}{p !}\left(\sum_{i} S_{i}^{\alpha} S_{i}^{\beta}\right)^{p}+O\left(N^{p-1}\right)\right]=\frac{2}{p !} \sum_{\alpha<\beta}\left(\sum_{i} S_{i}^{\alpha} S_{i}^{\beta}\right)^{p} \\
& +\frac{1}{p !} \sum_{\alpha}\left[\sum_{i}\left(S_{i}^{\alpha}\right)^{2}\right]^{p}+O\left(N^{p-1}\right)
\end{aligned}
$$

Até aqui não fizemos nenhuma suposição a respeito do tipo de spin do qual estamos tratando, sendo o resultado válido para qualquer valor de spin. Particularizando para o 
caso de spins de Ising $S_{i}= \pm 1$, obtemos

$$
\sum_{\alpha, \beta} \sum_{i_{1}<\cdots<i_{p}} S_{i_{1}}^{\alpha} S_{i_{1}}^{\beta} \cdots S_{i_{p}}^{\alpha} S_{i_{p}}^{\beta}=\frac{2}{p !} \sum_{\alpha<\beta}\left(\sum_{i} S_{i}^{\alpha} S_{i}^{\beta}\right)^{p}+\frac{n N^{p}}{p !}+O\left(N^{p-1}\right) .
$$

Segue de maneira análoga que

$$
\sum_{\alpha} \sum_{i<j} S_{i}^{\alpha} S_{j}^{\alpha}=\frac{1}{2} \sum_{\alpha}\left(\sum_{i} S_{i}^{\alpha}\right)^{2}-\frac{n N}{2}
$$

Portanto,

$$
\begin{aligned}
\left\langle Z^{n}\right\rangle_{J}= & \exp \left[\frac{(\beta J)^{2} N n}{4}-\frac{\beta J_{0} n}{2}\right] \operatorname{Tr} \exp \left[\frac{(\beta J)^{2} N}{2} \sum_{\alpha<\beta} q_{\alpha \beta}^{p}+\frac{\beta J_{0} N}{2} \sum_{\alpha} m_{\alpha}^{2}\right. \\
& \left.+\beta \sum_{\alpha} \sum_{i} H_{i} S_{i}^{\alpha}\right]
\end{aligned}
$$

com as variáveis $m_{\alpha}$ e $q_{\alpha \beta}$ definidas como

$$
\begin{aligned}
& m_{\alpha}=\frac{1}{N} \sum_{i} S_{i}^{\alpha}, \\
& q_{\alpha \beta}=\frac{1}{N} \sum_{i} S_{i}^{\alpha} S_{i}^{\beta} .
\end{aligned}
$$

Transformando as variáveis discretas $m_{\alpha}$ e $q_{\alpha \beta}$ em variáveis contínuas por meio da função delta de Dirac e desprezando os termos que vão a zero no limite termodinâmico $N \rightarrow \infty$, temos

$$
\begin{aligned}
\left\langle Z^{n}\right\rangle_{J}= & \exp \left[\frac{(\beta J)^{2} N n}{4}\right] \prod_{\alpha<\beta} \int_{-\infty}^{\infty} d q_{\alpha \beta} \prod_{\alpha} \int_{-\infty}^{\infty} d m_{\alpha} \exp N\left[\frac{(\beta J)^{2}}{2} \sum_{\alpha<\beta} q_{\alpha \beta}^{p}+\frac{\beta J_{0}}{2} \sum_{\alpha} m_{\alpha}^{2}\right] \\
& \times \operatorname{Tr} \exp \left(\beta \sum_{\alpha, i} H_{i} S_{i}^{\alpha}\right) \prod_{\alpha<\beta} \delta\left(q_{\alpha \beta}-\frac{1}{N} \sum_{i} S_{i}^{\alpha} S_{i}^{\beta}\right) \prod_{\alpha} \delta\left(m_{\alpha}-\frac{1}{N} \sum_{i} S_{i}^{\alpha}\right) .
\end{aligned}
$$

Utilizando as representações integrais para a função delta

$$
\begin{aligned}
\delta\left(m_{\alpha}-\frac{1}{N} \sum_{i} S_{i}^{\alpha}\right) & =\int_{-i \infty}^{i \infty} \frac{N d \xi_{\alpha}}{2 \pi i} \exp \left[-N \xi_{\alpha}\left(m_{\alpha}-\frac{1}{N} \sum_{i} S_{i}^{\alpha}\right)\right] \\
\delta\left(q_{\alpha \beta}-\frac{1}{N} \sum_{i} S_{i}^{\alpha} S_{i}^{\beta}\right) & =\int_{-i \infty}^{i \infty} \frac{N d \lambda_{\alpha \beta}}{2 \pi i} \exp \left[-N \lambda_{\alpha \beta}\left(q_{\alpha \beta}-\frac{1}{N} \sum_{i} S_{i}^{\alpha} S_{i}^{\beta}\right)\right]
\end{aligned}
$$


obtemos

$$
\begin{aligned}
\left\langle Z^{n}\right\rangle_{J}= & \exp \left(\frac{\beta^{2} J^{2} N n}{4}\right) \prod_{\alpha<\beta} \int_{-\infty}^{\infty} d q_{\alpha \beta} \prod_{\alpha} \int_{-\infty}^{\infty} d m_{\alpha} \prod \int_{-i \infty}^{i \infty} \frac{N d \xi_{\alpha}}{2 \pi i} \prod_{\alpha<\beta} \int_{-i \infty}^{i \infty} \frac{N d \lambda_{\alpha \beta}}{2 \pi i} \\
& \exp \left\{N\left[\frac{(\beta J)^{2}}{2} \sum_{\alpha<\beta} q_{\alpha \beta}^{p}+\frac{\beta J_{0}}{2} \sum_{\alpha} m_{\alpha}^{2}-\sum_{\alpha} m_{\alpha} \xi_{\alpha}-\sum_{\alpha<\beta} q_{\alpha \beta} \lambda_{\alpha \beta}\right]\right. \\
& \left.+\frac{1}{N} \sum_{i} \ln \operatorname{Tr} \exp \left(\beta \sum_{\alpha} H_{i} S_{i}^{\alpha}+\sum_{\alpha} \xi_{\alpha} S_{i}^{\alpha}+\sum_{\alpha<\beta} \lambda_{\alpha \beta} S_{i}^{\alpha} S_{i}^{\beta}\right)\right\}
\end{aligned}
$$

De acordo com a lei dos grandes números, no limite termodinâmico $N \rightarrow \infty$ temos com probabilidade 1

$$
\frac{1}{N} \sum_{i} \ln \operatorname{Tr} \exp \left(\beta \sum_{\alpha} H_{i} S_{i}^{\alpha}+\sum_{\alpha} \xi_{\alpha} S_{i}^{\alpha}+\sum_{\alpha<\beta} \lambda_{\alpha \beta} S_{i}^{\alpha} S_{i}^{\beta}\right)=\left\langle\ln \operatorname{Tr} e^{-\beta \overline{\mathcal{H}}}\right\rangle_{H},
$$

com

$$
-\beta \overline{\mathcal{H}}=\beta \sum_{\alpha} H S^{\alpha}+\sum_{\alpha} \xi_{\alpha} S^{\alpha}+\sum_{\alpha<\beta} \lambda_{\alpha \beta} S^{\alpha} S^{\beta}
$$

$\overline{\mathcal{H}}$ é uma hamiltoniana efetiva no espaço de réplicas. O traço que aparece no último termo da eq. (2.23) sobre todos os spins das réplicas pertencentes a um dado sítio,

$$
\operatorname{Tr} \equiv \prod_{\alpha=1}^{n} \sum_{S^{\alpha}=-S}^{S}
$$

Assim, segue que

$$
\begin{array}{r}
\left\langle\left\langle Z^{n}\right\rangle_{J}\right\rangle_{H}=\exp \left[\frac{(\beta J)^{2} N n}{4}\right] \prod_{\alpha<\beta} \int_{-\infty}^{\infty} d q_{\alpha \beta} \prod_{\alpha} \int_{-\infty}^{\infty} d m_{\alpha} \prod_{\alpha} \int_{-i \infty}^{i \infty} \frac{N d \xi_{\alpha}}{2 \pi i} \prod_{\alpha<\beta} \int_{-i \infty}^{i \infty} \frac{N d \lambda_{\alpha \beta}}{2 \pi i} \\
\exp N\left[\frac{(\beta J)^{2}}{2} \sum_{\alpha<\beta} q_{\alpha \beta}^{p}+\frac{\beta J_{0}}{2} \sum_{\alpha} m_{\alpha}^{2}-\sum_{\alpha} m_{\alpha} \xi_{\alpha}-\sum_{\alpha<\beta} q_{\alpha \beta} \lambda_{\alpha \beta}+\left\langle\ln \operatorname{Tr} e^{-\beta \overline{\mathcal{H}}}\right\rangle_{H}\right] .
\end{array}
$$

Finalmente podemos escrever a média sobre a desordem da função de partição de $n$ cópias do sistema sob a forma

$$
\left\langle\left\langle Z^{n}\right\rangle_{J}\right\rangle_{H}=\prod_{\alpha<\beta} \int_{-\infty}^{\infty} d q_{\alpha \beta} \prod_{\alpha} \int_{-\infty}^{\infty} d m_{\alpha} \prod_{\alpha} \int_{-i \infty}^{i \infty} d \xi_{\alpha} \prod_{\alpha<\beta} \int_{-i \infty}^{i \infty} d \lambda_{\alpha \beta} \exp \left[-N \beta f_{n}\left(m_{\alpha}, q_{\alpha \beta}, \xi_{\alpha}, \lambda_{\alpha \beta}\right)\right]
$$


em que

$$
\begin{aligned}
\beta f_{n}\left(m_{\alpha}, q_{\alpha \beta}, \xi_{\alpha}, \lambda_{\alpha \beta}\right)= & -\frac{(\beta J)^{2} n}{4}-\frac{(\beta J)^{2}}{2} \sum_{\alpha<\beta} q_{\alpha \beta}^{p}-\frac{\beta J_{0}}{2} \sum_{\alpha} m_{\alpha}^{2}+\sum_{\alpha} m_{\alpha} \xi_{\alpha}+\sum_{\alpha<\beta} q_{\alpha \beta} \lambda_{\alpha \beta} \\
& -\langle\ln \operatorname{Tr} \exp (-\beta \overline{\mathcal{H}})\rangle_{H} .
\end{aligned}
$$

Estamos supondo que o limite termodinâmico $N \rightarrow \infty$ possa ser tomado antes do limite do número de réplica ir a zero $n \rightarrow 0$. Sherrington e Kirkpatrick chegaram a sugerir que o comportamento não físico, como o fato da entropia negativa na região de baixa temperatura, estava associada com essa troca dos limites ${ }^{2}$. Porém, Van Hemmen e Palmer mostraram não haver nenhum problema com a operação de troca dos limites (Hemmen e Palmer, 1979). No limite $N \rightarrow \infty$ a integração nas variáveis $\lambda$ e $\xi$, pode ser feita pelo método do ponto de sela. As equações de ponto de sela são dadas por

$$
\begin{aligned}
m_{\alpha} & =\left\langle\frac{\operatorname{Tr} S^{\alpha} e^{-\beta \overline{\mathcal{H}}}}{\operatorname{Tr} e^{-\beta \overline{\mathcal{H}}}}\right\rangle_{H}, \\
q_{\alpha \beta} & =\left\langle\frac{\operatorname{Tr} S^{\alpha} S^{\beta} e^{-\beta \overline{\mathcal{H}}}}{\operatorname{Tr} e^{-\beta \overline{\mathcal{H}}}}\right\rangle_{H} .
\end{aligned}
$$

A integração sobre as variáveis $m$ e $q$ pode ser feita pelo método de Laplace. A condição para $f_{n}$ ser estacionária é dada por

$$
\begin{aligned}
\xi_{\alpha} & =\beta J_{0} m_{\alpha} \\
\lambda_{\alpha \beta} & =\frac{(\beta J)^{2} p}{2} q_{\alpha \beta}^{p-1} .
\end{aligned}
$$

Deste modo segue que

$$
\left\langle\left\langle Z^{n}\right\rangle_{J}\right\rangle_{H} \sim \exp \left[-N \min \beta f_{n}\left(m_{\alpha}, q_{\alpha \beta}, \xi_{\alpha}, \lambda_{\alpha \beta}\right)\right]
$$

A energia livre por spin é dada por

$$
\begin{aligned}
\beta f=\lim _{n \rightarrow 0} \frac{1}{n}\left(\beta f_{n}\right)= & -\frac{(\beta J)^{2}}{4}-\lim _{n \rightarrow 0} \frac{1}{n}\left[-\frac{(\beta J)^{2}}{2}(p-1) \sum_{\alpha<\beta} q_{\alpha \beta}^{p}-\frac{\beta J_{0}}{2} \sum_{\alpha} m_{\alpha}^{2}\right. \\
& \left.+\left\langle\ln \operatorname{Tr} e^{-\beta \overline{\mathcal{H}}}\right\rangle_{H}\right],
\end{aligned}
$$

\footnotetext{
${ }^{2}$ Segundo os próprios autores: "We speculate that this unphysical behavior has its origin in the interchange of limits $N \rightarrow \infty$ and $n \rightarrow 0$, but that the consequences are confined to low temperatures." (Sherrington e Kirkpatrick, 1975, pg. 1795).
} 
com

$$
\overline{\mathcal{H}}=-\sum_{\alpha}\left(H+J_{0} m_{\alpha}\right) S^{\alpha}-\frac{\beta J^{2}}{2} p \sum_{\alpha<\beta} q_{\alpha \beta}^{p-1} S^{\alpha} S^{\beta}
$$

A energia interna por spin é

$$
u=\frac{\partial(\beta f)}{\partial \beta}=-\frac{\beta J^{2}}{2}-\lim _{n \rightarrow 0} \frac{1}{n}\left(\beta J^{2} \sum_{\alpha<\beta} q_{\alpha \beta}^{p}+\frac{J_{0}}{2} \sum_{\alpha} m_{\alpha}^{2}+\sum_{\alpha}\left\langle H \operatorname{Tr} S^{\alpha} e^{-\beta \bar{H}}\right\rangle_{H}\right) .
$$

Enquanto a entropia por spin é dada por

$$
\begin{aligned}
\frac{s}{k_{B}}=\beta(u-f)= & -\frac{(\beta J)^{2}}{4}-\lim _{n \rightarrow 0} \frac{1}{n}\left[\frac{(\beta J)^{2}}{2} \sum_{\alpha<\beta} q_{\alpha \beta}^{p}-\beta J_{0} \sum_{\alpha} m_{\alpha}^{2}+\beta \sum_{\alpha}\left\langle H \operatorname{Tr} S^{\alpha} e^{-\beta \mathcal{H}}\right\rangle_{H}\right. \\
& \left.-\left\langle\ln \operatorname{Tr} e^{-\beta \overline{\mathcal{H}}}\right\rangle_{H}\right] .
\end{aligned}
$$

Neste ponto devemos fazer uma escolha para os parâmetros $m_{\alpha}$ e $q_{\alpha \beta}$ de modo a encontrar uma expressão para as equações de ponto de sela, substitui-la no funcional energia livre e tomar o limite $n \rightarrow 0$ e determinar a energia livre correspondente.

\subsection{Solução Réplica Simétrica}

Como todas as réplicas são equivalentes (possuem a mesma distribuição de desordem) o problema de determinar as soluções das equações de extremos fica simplificado se considerarmos que os diversos parâmetros de ordem independem do índice de réplicas (Sherrington e Kirkpatrick, 1975). Este tipo de solução é conhecido como solução réplica-simétrica (RS) e corresponde à seguinte parametrização

$$
\begin{array}{rlrl}
m_{\alpha} & =m, & \xi_{\alpha} & =\xi, \\
q_{\alpha \beta}=q, & \lambda_{\alpha \beta} & =\lambda .
\end{array}
$$

Portanto, os cálculos no Ansatz réplica simétrica, seguem facilmente

$$
\begin{aligned}
\beta f_{n}(m, q, \xi, \lambda)= & \frac{(\beta J)^{2} n}{4}-\frac{(\beta J)^{2} q^{p}}{4} n(n-1)-\frac{\beta J_{0}}{2} m^{2} n+m \xi n+q \lambda \frac{n(n-1)}{2} \\
& -\ln \langle\operatorname{Tr} \exp (-\beta \overline{\mathcal{H}})\rangle_{H} .
\end{aligned}
$$


vamos inicialmente tratar o último termo da expressão acima

$$
\begin{aligned}
\left\langle\operatorname{Tr} e^{-\beta \mathcal{H}}\right\rangle_{H}= & \operatorname{Tr} \exp \left[(\beta H+\xi) \sum_{\alpha} S^{\alpha}+\lambda \sum_{\alpha<\beta} S^{\alpha} S^{\beta}\right]=e^{-\frac{n \lambda}{2}}\left\langle\operatorname { T r } \operatorname { e x p } \left[(\beta H+\xi) \sum_{\alpha} S^{\alpha}\right.\right. \\
& \left.\left.+\frac{\lambda}{2}\left(\sum_{\alpha} S^{\alpha}\right)^{2}\right]\right\rangle_{H}
\end{aligned}
$$

Linearizando o termo quadrático quadrático na hamiltoniana por meio da identidade normal

$$
\exp \left(\frac{a^{2}}{2}\right)=\int_{-\infty}^{\infty} \frac{d x}{\sqrt{2 \pi}} e^{-x^{2} / 2} \exp (a x) \equiv\langle\exp (a x)\rangle_{x}
$$

temos

$$
\begin{aligned}
\left\langle\operatorname{Tr} e^{-\beta \mathcal{H}}\right\rangle_{H} & =e^{-\frac{n \lambda}{2}} \int_{-\infty}^{\infty} \frac{d x}{\sqrt{2 \pi}} e^{-x^{2} / 2}\left\langle[2 \cosh (\beta H+\xi+\sqrt{\lambda} x)]^{n}\right\rangle_{H} \\
& =e^{-\frac{n \lambda}{2}}\left\langle[2 \cosh (\beta H+\xi+\sqrt{\lambda} x)]^{n}\right\rangle_{H, x} .
\end{aligned}
$$

Fazendo, $\bar{H}=\beta H+\xi+\sqrt{\lambda} x$, temos

$$
\begin{aligned}
& \left\langle\operatorname{Tr} e^{-\beta \mathcal{H}}\right\rangle_{H}=e^{-\frac{n \lambda}{2}}\left\langle(2 \cosh \bar{H})^{n}\right\rangle_{H, x} \\
& =e^{-\frac{n \lambda}{2}}\left\langle 1+n \ln 2 \cosh \bar{H}+O\left(n^{2}\right)\right\rangle_{H, x}=\exp n\left[-\frac{\lambda}{2}+\langle\ln 2 \cosh \bar{H}\rangle_{H, x}\right. \\
& \left.+O\left(n^{2}\right)\right] \text {. }
\end{aligned}
$$

Portanto,

$$
\begin{aligned}
\beta f_{n}(m, q, \xi, \lambda)= & -\frac{(\beta J)^{2}}{4} n-\frac{(\beta J)^{2} q^{p}}{4} n(n-1)-\frac{\beta J_{0}}{2} n m^{2}+n m \xi+\lambda q \frac{n(n-1)}{2} \\
& -n\left[-\frac{\lambda}{2}+\langle\ln 2 \cosh \bar{H}\rangle_{H, x}\right] .
\end{aligned}
$$

De modo que a energia livre por spin com simetria entre réplicas é dada por

$$
\begin{aligned}
\beta f= & -\frac{(\beta J)^{2}}{4}-\lim _{n \rightarrow 0} \frac{1}{n}\left[\frac{(\beta J)^{2} q^{p}}{4} n(n-1)+\frac{\beta J_{0}}{2} n m^{2}-n m \xi+\lambda q \frac{n(n-1)}{2}\right. \\
& \left.+\ln \left\langle\operatorname{Tr} e^{-\beta \mathcal{H}}\right\rangle_{H}\right] .
\end{aligned}
$$

As equações de ponto de sela (RS) ficam

$$
\begin{aligned}
& \xi=\beta J_{0} m \\
& \lambda=\frac{(\beta J)^{2}}{2} p q^{p-1} .
\end{aligned}
$$




\subsection{O limite $p \rightarrow \infty$}

Como veremos no capítulo 3 , no limite $p \rightarrow \infty$ as energias tornam-se descorrelacionadas. É o chamado modelo de energias aleatórias, REM, introduzido por Derrida (Derrida, 1980). No caso do tratamento via método de réplicas ocorre uma simplificação no limite $p \rightarrow \infty$, pois $q^{p} \rightarrow 0$ para $q<1$.

Portanto, a energia livre por spin no limite $p \rightarrow \infty$ torna-se

$$
\beta f=-\frac{(\beta J)^{2}}{4}+\frac{\beta J_{0} m^{2}}{2}-\langle\ln 2 \cosh \vec{H}\rangle_{H} .
$$

A energia interna por spin fica

$$
u=-\frac{\beta J^{2}}{2}-\frac{J_{0} m^{2}}{2}-\langle H \tanh \bar{H}\rangle_{H}
$$

A entropia por spin é

$$
\frac{s}{k_{B}}=-\frac{(\beta J)^{2}}{4}-\beta J_{0} m^{2}+\langle\ln 2 \cosh \bar{H}\rangle_{H}-\beta\langle H \tanh \bar{H}\rangle_{H}
$$

em que os parâmetros $m$ e $q$ são dados por

$$
\begin{aligned}
m & =\langle\tanh \bar{H}\rangle_{H^{\prime}} \\
q & =\left\langle\tanh ^{2} \bar{H}\right\rangle_{H}
\end{aligned}
$$

com

$$
\bar{H}=\beta\left(H+J_{0} m\right)
$$

\subsection{Estabilidade da solução com simetria entre réplicas}

A estabilidade da solução réplica simétrica pode ser determinada por meio do cálculo da derivada segunda da energia livre com respeito aos parâmetros de ordem $m_{\alpha}, q_{\alpha \beta}$. Para o modelo SK a análise de estabilidade da solução réplica simétrica foi feita por Almeida e Thouless que mostraram que a solução era instável em toda a fase vidro de spin (Almeida e Thouless, 1978). Mais que isso, mostraram que na presença de um campo uniforme essa solução era instável abaixo de uma linha hoje denominada linha de instabilidade de Almeida-Thouless (AT). Nesta seção apresentaremos a análise de estabilidade réplica simétrica do modelo de $p$ spins num campo aleatório. 
Consideremos a expansão de $f_{n}\left(m_{\alpha}, q_{\alpha \beta}\right)$ numa série de Taylor em torno da solução réplica simétrica

$$
m_{\alpha}=m+\epsilon_{\alpha} ; \quad q_{\alpha \beta}=q+\eta_{\alpha \beta}
$$

até termos de segunda ordem, de modo que a expansão de $f_{n}\left(m_{\alpha}, q_{\alpha \beta}\right)$ é dada por

$$
f_{n}\left(m_{\alpha}, q_{\alpha \beta}\right)=f_{n}(m, q)+\frac{1}{2} \frac{\partial^{2} f}{\partial m_{\alpha} \partial m_{\beta}} \epsilon_{\alpha} \epsilon_{\beta}+\frac{1}{2} \frac{\partial^{2} f}{\partial m_{\gamma} \partial q_{\alpha \beta}} \epsilon_{\gamma} \eta_{\alpha \beta}+\frac{1}{2} \frac{\partial^{2} f}{\partial q_{\alpha \beta} \partial q_{\delta \gamma}} \eta_{\alpha \beta} \eta_{\delta \gamma}+\cdots,
$$

em que as derivadas primeiras não aparecem, pois são nulas devido à condição de extremo. As derivadas de segunda ordem são tomadas em $m_{\alpha}=m$ e $q_{\alpha \beta}=q$ (solução réplica simétrica) e fornecem a matriz de estabilidade ou a hessiana. O cálculo dos elementos da matriz hessiana são feitos no apêndice $\mathrm{A}$.

A matriz hessiana tem a estrutura abaixo

$$
\mathbb{G}=\left(\begin{array}{cc}
G_{\alpha \beta} & G_{\gamma(\alpha \beta)} \\
G_{(\alpha \beta) \gamma} & G_{(\alpha \beta)(\delta \gamma)}
\end{array}\right)=\left(\begin{array}{cc}
\frac{\partial^{2} f_{n}}{\partial m_{\alpha} \partial m_{\beta}} & \frac{\partial^{2} f_{n}}{\partial m_{\gamma} \partial q_{\alpha \beta}} \\
\frac{\partial^{2} f_{n}}{\partial q_{\alpha \beta} \partial m_{\gamma}} & \frac{\partial^{2} f_{n}}{\partial q_{\alpha \beta} \partial q_{\delta \gamma}}
\end{array}\right),
$$

em que $G_{\alpha \beta}$ são matrizes $n \times n$ e $G_{\gamma(\alpha \beta)}$ são matrizes $n \times n(n-1) / 2$. Os elementos da matriz hessiana são classificados em sete tipos distintos. No caso $p=2$ e campo uniforme, recuperamos os elementos da matriz hessiana para o modelo SK (Almeida e Thouless, 1978) conforme podemos verificar no apêndice A.

Os autovalores da matriz hessiana $\mathbb{G}$ de ordem $n(n+1) / 2$ são dados pela equação característica

$$
\mathbb{G} \mu=\lambda \mu
$$

Com os autovetores escritos na forma

$$
\boldsymbol{\mu}=\left[\begin{array}{c}
\left\{\epsilon_{\alpha}\right\} \\
\left\{\eta_{(\alpha \beta)}\right\}
\end{array}\right]
$$

a equação de autovalores (2.65) pode ser escrita explicitamente como

$$
\begin{aligned}
& \sum_{\beta} G_{\alpha \beta} \epsilon_{\beta}+\sum_{(\beta \gamma)} G_{\alpha(\beta \gamma)} \eta_{(\beta \gamma)}=\lambda \epsilon_{\alpha} \\
& \sum_{\gamma} G_{(\alpha \beta) \gamma} \epsilon_{\gamma}+\sum_{(\delta \gamma)} G_{(\alpha \beta)(\delta \gamma)} \eta_{(\delta \gamma)}=\lambda \eta_{(\alpha \beta)}
\end{aligned}
$$


Utilizando as definições do apêndice A para os elementos da matriz hessiana as equações (2.67)-(2.68) ficam

$$
\begin{aligned}
& A \epsilon_{\alpha}+B \sum_{\beta \neq \alpha} \epsilon_{\beta}+C \sum_{\beta \neq \alpha} \eta_{(\alpha \beta)}+D \sum_{(\beta \gamma) \mid \beta, \gamma \neq \alpha} \eta_{(\beta \gamma)}=\lambda \epsilon_{\alpha}, \\
& C\left(\epsilon_{\alpha}+\epsilon_{\beta}\right)+D \sum_{\gamma \neq \alpha, \beta} \epsilon_{\gamma}+P \eta_{(\alpha \beta)}+Q \sum_{\gamma \neq(\alpha, \beta)}\left(\eta_{(\alpha \gamma)}+\eta_{(\beta \gamma)}\right)+R \sum_{(\gamma \delta) \mid \delta, \gamma \neq \alpha, \beta} \eta_{(\delta \gamma)}=\lambda \eta_{(\alpha \beta)} .
\end{aligned}
$$

Uma vez feito o cálculo dos autovalores, poderemos determinar os autovetores que dividem o espaço de dimensão $n(n+1) / 2$ em subespaços ortogonais (Almeida e Thouless, 1978). Os $n(n+1) / 2$ autovetores linearmente independentes podem ser classificados em longitudinais, anômalos e transversais (Dominicis e Kondor, 1983).

\subsubsection{Os autovetores longitudinais}

Os autovetores longitudinais $\left(\boldsymbol{\mu}^{L}\right)$ são aqueles cujos elementos independem dos índices das réplicas, isto é

$$
\begin{aligned}
& \epsilon_{\alpha}^{L}=a, \quad \text { para todo } \alpha \\
& \eta_{(\alpha \beta)}^{L}=b, \quad \text { para todo os pares distintos }(\alpha \beta) \text {. }
\end{aligned}
$$

Substituindo nas equações (2.69)-(2.70), obtemos o seguinte sistema de equações para os autovalores longitudinais

$$
\left[\begin{array}{cc}
A+(n-1) B & (n-1) C+\frac{1}{2}(n-1)(n-2) D \\
2 C+(n-2) D & P+2(n-2) Q+\frac{1}{2}(n-2)(n-3) R
\end{array}\right] \times\left[\begin{array}{l}
a \\
b
\end{array}\right]=\lambda\left[\begin{array}{l}
a \\
b
\end{array}\right]
$$

Os autovetores longitudinais geram o subespaço com dimensão 2.

\subsubsection{Os autovetores anômalos}

Os autovetores anômalos são aqueles que dependem apenas de um único índice de réplica e são dados por

$$
\begin{aligned}
\epsilon_{\alpha}^{A} & =d_{\alpha}, \quad \text { para todo } \alpha \\
\eta_{(\alpha \beta)}^{A} & =\frac{1}{2}\left(e_{\alpha}+e_{\beta}\right), \quad \text { para todo os pares distintos }(\alpha \beta)
\end{aligned}
$$


Impondo a condição de que os autovetores anômalos $\mu^{A}$ sejam ortogonais aos autovetores longitudinais $\mu^{L}$, temos

$$
\begin{aligned}
\mu^{A} \cdot \mu^{L} & =\sum_{\alpha} \epsilon_{\alpha}^{L} \epsilon_{\alpha}^{A}+\sum_{(\alpha \beta)} \eta_{(\alpha \beta)}^{L} \eta_{(\alpha \beta)}^{A} \\
& =a\left(\sum_{\alpha} d_{\alpha}\right)+\frac{(n-1) b}{2}\left(\sum_{\alpha} e_{\alpha}\right)=0
\end{aligned}
$$

Portanto, temos da condição acima que

$$
\sum_{\alpha} d_{\alpha}=\sum_{\alpha} e_{\alpha}=0
$$

Utilizando a definição de autovetores anômalos e a relação $(2.77)$, temos

$$
\begin{aligned}
\sum_{\beta \neq \alpha} d_{\beta} & =\sum_{\beta} d_{\beta}-d_{\alpha}=-d_{\alpha} \\
\sum_{(\beta \alpha) \mid \beta, \gamma \neq \alpha}\left(e_{\beta}+e_{\gamma}\right) & =(n-2) \sum_{\beta \neq \gamma} e_{\beta}=(n-2)\left(\sum_{\beta} e_{\beta}-e_{\alpha}\right)=-(n-2) e_{\alpha} .
\end{aligned}
$$

Deste modo, a equação de autovalores pode ser escrita como

$$
\left[\begin{array}{cc}
A-B & \frac{(n-2)}{2}(C-D) \\
2(C-D) & P+(n-4) Q-(n-3) R
\end{array}\right]\left[\begin{array}{l}
d_{\alpha} \\
e_{\alpha}
\end{array}\right]=\lambda\left[\begin{array}{l}
d_{\alpha} \\
e_{\alpha}
\end{array}\right]
$$

O número de autovetores anômalos linearmente independentes correspondentes ao dois autovalores da equação $(2.80)$ é $2(n-1)$, sendo que cada um deles é $(n-1)$ vezes degenerado. Portanto, o número de autovetores linearmente independentes gerado pelos autovalores longitudinais e anômalos é igual $2 n$.

\subsubsection{Os autovetores transversais}

Os autovetores transversais são aqueles cujos elementos dependem dos dois índices de réplicas,

$$
\begin{aligned}
\epsilon_{\alpha}^{T} & =0, \quad \text { para todo } \alpha \\
\eta_{(\alpha \beta)}^{T} & =h_{(\alpha \beta)}, \quad \text { para todo } \quad(\alpha \beta) .
\end{aligned}
$$


Queremos que esses autovetores sejam linearmente independentes dos autovetores longitudinais $\mu^{L}$ e transversais $\mu^{A}$. Portanto, impondo

$$
\begin{aligned}
\mu^{L} \cdot \mu^{T} & =\frac{b}{2} \sum_{\alpha}\left(\sum_{\beta \neq \alpha} h_{(\alpha \beta)}\right)=0, \\
\mu^{A} \cdot \mu^{T} & =\frac{1}{2} \sum_{\alpha} e_{\alpha}\left(\sum_{\beta \neq \alpha} h_{(\alpha \beta)}\right)=0 .
\end{aligned}
$$

A condição de ortogonalidade implica

$$
\sum_{\beta \neq \alpha} h_{(\alpha \beta)}=0
$$

Deste modo, temos

$$
\sum_{(\beta \gamma) \mid \beta, \gamma \neq \alpha} h_{(\alpha \beta)}=\frac{1}{2} \sum_{\beta \neq \alpha}\left(\sum_{\gamma \neq \beta} h_{(\beta \gamma)}-h_{(\alpha \beta)}\right)=-\frac{1}{2} \sum_{\beta \neq \alpha} h_{(\alpha \beta)}=0
$$

Substituindo em (2.69) e (2.70), obtemos

$$
P h_{(\alpha \beta)}+Q \sum_{\gamma \neq \alpha, \beta}\left(h_{(\alpha \gamma)}+h_{(\beta \gamma)}\right) R+\sum_{(\delta \gamma) \mid \delta \gamma \neq \alpha, \beta} h_{(\gamma \delta)}=\lambda h_{(\alpha \beta)}
$$

em que

$$
\sum_{\gamma \neq \alpha, \beta} h_{(\alpha \gamma)}=\sum_{\gamma \neq \alpha} h_{(\alpha \gamma)}-h_{(\alpha \beta)}=-h_{(\alpha \beta)}
$$

Portanto,

$$
\lambda=P-2 Q+R
$$

A este autovalor correspondem $n(n-3) / 2$ autovetores transversais linearmente independentes. Observamos que a soma dos números de autovetores linearmente independentes de cada subespaço é igual à ordem da matriz hessiana.

\subsubsection{Condição de estabilidade}

A condição necessária e suficiente para a estabilidade da solução réplica simétrica é que os autovalores da matriz hessiana sejam positivos para qualquer valor de $n$ (inclusive para $n \rightarrow 0$ que é a continuação analítica da solução).

Para os autovalores transversais, para qualquer valor de $n$, a condição de estabilidade é dada por

$$
\lambda=P-2 Q+R>0
$$


válida inclusive para $n=0$, pois a expressão independe de $n$.

Contudo, no limite $p \rightarrow \infty, P, Q$ e $R$ são iguais a zero. Os dois únicos elementos da matriz hessiana que não são zero quando tomado o limite $p \rightarrow \infty$ são $A$ e $B$. Portanto, em cada subespaço (longitudinal, anômalo e transverso) surgiram autovalores que serão zero e a fase é marginalmente estável. Faz-se necessário considerar as soluções com um passo de quebra de simetria de réplica proposto por Parisi de modo a obter o diagrama de fases completo para o modelo.

\subsection{Quebra de Simetria entre Réplicas}

Após tentativas de vários pesquisadores, Parisi propôs um esquema de quebra de simetria entre réplicas para a matriz $q_{\alpha \beta}$ que produz uma solução de campo médio estável para o modelo SK (Dominicis e Kondor, 1983). Na solução de Parisi, temos que fazer infinitas quebras de simetria (Parisi, 1979, 1980b, 1980a) a fim de obter uma solução correta para o modelo SK. No caso do modelo de energias aleatórias Gross e Mézard mostraram que um único passo de quebra de simetria já era suficiente para garantir uma solução correta do modelo, quando este era tratado pelo método de réplicas (Gross e Mézard, 1984). A parametrização de Parisi, para um único passo de quebra de simetria, consiste em dividir a matriz $n \times n$ em $n / m_{1}$ grupos de $m_{1}$ réplicas. Naturalmente $n$ deve ser múltiplo de $m_{1}$. Os $q_{\alpha \beta}=q_{1}$ se $\alpha$ e $\beta$ pertencem ao mesmo grupo e $q_{\alpha \beta}=q_{0}$, se $\alpha$ e $\beta$ pertencem a grupos diferentes. Os $q_{\alpha \alpha}$ (elementos da diagonal) são identicamente nulos. O Ansatz de Parisi pode ser escrito como

$$
\begin{aligned}
& m_{\alpha}=m, \\
& q_{\alpha \beta \beta}= \begin{cases}0 & \text { se } \alpha=\beta \\
q_{1} & \text { se }\left[\alpha / m_{1}\right]=\left[\beta / m_{1}\right] \text { e } \alpha \neq \beta \\
q_{0} & \text { caso contrário, }\end{cases}
\end{aligned}
$$

em que $[x]$ é o menor inteiro maior ou igual a $x$.

Por exemplo, a matriz $\left(q_{\alpha \beta}\right)$ no caso de um passo de quebra com $n=6$ e $m_{1}=3$ tem 
a forma

$$
q_{\alpha \beta}=\left[\begin{array}{ccc|ccc}
0 & q_{1} & q_{1} & q_{0} & q_{0} & q_{0} \\
q_{1} & 0 & q_{1} & q_{0} & q_{0} & q_{0} \\
q_{1} & q_{1} & 0 & q_{0} & q_{0} & q_{0} \\
\hline q_{0} & q_{0} & q_{0} & 0 & q_{1} & q_{1} \\
q_{0} & q_{0} & q_{0} & q_{1} & 0 & q_{1} \\
q_{0} & q_{0} & q_{0} & q_{1} & q_{1} & 0
\end{array}\right]
$$

Como vimos em (2.34) a energia livre por spin é dada por

$$
\beta f=-\frac{(\beta J)^{2}}{4}+\lim _{n \rightarrow 0} \frac{1}{n}\left[\frac{(\beta J)^{2}}{2}(p-1) \sum_{\alpha<\beta} q_{\alpha \beta}^{p}+\frac{\beta J_{0}}{2} \sum_{\alpha} m_{\alpha}^{2}-\left\langle\ln \operatorname{Tr} e^{\overline{\mathcal{H}}}\right\rangle_{H}\right] .
$$

em que a hamiltoniana efetiva é igual a

$$
\overline{\mathcal{H}}=\frac{(\beta J)^{2}}{2} p \sum_{\alpha<\beta} q_{\alpha \beta}^{p-1} S^{\alpha} S^{\beta}+\sum_{\alpha} \beta\left(H+J_{0} m_{\alpha}\right) S^{\alpha} .
$$

Para um passo de quebra de simetria entre réplicas, temos

$$
\sum_{\alpha<\beta} q_{\alpha \beta}^{p}=\frac{1}{2} \sum_{\alpha \neq \beta} q_{\alpha \beta}^{p}=\frac{n}{2}\left[\left(n-m_{1}\right) q_{0}^{p}+\left(m_{1}-1\right) q_{1}^{p}\right]
$$

O primeiro termo da hamiltoniana efetiva (2.95), fica

$$
\begin{aligned}
\sum_{\alpha<\beta} q_{\alpha \beta}^{p-1} S^{\alpha} S^{\beta}= & \frac{1}{2} \sum_{\alpha \neq \beta} q_{\alpha \beta}^{p-1} S^{\alpha} S^{\beta}=\frac{1}{2} q_{0}^{p-1}\left(\sum_{\alpha} S^{\alpha}\right)^{2}+\frac{q_{1}^{p-1}-q_{0}^{p-1}}{2} \sum_{k=1}^{n / m_{1}}\left(\sum_{\alpha=(k-1) m_{1}+1}^{k m_{1}} S^{\alpha}\right)^{2} \\
& -\frac{q_{1}^{p-1}}{2} \sum_{\alpha}\left(S^{\alpha}\right)^{2} .
\end{aligned}
$$

Essa identidade pode ser mais facilmente visualizada se observarmos que a matriz $\left(q_{\alpha \beta}\right)$ 
pode ser decomposta como no exemplo abaixo,

$$
\begin{aligned}
& q_{\alpha \beta}= {\left[\begin{array}{cc|cc|cc}
0 & q_{1} & q_{0} & q_{0} & q_{0} & q_{0} \\
q_{1} & 0 & q_{0} & q_{0} & q_{0} & q_{0} \\
\hline q_{0} & q_{0} & 0 & q_{1} & q_{0} & q_{0} \\
q_{0} & q_{0} & q_{1} & 0 & q_{0} & q_{0} \\
\hline q_{0} & q_{0} & q_{0} & q_{0} & 0 & q_{1} \\
q_{0} & q_{0} & q_{0} & q_{0} & q_{1} & 0
\end{array}\right]=q_{0}\left[\begin{array}{cc|cc|cc}
1 & 1 & 1 & 1 & 1 & 1 \\
1 & 1 & 1 & 1 & 1 & 1 \\
\hline 1 & 1 & 1 & 1 & 1 & 1 \\
1 & 1 & 1 & 1 & 1 & 1 \\
\hline 1 & 1 & 1 & 1 & 1 & 1 \\
1 & 1 & 1 & 1 & 1 & 1
\end{array}\right] } \\
&\left.+\left(q_{1}-q_{0}\right)\left[\begin{array}{ll|ll|ll}
1 & 1 & 0 & 0 & 0 & 0 \\
1 & 1 & 0 & 0 & 0 & 0 \\
\hline 0 & 0 & 1 & 1 & 0 & 0 \\
0 & 0 & 1 & 1 & 0 & 0 \\
\hline 0 & 0 & 0 & 0 & 1 & 1 \\
0 & 0 & 0 & 0 & 1 & 1
\end{array}\right]+q_{1} \mid \begin{array}{ll|ll|ll}
1 & 0 & 0 & 0 & 0 & 0 \\
0 & 1 & 0 & 0 & 0 & 0 \\
\hline 0 & 0 & 1 & 0 & 0 & 0 \\
0 & 0 & 1 & 0 & 0 \\
\hline 0 & 0 & 0 & 0 & 1 & 0 \\
0 & 0 & 0 & 0 & 0 & 1
\end{array}\right] .
\end{aligned}
$$

Portanto, a hamiltoniana efetiva (2.95) pode ser escrita como

$$
\begin{aligned}
\overline{\mathcal{H}}= & \sum_{\alpha} \beta\left(H+J_{0} m_{\alpha}\right) S^{\alpha}+\frac{(\beta J)^{2}}{4} p\left[q_{0}^{p-1}\left(\sum_{\alpha} S^{\alpha}\right)^{2}+\left(q_{1}^{p-1}-q_{0}^{p-1}\right) \sum_{k=1}^{n / m_{1}}\left(\sum_{\alpha=(k-1) m_{1}+1}^{k m_{1}} S^{\alpha}\right)^{2}\right. \\
& \left.-q_{1}^{p-1} n\right] .
\end{aligned}
$$

Os termos quadráticos na hamiltoniana efetiva (2.99) podem ser linearizados por meio da identidade gaussiana

$$
e^{a^{2} / 2}=\int_{-\infty}^{\infty} \frac{d x}{\sqrt{2 \pi}} e^{-x^{2} / 2 \pm a x}
$$

de modo que

$$
\operatorname{Tr} \exp \overline{\mathcal{H}}=\exp \left[\frac{(\beta J)^{2} p q_{1}^{p-1} n}{4}\right] \int_{-\infty}^{\infty} D x\left[Z_{0}(x)\right]^{n / m_{1}},
$$

em que $D x=(d x / \sqrt{2 \pi}) e^{-x^{2} / 2} \cdot Z_{0}(x)$ pode ser vista como a função de partição do sistema no espaço de réplicas, e é dada por

$$
Z_{0}(x)=\operatorname{Tr} \exp \left[\beta\left(H+J_{0} m+J \sqrt{\frac{p}{2} q_{0}^{p-1}} x\right)\left(\sum_{\alpha=1}^{m_{1}} S^{\alpha}\right)+\frac{(\beta J)^{2}}{4} p\left(q_{1}^{p-1}-q_{0}^{p-1}\right)\left(\sum_{\alpha=1}^{m_{1}} S^{\alpha}\right)^{2}\right],
$$


Aplicando novamente a identidade gaussiana em (2.100), obtemos

$$
Z_{0}(x)=\int_{-\infty}^{\infty} D y\left[Z_{1}(y)\right]^{m_{1}}
$$

Para spins do tipo Ising, temos

$$
Z_{1}(y)=2 \cosh \bar{H}
$$

em que

$$
\bar{H}=\beta\left(H+J_{0} m+J \sqrt{\frac{p}{2} q_{0}^{p-1}} x+J \sqrt{\frac{p}{2}\left(q_{1}^{p-1}-q_{0}^{p-1}\right) y}\right) .
$$

Portanto, a energia livre para um passo de quebra de simetria entre réplicas é dada por $\beta f=\frac{\beta J_{0}}{2} m^{2}-\frac{(\beta J)^{2}}{4}\left\{1+(p-1)\left[m_{1} q_{0}^{p}+\left(1-m_{1}\right) q_{q}^{p}\right]-p q_{1}^{p-1}\right\}-\frac{1}{m_{1}} \int_{-\infty}^{\infty} D x\left\langle\ln Z_{0}(x)\right\rangle_{H}$.

As equações de extremos são dadas por

$$
\begin{aligned}
\frac{\partial(\beta f)}{\partial m_{1}}= & \frac{(\beta J)^{2}}{4}\left[q_{1}^{p}-(p-1) q_{0}^{p}\right]+\frac{1}{m_{1}^{2}} \int_{-\infty}^{\infty} D x\left\langle\ln Z_{0}(x)\right\rangle_{H} \\
& -\frac{1}{m_{1}} \int_{-\infty}^{\infty} D x\left\langle\frac{1}{Z_{0}(x)} \int_{-\infty}^{\infty} D y\left[Z_{1}(y)\right]^{m_{1}} \ln Z_{1}(y)\right\rangle_{H}=0 \\
\frac{\partial(\beta f)}{\partial m}= & \beta J_{0} m-\beta J_{0} m \int_{-\infty}^{\infty} D x\left\langle\frac{1}{Z_{0}(x)} \int_{-\infty}^{\infty} D y\left[Z_{1}(y)\right]^{m_{1}} \tanh \bar{H}\right\rangle_{H}=0 \\
\frac{\partial(\beta f)}{\partial q_{0}}= & -\frac{(\beta J)^{2}}{4} p(p-1) m_{1} q_{0}^{p-1}-\frac{(\beta J)^{2}}{4} p(p-1) m_{1} q_{0}^{p-2}\left[1-\left(1-m_{1}\right) q_{1}\right. \\
& \left.-m_{1} \int_{-\infty}^{\infty} D x\left\langle\left(\frac{1}{Z_{0}(x)} \int_{-\infty}^{\infty} D y\left[Z_{1}(y)\right]^{m_{1}} \tanh \bar{H}\right)^{2}\right\rangle_{H}\right]+\frac{(\beta J)^{2}}{4} p(p-1) \\
& \times\left[1-\left(1-m_{1}\right) q_{1}\right] q_{0}^{p-2}=0, \\
\frac{\partial(\beta f)}{\partial q_{1}}= & \frac{(\beta J)^{2}}{4}\left[p(p-1)\left(1-m_{1}\right) q_{1}^{p-1}-p(p-1) q_{1}^{p-2}\right]-\frac{(\beta J)^{2}}{4} p(p-1) q_{1}^{p-2} \\
& \times \int_{-\infty}^{\infty} D x\left\langle\frac{1}{Z(x)} \int_{-\infty}^{\infty} D y\left[Z_{1}(y)\right]^{m_{1}} \tanh ^{2} \bar{H}\right\rangle_{H}=0 .
\end{aligned}
$$

Vamos inicialmente o caso $0 \leq q_{0} \leq q_{1}<1$, no limite $p \rightarrow \infty$. Neste limite

$$
\begin{aligned}
& Z_{1}=2 \cosh \beta\left(H+J_{0} m\right), \\
& Z_{0}=\left[2 \cosh \beta\left(H+J_{0} m\right)\right]^{m_{1}} .
\end{aligned}
$$

Os parâmetros $q_{0}$ e $m$ são dados por

$$
\begin{aligned}
& q_{0}=\left\langle\tanh ^{2} \beta\left(H+J_{0} m\right)\right\rangle_{H}, \\
& m=\left\langle\tanh \beta\left(H+J_{0} m\right)\right\rangle_{H} .
\end{aligned}
$$


Vemos que esses resultados coincidem com a solução réplica simétrica. Consideramos o caso $0 \leq q_{0}<q_{1}=1$. No limite $p \rightarrow \infty$,

$$
\begin{aligned}
& Z_{1}=2 \cosh \beta \bar{H}_{y}, \quad \text { com } \quad \bar{H}_{y}=\beta\left(H+J_{0} m+J \sqrt{\frac{p}{2}} y\right) \\
& Z_{0}=\int_{-\infty}^{\infty} D y\left[Z_{1}(y)\right]^{m_{1}}=\int_{-\infty}^{\infty} D y\left[2 \cosh \beta\left(H+J_{0} m+J \sqrt{\frac{p}{2}} y\right)\right]^{m_{1}}
\end{aligned}
$$

O comportamento assintótico de $Z_{0}$ é dado por

$$
Z_{0}(x) \sim 2 \cosh \left[\beta\left(H+J_{0} m\right) m_{1}\right] \exp \left[\frac{(\beta J)^{2}}{4} p m_{1}\right]
$$

E a energia livre por spin é dada por

$$
f=\frac{J_{0} m^{2}}{2}-\frac{\beta J^{2} m_{1}}{4}-\frac{1}{\beta m_{1}}\left\langle\ln 2 \cosh \left[\beta\left(H+J_{0} m\right) m_{1}\right]\right\rangle_{H}
$$

Com os parâmetros $m$ e $q$ dados por

$$
\begin{aligned}
m & =\left\langle\tanh \beta\left(H+J_{0} m\right) m_{1}\right\rangle_{H} \\
q_{0} & =\left\langle\tanh ^{2} \beta\left(H+J_{0} m\right) m_{1}\right\rangle_{H}
\end{aligned}
$$

A equação que determina $m_{1}$ é dada por

$$
\frac{(\beta J)^{2} m_{1}^{2}}{4}=\left\langle\ln 2 \cosh \beta\left(H+J_{0} m\right) m_{1}\right\rangle_{H}-\left\langle\beta\left(H+J_{0} m\right) \tanh \beta\left(H+J_{0} m\right) m_{1}\right\rangle_{H}
$$

Essa equação determina o produto $\beta m_{1}=\beta_{c}$. Para $\beta<\beta_{c} m=1$ e a solução réplica simétrica é correta, enquanto para $\beta>\beta_{c}$ o sistema fica congelado com entropia igual a zero.

\subsection{Caso $J_{0}=0$ : ausência de interação ferromagnética entre pares}

No caso $J_{0}=0$, temos o modelo de energias aleatórias, sem interação ferromagnética, e na presença de um campo aleatório. A energia livre por spins é dada por

$$
\beta f=-\frac{(\beta J)^{2}}{4}-\langle\ln 2 \cosh \beta H\rangle_{H} .
$$

A energia interna por spin é

$$
u=-\frac{(\beta J)^{2}}{2}-\langle H \tanh \beta H\rangle_{H},
$$


e a entropia por spins é dada por

$$
\frac{s}{k_{B}}=-\frac{(\beta J)^{2}}{4}+\langle\ln 2 \cosh \beta H\rangle_{H}-\beta\langle H \tanh \beta H\rangle_{H}
$$

Para um campo magnético uniforme a distribuição de campo externo é dada por

$$
\wp(H)=\delta\left(H-H_{0}\right)
$$

Adotando unidades tais que $k_{B}=1$ e $J=1$ temos

$$
f=-\frac{1}{4 T}-T \ln 2 \cosh \left(\frac{H_{0}}{T}\right)
$$

e

$$
s=-\frac{1}{4 T^{2}}+\ln 2 \cosh \left(\frac{H_{0}}{T}\right)-\frac{H_{0}}{T} \tanh \left(\frac{H_{0}}{T}\right) .
$$

Portanto, a temperatura em que a entropia é zero é dada por

$$
T=\frac{1}{2 \sqrt{\ln 2 \cosh x-x \tanh x}}
$$

com $H_{0}=T x$. Fazendo $H_{0}=0$, campo externo nulo, $T_{c}=1 / 2 \sqrt{\ln 2}$, reobtendo o resultado de Derrida (Derrida, 1981).

\subsubsection{Distribuição duplo-delta}

A distribuição de campo é

$$
\wp(H)=\frac{1}{2} \delta\left(H-H_{0}-\sigma\right)+\frac{1}{2} \delta\left(H-H_{0}+\sigma\right)
$$

com as funções termodinâmicas dadas por

$$
f=-\frac{1}{4 T}-\frac{T}{2}\left[\ln 2 \cosh \left(\frac{H_{0}+\sigma}{T}\right)+\ln 2 \cosh \left(\frac{H_{0}-\sigma}{T}\right)\right],
$$

e

$$
\begin{aligned}
s= & -\frac{1}{4 T^{2}}+\frac{1}{2}\left[\ln 2 \cosh \left(\frac{H_{0}+\sigma}{T}\right)+\ln 2 \cosh \left(\frac{H_{0}-\sigma}{T}\right)\right] \\
& -\frac{1}{2 T}\left[\left(H_{0}+\sigma\right) \tanh \left(\frac{H_{0}+\sigma}{T}\right)+\left(H_{0}-\sigma\right) \tanh \left(\frac{H_{0}-\sigma}{T}\right)\right] .
\end{aligned}
$$


O diagrama de fase campo $\times$ temperatura para uma distribuição duplo-delta: $\wp(H)=\frac{1}{2} \delta\left(H-H_{0}-\sigma\right)+\frac{1}{2} \delta\left(H-H_{0}+\sigma\right)$ e interação ferromagnética $J_{0}=0$ é mostrado na figura 2.1 .

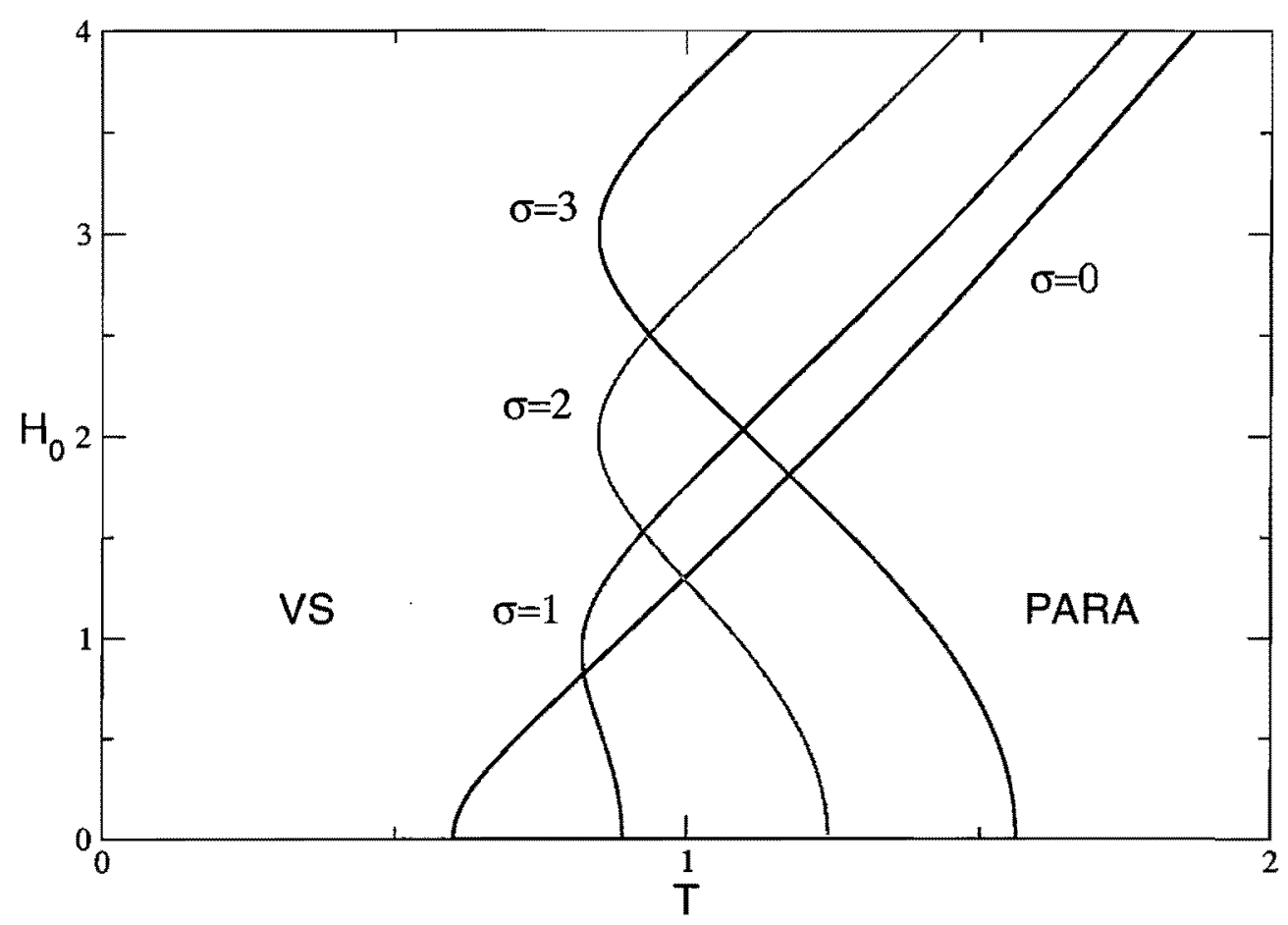

Figura 2.1: Diagrama de campo $\times$ temperatura para uma distribuição duplo-delta com $J_{0}=0$.

O diagrama 2.1 é obtido para valores $H_{0} \times T$ em que a entropia é igual a zero. $O$ valor $\sigma=0$ corresponde ao caso de campo uniforme obtido por (Derrida, 1981). Observamos um comportamento reentrante à medida que aumentamos o valor da variância $\sigma$. Na região vidro de spin (VS) o sistema está congelado no seu estado em $T=T_{c}$ com com entropia zero. 


\subsubsection{Distribuição de campo gaussiano (normal)}

Para um campo aleatório normal com média $H_{0}$ e variância $\sigma^{2}, H \sim N\left(H_{0}, \sigma^{2}\right)$, a distribuição de probabilidade é

$$
\wp(H)=\frac{1}{\sqrt{2 \pi} \sigma} \exp \left[-\frac{\left(H-H_{0}\right)^{2}}{2 \sigma^{2}}\right] .
$$

Os diagramas de fases campo $\times$ temperatura são mostrado na figura 2.2

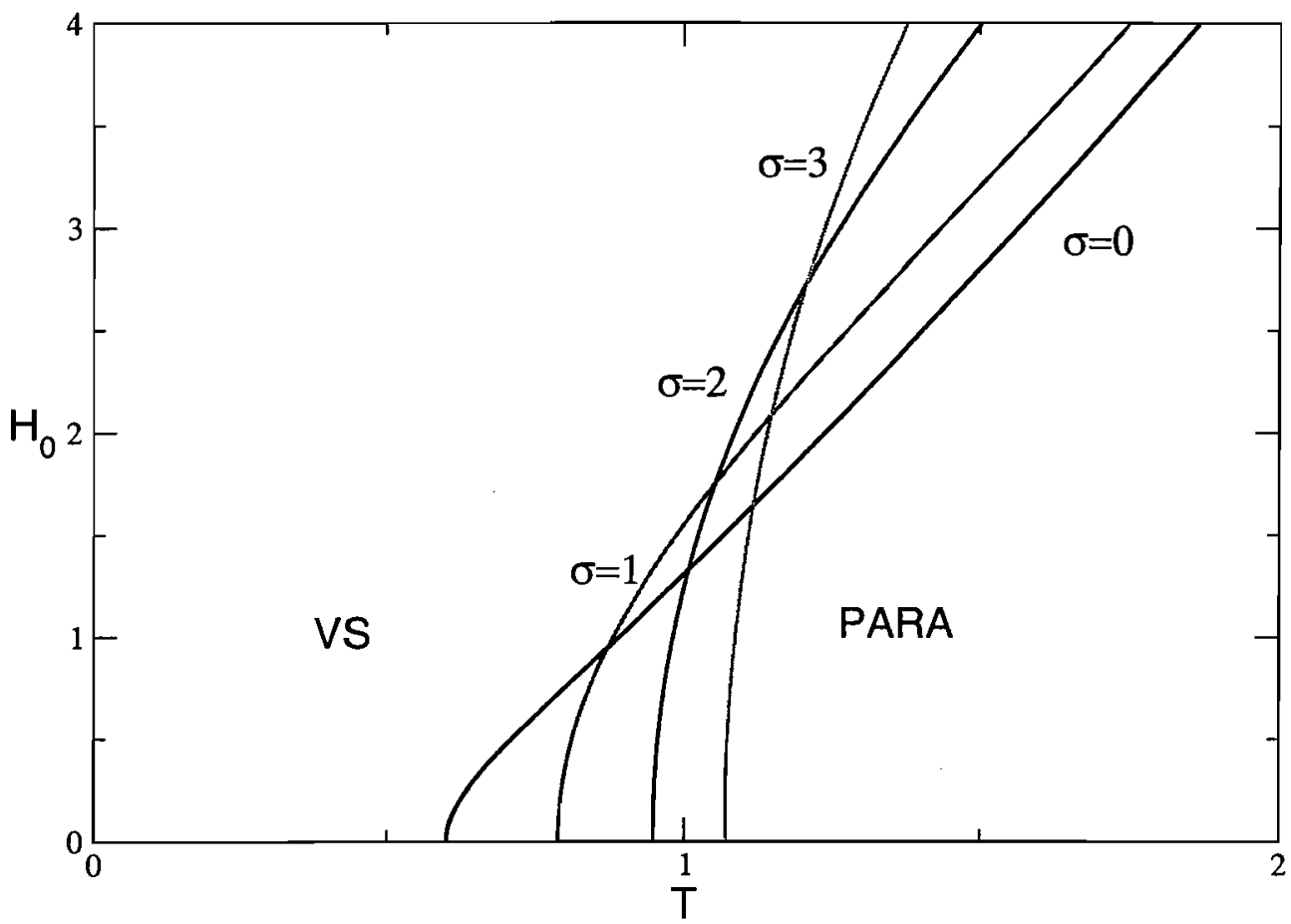

Figura 2.2: Diagrama campo $\times$ temperatura para uma distribuição normal com $J_{0}=0$ 


\subsection{Caso $J_{0}>0$ : com interação ferromagnética entre pares}

Neste caso, temos

$$
\bar{H}=\beta\left(H+J_{0} m\right)
$$

A magnetização será dada por

$$
m=\langle\tanh \bar{H}\rangle_{H}
$$

Diagrama de fase para campo zero é mostrado na figura 2.3.

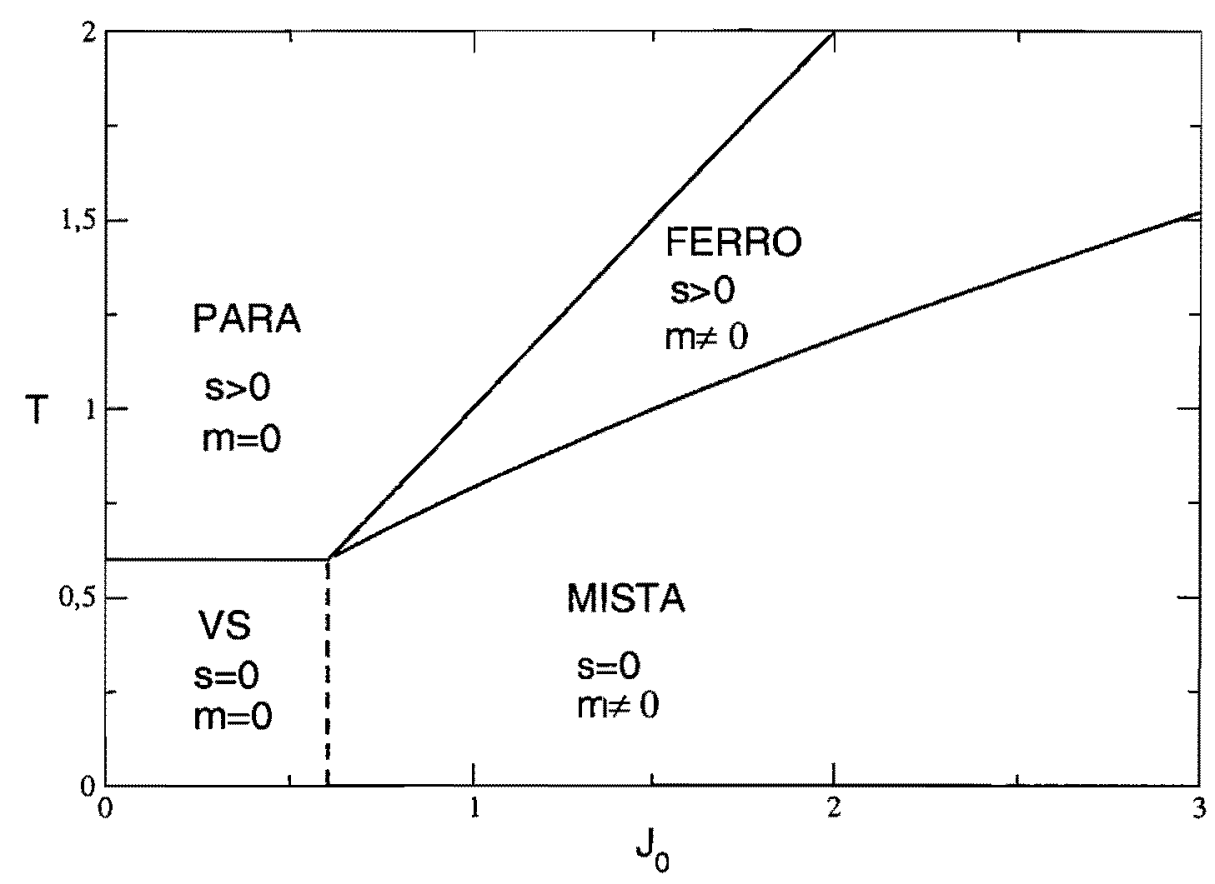

Figura 2.3: diagrama de fases para campo zero

No diagrama (2.3) a linha que separa a fase paramagnética da fase vidro de spin (VS) é obtida para magnetização zero e entropia nula. A linha entre a fase ferro e paramagnética é obtida para magnetização zero. A linha que separa a fase ferromagnética e mista é obtida para magnetização diferente de zero e entropia igual a zero. A fase mista tem este nome, pois combina característica da fase ferro $(m \neq 0)$ e entropia zero. 
Diagrama de fases para distribuição de campo aleatória duplo-delta com $\sigma=0,2$ é mostrado na figura 2.4 .

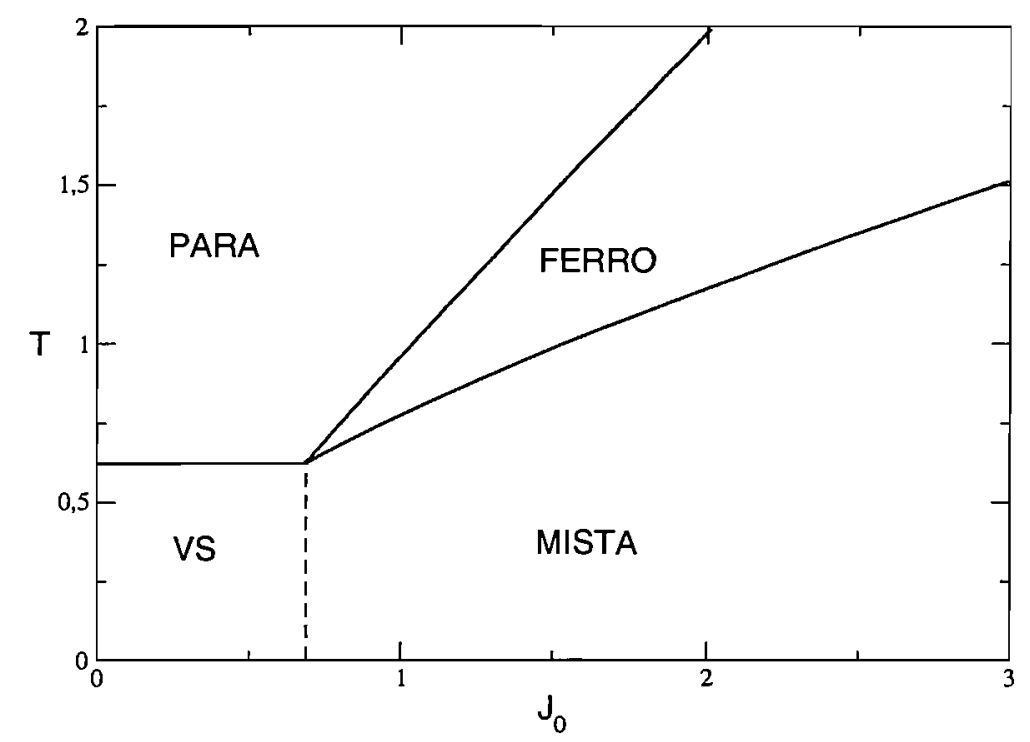

Figura 2.4: diagrama de fases para uma distribuição duplo-delta com $\sigma=0,2$

Diagrama de fases para distribuição de campo aleatória gaussiana com $\sigma=0,2$ é mostrado na figura 2.5.

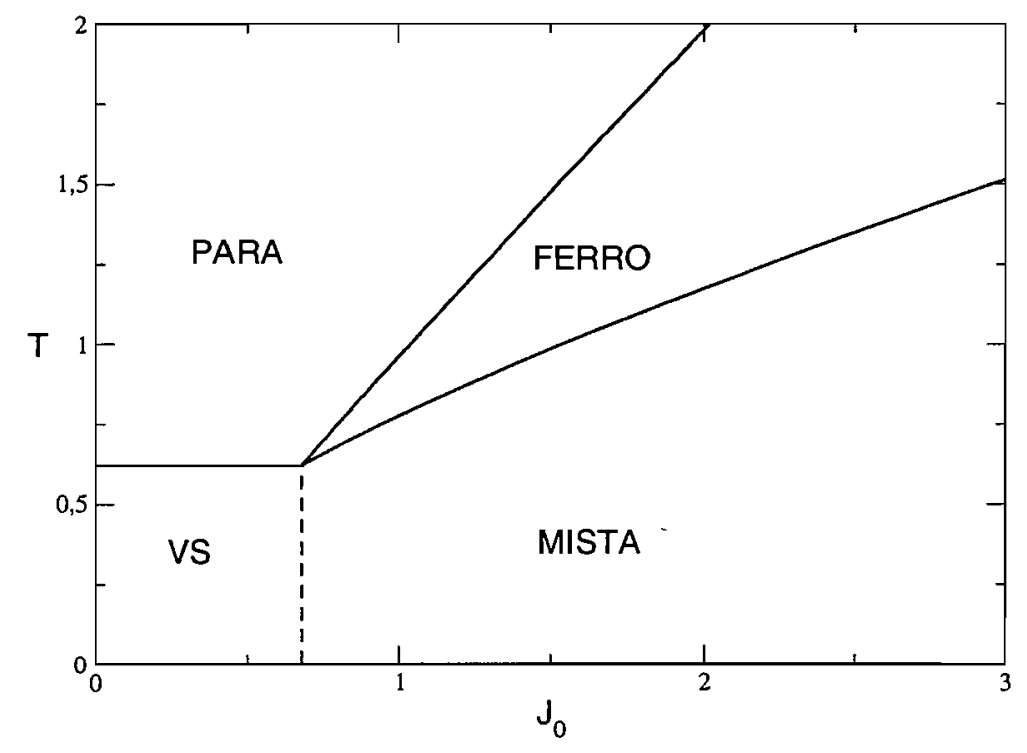

Figura 2.5: diagrama de fases para uma distribuição gaussiana com $\sigma=0,2$ 
Diagrama de fases para distribuição de campo aleatória duplo-delta com $\sigma=0,4$ é mostrado na figura 2.6.

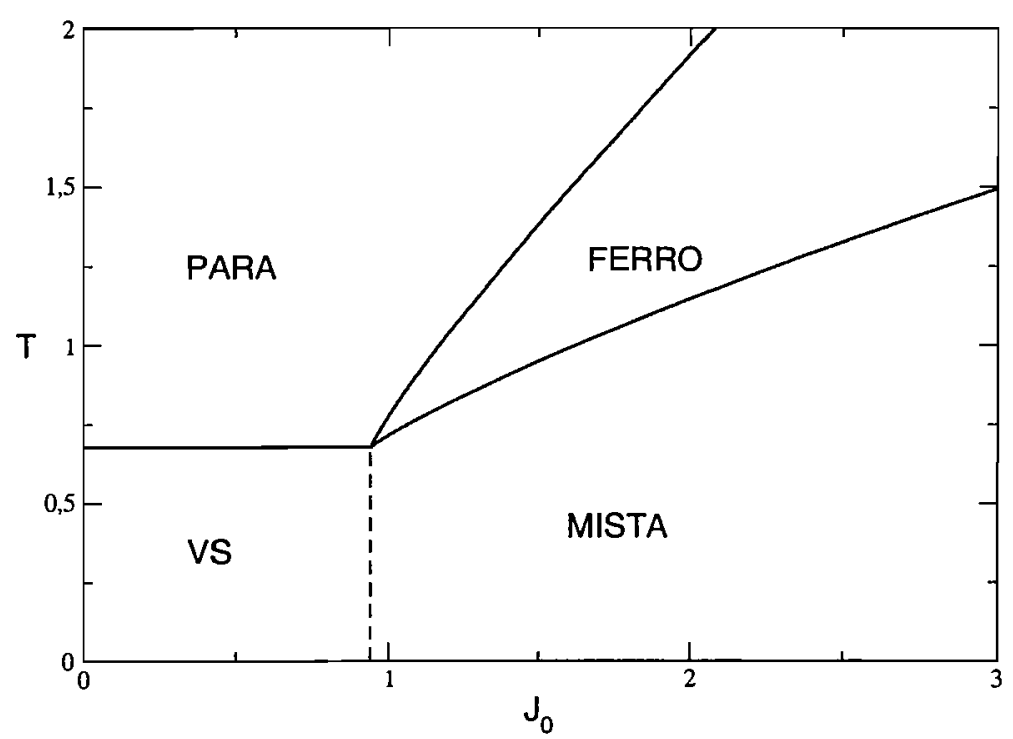

Figura 2.6: diagrama de fases para uma distribuição duplo-delta com $\sigma=0,4$

Diagrama de fases para distribuicão de campo aleatória normal com $\sigma=0,4$ é mostrado na figura 2.7

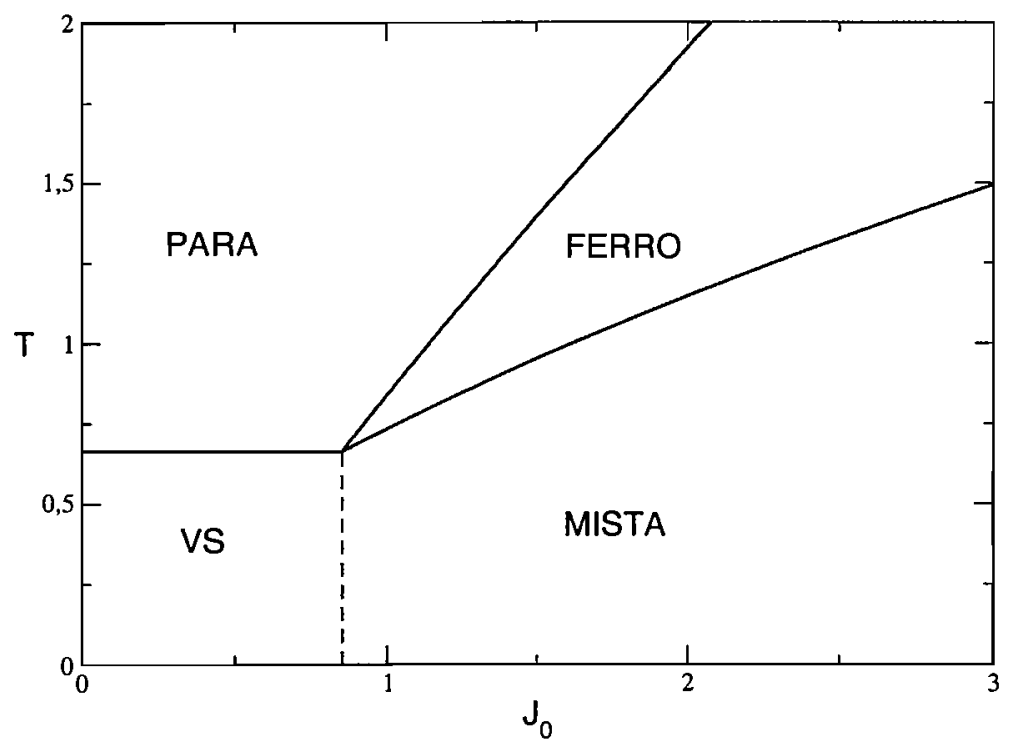

Figura 2.7: diagrama de fases para uma distribuição normal com $\sigma=0,4$ 
Diagrama de fases para distribuição de campo aleatória duplo-delta com $\sigma=0,8$ é mostrado na figura 2.8 .

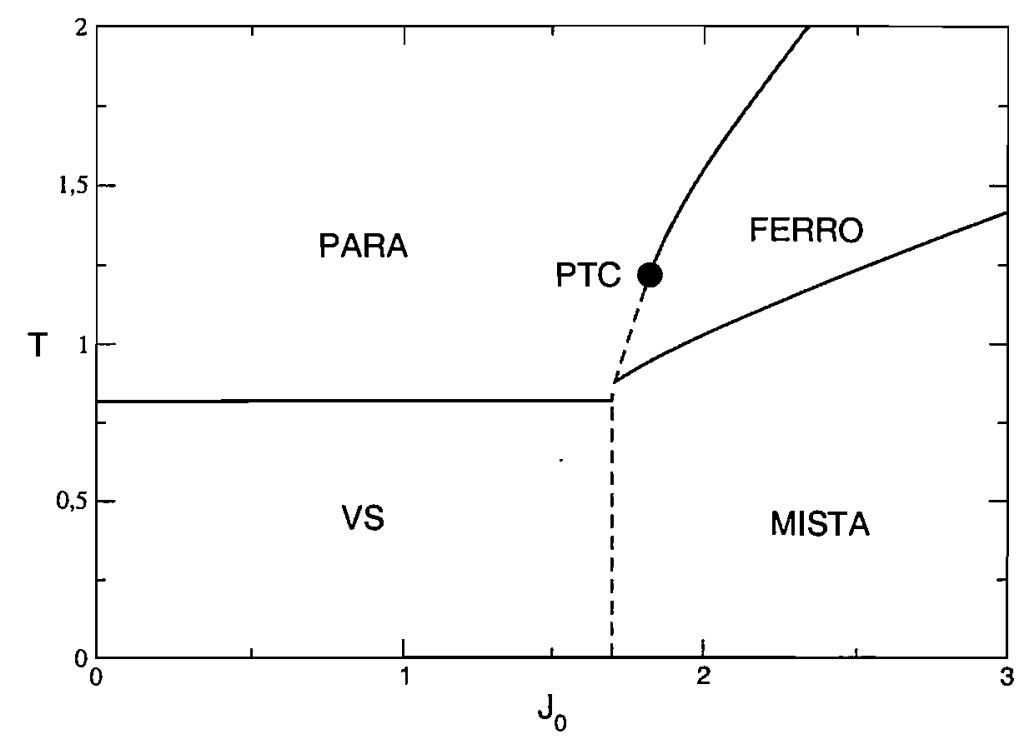

Figura 2.8: diagrama de fases para uma distribuição duplo-delta com $\sigma=0,8$

O diagrama de fases para uma distribuição de normal com $\sigma=0,8$ é mostrado na figura 2.9

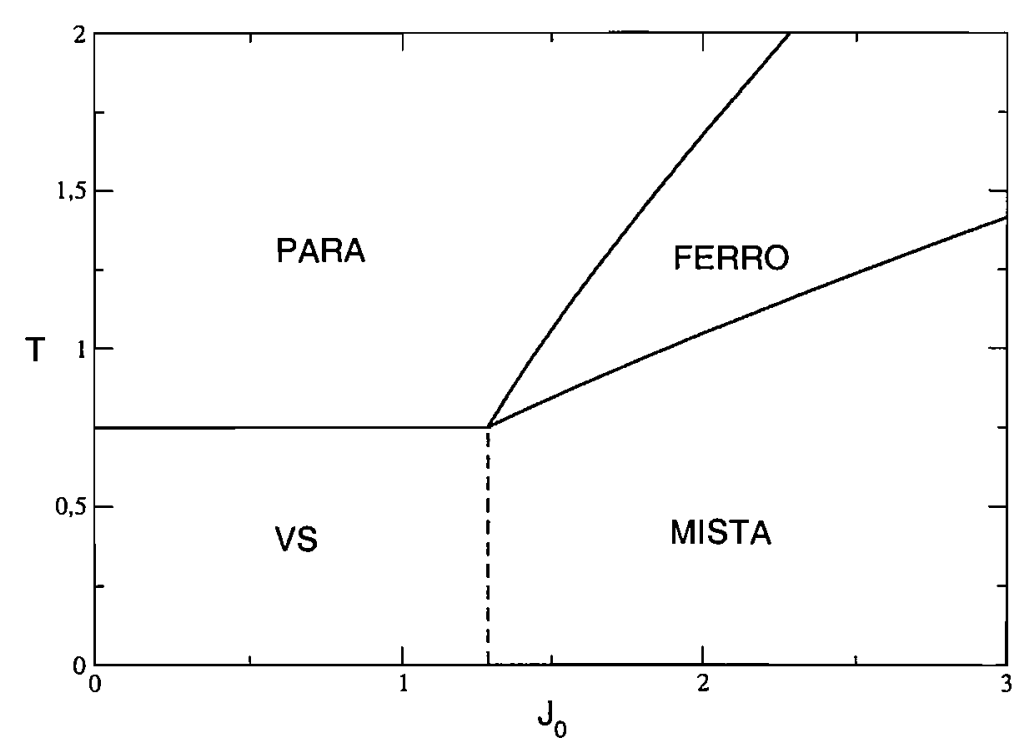

Figura 2.9: diagrama de fases para uma distribuição normal com $\sigma=0,8$ 
Finalmente, o digrama de fases para uma distribuição duplo-delta para $\sigma=1,0$ é mostrado na figura 2.10 .

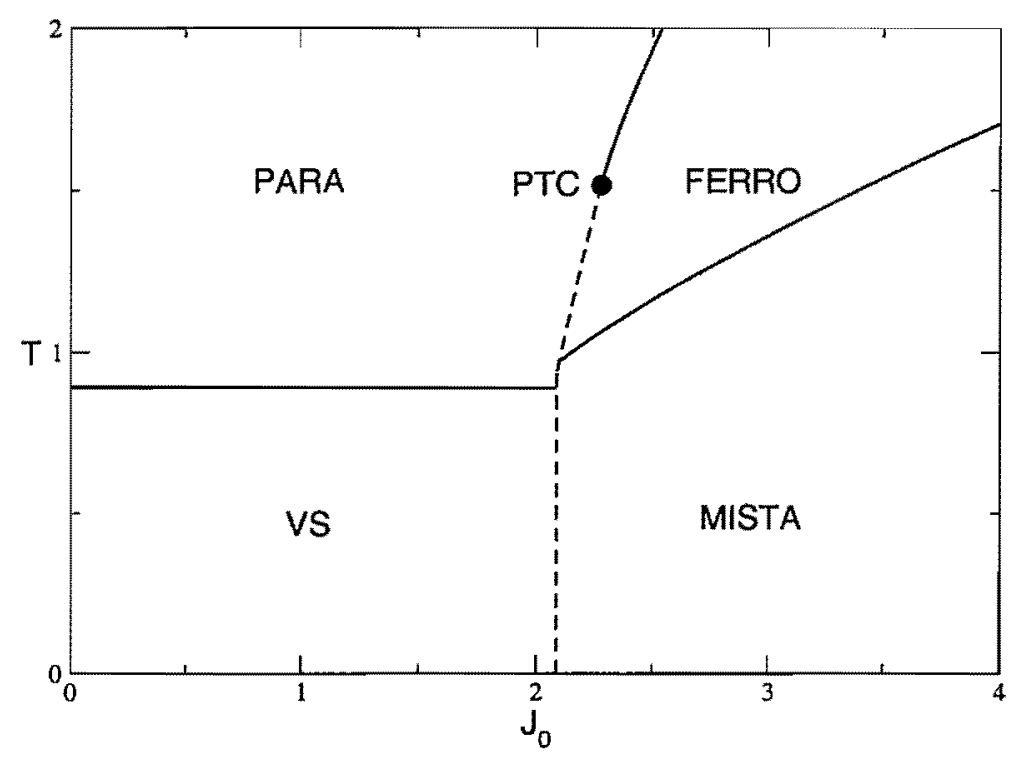

Figura 2.10: diagrama de fases para uma distribuição de campo duplo-delta com $\sigma=1,0$

\subsubsection{Determinação do ponto tricrítico}

Para distribuições de campo aleatórios, $\wp\left(h_{i}\right)$, que decrescem monotonicamente quando aumentamos o $|h|$, tais como a distribuição normal, a transição é sempre contínua. Para distribuições que têm um mínimo em campo zero, tais como a distribuição bimodal (ou duplo-delta), existe um ponto tricrítico sobre a linha crítica e a transição torna-se de primeira ordem para campos grandes. Este resultado foi mostrado por (Salinas e Wreszinski, 1985) para modelos sem a ausência de interação vidro de spin. No caso $H_{0}=0$ e considerando a distribuição duplo-delta acima, podemos determinar o ponto tricrítico expandindo o lado direito da equação (2.134) em potências de $m$

$$
m=a_{1} m+a_{3} m^{3}+a_{5} m^{5}+\cdots
$$


com

$$
\begin{aligned}
& a_{1}=\beta J_{0}\left\langle\operatorname{sech}^{2} \beta H\right\rangle_{H}, \\
& a_{3}=\left(\beta J_{0}\right)^{3}\left[\frac{2}{3}\left\langle\operatorname{sech}^{2} \beta H\right\rangle_{H}-\left\langle\operatorname{sech}^{4} \beta H\right\rangle_{H}\right], \\
& a_{5}=\frac{\left(\beta J_{0}\right)^{5}}{15}\left[2\left\langle\operatorname{sech}^{2} \beta H\right\rangle_{H}-15\left\langle\operatorname{sech}^{4} \beta H\right\rangle_{H}+15\left\langle\operatorname{sech}^{6} \beta H\right\rangle_{H}\right] .
\end{aligned}
$$

O ponto tricrítico é determinado pela seguinte condição para os coeficiente da expansão da magnetização: $a_{1}=1, a_{3}=0$ e $a_{5}>0$. Deste modo obtemos

$$
\beta \sigma=\frac{1}{2} \ln (2+\sqrt{3}), \quad \beta J_{0}=\frac{3}{2}
$$

que nos fornece uma relação analítica entre a temperatura e o valor de $\sigma$ do ponto tricrítico. O valor do desvio padrão $\sigma$ acima do qual surge o ponto tricrítico para a distribuição duplo-delta é dado por

$$
\sigma=\frac{\sqrt{3} \ln (2+\sqrt{3}) J_{0}}{\sqrt{8[3 \ln 6-\sqrt{3} \ln (2+\sqrt{3})]}} \simeq 0,458469 J_{0} .
$$

Para determinar este valor devemos lembrar que o ponto trcrítico surge acima da fase vidro de spin. 


\section{Capítulo 3}

\section{Modelo de energia aleatória no}

\section{ensemble microcanônico}

O modelo de Sherrington e Kirkpatrick (SK) foi proposto para tentar entender a teoria de campo médio para modelos de vidros de spin (Sherrington e Kirkpatrick, 1975). Posteriormente, com a descoberta da instabilidade da solução de SK (Almeida e Thouless, 1978), tornou-se importante conhecer por que o método das réplicas usado por SK era incorreto. Para justificar porque a continuação $n \rightarrow 0$ usada por SK não fornece $\langle\ln Z\rangle_{J}$, uma quebra de simetria no espaço de réplicas foi proposta. Vários trabalhos (Blandin, Gabay, e Garel, 1980; Parisi, 1979) seguindo essa idéia investigam se os cálculos com uma quebra de simetria no espaço de réplicas levariam à expressão verdadeira de $\langle\ln Z\rangle_{J}$. Embora haja um consenso de que o esquema de infinitas quebras de simetria introduzido por Parisi produza uma solução correta para o modelo SK, satisfazendo o critério de estabilidade (Dominicis e Kondor, 1983), e concordando com os experimentos numéricos, não é claro a priori qual o mecanismo responsável por essa forma específica de quebra de simetria.

Um modelo simples de sistema desordenado, o modelo de energias aleatórias (REM), foi introduzido por Derrida para descrever um sistema cujos níveis de energia são variáveis aleatórias independentes (Derrida, 1980). Apesar de sua simplicidade, o modelo captura muitas características de modelos mais complicados como o SK. 
Neste capítulo consideramos uma classe de modelos definidos pela hamiltoniana

$$
\mathcal{H}=-\sum_{1 \leq i_{1}<\cdots<i_{p} \leq N} J_{i_{1} \cdots i_{p}} S_{i_{1}} \cdots S_{i_{p}}+\mathcal{H}_{0}
$$

em que os $N$ spins são do tipo Ising $\left(S_{i}= \pm 1\right)$ e as interações aleatórias $J_{i_{1} \cdots i_{p}}$ são variáveis aleatórias normais independentes com valor esperado 0 e variância $\widetilde{J}^{2}$,

$$
J_{i_{1} \cdots i_{p}} \sim N\left(0, \widetilde{J}^{2}\right)
$$

e $\mathcal{H}_{0}$ é a parte da hamiltoniana independente das interações aleatórias $J_{i_{1} \cdots i_{p}}$; no caso do capítulo anterior, $\mathcal{H}_{0}$ corresponde ao acoplamento ferromagnético entre pares mais o termo de campo aleatório. A variância $\widetilde{J}^{2}$ deve ser escolhida de modo a assegurar a extensividade da energia livre. A forma explícita dessa dependência será determinada de modo que a variância na energia independa de $p$ e coincida com a do modelo SK para $p=2$.

\subsection{Relação com o modelo de energias aleatórias}

Denotemos por $S=\left(S_{1}, \cdots, S_{N}\right)$ uma $2^{N}$ possíveis configurações dos spins no sistema e seja

$$
E_{S}=\mathcal{H}(S)=-\sum_{1 \leq i_{1}<\cdots<i_{p} \leq N} J_{i_{1} \cdots i_{p}} S_{i_{1}} \cdots S_{i_{p}}+\mathcal{H}_{0}(S)
$$

a energia dessa configuração. Essa energia, considerada como função das variáveis aleatórias $J_{i_{1} \cdots i_{p}}$, é uma variável aleatória normal, pois é uma combinação lineare dos $J_{i_{1} \cdots i_{p}}$ (James, 1981).

Os valores esperados das energias para as diferentes configurações dos spins são dados por

$$
\left\langle E_{S}\right\rangle=-\sum_{1 \leq i_{1}<\cdots<i_{p} \leq N}\left\langle J_{i_{1} \cdots i_{p}}\right\rangle S_{i_{1}} \cdots S_{i_{p}}+\mathcal{H}_{0}(S)=\mathcal{H}_{0}(S)=E_{S}^{0}
$$

e a covariância para a energia de duas configurações distintas de spins $S$ e $S^{\prime}$ escolhidas 
entre as $2^{N}$ configurações $S=\left(S_{1}, \cdots, S_{N}\right)$ é dada por

$$
\begin{aligned}
\sigma_{S S^{\prime}} & =\operatorname{Cov}\left(E_{S}, E_{S^{\prime}}\right)=\left\langle\left(E_{S}-E_{S}^{0}\right)\left(E_{S^{\prime}}-E_{S^{\prime}}^{0}\right)\right\rangle \\
& =\sum_{1 \leq i_{1}<\cdots<i_{p} \leq N} \sum_{1 \leq j_{1}<\cdots<j_{p} \leq N}\left\langle J_{i_{1} \cdots i_{p}} J_{j_{1} \cdots j_{p}}\right\rangle S_{i_{1}} \cdots S_{i_{p}} S_{j_{1}}^{\prime} \cdots S_{j_{p}}^{\prime} \\
& =\widetilde{J}^{2} \sum_{1 \leq i_{1}<\cdots<i_{p} \leq N}\left(S_{i_{1}} S_{i_{1}}^{\prime}\right) \cdots\left(S_{i_{p}} S_{i_{p}}^{\prime}\right),
\end{aligned}
$$

em que usamos $\left\langle J_{i_{1} \cdots i_{p}} J_{j_{1} \cdots j_{p}}\right\rangle=\widetilde{J}^{2}$ se todos os índices forem iguais e 0 caso contrário. O símbolo $\langle\cdots\rangle$ indica uma média com relação a distribuição de desordem das variáveis aleatórias das energias $E_{S}$, mas como $E_{S}$ são combinações lineares das variáveis temperadas $J_{i_{1} \cdots i_{p}}$ segue-se o resultado acima.

O somatório nas variáveis de spins, pode ser reescrito como

$$
\left(\sum_{i} S_{i} S_{i}^{\prime}\right)^{p}=p ! \sum_{1 \leq i_{1}<\cdots<i_{p} \leq N}\left(S_{i_{1}} S_{i_{1}}^{\prime}\right) \cdots\left(S_{i_{p}} S_{i_{p}}^{\prime}\right)+O\left(N^{p-1}\right)
$$

Portanto,

$$
\sum_{1 \leq i_{1}<\cdots<i_{p} \leq N}\left(S_{i_{1}} S_{i_{1}}^{\prime}\right) \cdots\left(S_{i_{p}} S_{i_{p}}^{\prime}\right)=\frac{N^{p}}{p !}\left[\left(\frac{1}{N} \sum_{i} S_{i} S_{i}^{\prime}\right)^{p}+O\left(\frac{1}{N}\right)\right] .
$$

Definindo-se a sobreposição (overlap) entre as duas configurações $S$ e $S^{\prime}$ dos spins, segundo

$$
q_{S S^{\prime}}=\frac{1}{N} \sum_{i} S_{i} S_{i}^{\prime}
$$

que é uma medida da "distância" entre as duas configurações, isto é, uma medida do número de spins idênticos nas duas configurações $S$ e $S^{\prime}$, de modo que $-1 \leq q_{S S^{\prime}} \leq 1$. O limite inferior da desigualdade $q_{S S^{\prime}}=-1$ nos diz que as duas configurações de spins são completamente distintas, enquanto o limite superior, $q_{S S^{\prime}}=1$, temos o caso em que as duas configurações são exatamente iguais. A covariância para a energia de duas configurações de spins $S$ e $S^{\prime}$ fica

$$
\sigma_{S S^{\prime}}=\frac{\widetilde{J}^{2} N^{p}}{p !}\left[q_{S S^{\prime}}^{p}+O\left(\frac{1}{N}\right)\right] .
$$

Quando $S=S^{\prime}$, temos $q_{S S}=1$ e a variância na energia é dada por

$$
\operatorname{Var} E_{S}=\sigma_{S S}=\frac{\widetilde{J^{2}} N^{p}}{p !}\left[1+O\left(\frac{1}{N}\right)\right]
$$


No caso do modelo SK, temos $p=2$ e $\widetilde{J}^{2}=J^{2} / N$, de modo que

$$
\operatorname{Var} E_{S}=\frac{J^{2} N}{2}\left[1+O\left(\frac{1}{N}\right)\right]
$$

Assim, para que a variância na energia tenha sempre esse valor independentemente do valor de $p$, devemos escolher

$$
\widetilde{J^{2}}=\frac{p ! J^{2}}{2 N^{p-1}}
$$

Finalmente, a covariância para a energia de duas configurações de spins $S$ e $S^{\prime}$ com a escolha de $\widetilde{J}^{2}$ dada pela eq. (3.12)é

$$
\sigma_{S S^{\prime}}=\frac{J^{2} N}{2}\left[q_{S S^{\prime}}^{p}+O\left(\frac{1}{N}\right)\right] .
$$

No limite termodinâmico $N \rightarrow \infty$, as energias $E_{S}$ têm distribuições normais multivariadas com valores esperados

$$
E_{S}^{0}=\mathcal{H}_{0}(S)
$$

com elementos da matriz de covariância iguais a

$$
\sigma_{S S^{\prime}}=\left(\frac{J^{2} N}{2}\right) q_{S S^{\prime}}^{p}
$$

Portanto, as energias correspondentes a $n$ configurações distintas de spins $S^{(1)}, \cdots, S^{(n)}$ formam um vetor aleatório

$$
\mathrm{E}=\left[\begin{array}{c}
E_{S^{(1)}} \\
\vdots \\
E_{S^{(n)}}
\end{array}\right]
$$

que tem distribuição normal $n$-variada e valor esperado com relação à distribuição de desordem das variáveis aleatórias temperadas dada por

$$
\mathrm{E}_{0}=\left[\begin{array}{c}
E_{S^{(1)}}^{0} \\
\vdots \\
E_{S^{(n)}}^{0}
\end{array}\right]
$$

e matriz de covariância

$$
\Sigma=\left[\begin{array}{cccc}
\sigma_{S^{(1)} S^{(1)}} & \sigma_{S^{(1)} S^{(2)}} & \cdots & \sigma_{S^{(1)} S^{(n)}} \\
\sigma_{S^{(2)} S^{(1)}} & \sigma_{S^{(2)} S^{(2)}} & \cdots & \sigma_{S^{(2)} S^{(n)}} \\
\vdots & \vdots & \ddots & \vdots \\
\sigma_{S^{(n)} S^{(1)}} & \sigma_{S^{(n)} S^{(2)}} & \cdots & \sigma_{S^{(n)} S^{(n)}}
\end{array}\right]
$$


em que os elementos da diagonal $\sigma_{S S}$ nos fornece a variância da variável aleatória e os elementos fora da diagonal $\sigma_{S S^{\prime}}\left(S \neq S^{\prime}\right)$ nos fornece a covariância das variáveis aleatórias $E_{S}$ e $E_{S^{\prime}}$.

$O$ vetor energia aleatória $E$ é uma variável aleatória normal com média $E_{0}$ e matriz de covariância $\Sigma$, em notação usual $\mathrm{E} \sim N\left(\mathrm{E}_{0}, \Sigma\right)$ (James, 1981). A densidade de probabilidade normal $n$-variada é dada por

$$
f(\mathrm{E}=\mathbf{E})=\left(\frac{1}{\sqrt{2 \pi}}\right)^{n} \frac{1}{\sqrt{|\operatorname{det} \Sigma|}} \exp \left[-\frac{1}{2}\left(\widetilde{\mathbf{E}}-\widetilde{\mathrm{E}}_{0}\right) \Sigma^{-1}\left(\mathbf{E}-\mathrm{E}_{0}\right)\right],
$$

em que $\widetilde{M}$ denota a matriz transposta de $M$ e o vetor $\mathbf{E}$ é o valor assumido pelo vetor aleatório $\mathrm{E}$,

$$
\mathbf{E}=\left[\begin{array}{c}
E_{1} \\
\vdots \\
E_{n}
\end{array}\right] .
$$

Para $n=1$, as matrizes reduzem-se a números. Assim

$$
\begin{aligned}
& \mathrm{E}_{0}=E_{S}^{0}, \\
& \Sigma=\sigma_{S S}=N J^{2} / 2,
\end{aligned}
$$

e a densidade de probabilidade é

$$
f\left(E_{S}=E\right)=\frac{1}{\sqrt{\pi N J^{2}}} \exp \left[-\frac{\left(E-E_{S}^{0}\right)^{2}}{N J^{2}}\right]=P\left(E-E_{S}^{0}\right),
$$

que é a distribuição de probabilidade de energia para uma dada configuração de spins $S=\left(S_{1}, \cdots, S_{N}\right)$.

Para $n=2$, o vetor correspondente às energias médias bem como a matriz de covariância para diferentes configurações de spin são dados por

$$
\begin{aligned}
& \mathrm{E}_{0}=\left[\begin{array}{c}
E_{S}^{0} \\
E_{S^{\prime}}^{0}
\end{array}\right], \\
& \Sigma=\left[\begin{array}{ll}
\sigma_{S S} & \sigma_{S S^{\prime}} \\
\sigma_{S^{\prime} S} & \sigma_{S^{\prime} S^{\prime}}
\end{array}\right]=\left(\frac{N J^{2}}{2}\right)\left[\begin{array}{cc}
1 & q^{p} \\
q^{p} & 1
\end{array}\right],
\end{aligned}
$$

em que escrevemos a função sobreposição entre as duas configurações como

$$
q_{S S^{\prime}}=q_{S^{\prime} S}=q .
$$


O determinante da matriz de covariância é

$$
|\operatorname{det} \Sigma|=\left(\frac{N J^{2}}{2}\right)^{2}\left(1-q^{2 p}\right),
$$

com a inversa da matriz de covariância dada por

$$
\Sigma^{-1}=\frac{2}{N J^{2}\left(1-q^{2 p}\right)}\left[\begin{array}{cc}
1 & -q^{p} \\
-q^{p} & 1
\end{array}\right]
$$

Portanto,

$$
\begin{aligned}
& \left(\widetilde{\mathbf{E}}-\widetilde{\mathrm{E}}_{0}\right) \Sigma^{-1}\left(\mathbf{E}-\mathrm{E}_{0}\right)=\frac{2}{N J^{2}\left(1-q^{2 p}\right)}\left[\begin{array}{ll}
E_{1}-E_{S}^{0} & E_{2}-E_{S^{\prime}}^{0}
\end{array}\right]\left[\begin{array}{cc}
1 & -q^{p} \\
-q^{p} & 1
\end{array}\right]\left[\begin{array}{c}
E_{1}-E_{S}^{0} \\
E_{2}-E_{S^{\prime}}^{0}
\end{array}\right] \\
& =\frac{2}{N J^{2}\left(1-q^{2 p}\right)}\left[\left(E_{1}-E_{S}^{0}\right)^{2}-2 q^{p}\left(E_{1}-E_{S}^{0}\right)\left(E_{2}-E_{S^{\prime}}^{0}\right)\right. \\
& \left.+\left(E_{2}-E_{S^{\prime}}^{0}\right)^{2}\right]
\end{aligned}
$$

e a densidade de probabilidade conjunta das energias para as duas configurações de spins $S$ e $S^{\prime}$ fica

$$
\begin{aligned}
f\left(E_{S}=E_{1}, E_{S^{\prime}}=E_{2}\right)= & \frac{1}{\pi N J^{2} \sqrt{1-q^{2 p}}} \\
& \times \exp \left[-\frac{\left(E_{1}-E_{S}^{0}\right)^{2}-2 q^{p}\left(E_{1}-E_{S}^{0}\right)\left(E_{2}-E_{S^{\prime}}^{0}\right)+\left(E_{2}-E_{S^{\prime}}^{0}\right)^{2}}{N J^{2}\left(1-q^{2 p}\right)}\right] .
\end{aligned}
$$

Utilizando a identidade

$$
A^{2}-2 q^{p} A B+B^{2}=\frac{1}{2}\left[(A+B)^{2}\left(1-q^{p}\right)+(A-B)^{2}\left(1+q^{p}\right)\right],
$$

podemos reescrever a densidade de probabilidade conjunta como

$$
\begin{aligned}
f\left(E_{S}=E_{1}, E_{S^{\prime}}=E_{2}\right)= & \frac{1}{\sqrt{\pi N J^{2}\left(1+q^{p}\right) / 2}} \exp \left\{-\frac{\left[\left(E_{1}-E_{S}^{0}\right)+\left(E_{2}-E_{S^{\prime}}^{0}\right)\right]^{2}}{2 N J^{2}\left(1+q^{p}\right)}\right\} \\
& \times \frac{1}{\sqrt{2 \pi N J^{2}\left(1-q^{p}\right)}} \exp \left\{-\frac{\left[\left(E_{1}-E_{S}^{0}\right)-\left(E_{2}-E_{S^{\prime}}^{0}\right)\right]^{2}}{2 N J^{2}\left(1-q^{p}\right)}\right\} .
\end{aligned}
$$

que é exatamente o resultado obtido por Derrida (Derrida, 1980). No limite $q \rightarrow 1$ o último fator na eq.(3.32) torna-se a função delta de Dirac. ${ }^{1}$

\footnotetext{
${ }^{1}$ Utilizamos uma representação para a função delta em termos de uma função gaussiana altamente concentrada no limite $q \rightarrow 1$, ou seja

$$
\delta_{\epsilon}(x)=\lim _{\epsilon \rightarrow \infty} \frac{\epsilon}{\sqrt{\pi}} \exp \left(-\epsilon^{2} x^{2}\right)
$$


Portanto, para $S=S^{\prime}$

$$
f\left(E_{S}=E_{1}, E_{S}=E_{2}\right)=f\left(E_{S}=E_{1}\right) \delta\left(E_{2}-E_{1}\right)=P\left(E_{1}-E_{S}^{0}\right) \delta\left(E_{2}-E_{1}\right)
$$

No caso em que as configurações de spins $S$ e $S^{\prime}$ são macroscopicamente distinguíveis, isto é, se $\left|q_{S S^{\prime}}\right|<1$, então no limite $p \rightarrow \infty$ as energias $E_{S}$ e $E_{S^{\prime}}$ tornam-se descorrelacionadas,

$$
\sigma_{S S^{\prime}}=\left(\frac{J^{2} N}{2}\right) q_{S S^{\prime}}^{p} \rightarrow 0, \quad \text { para } \quad p \rightarrow \infty \quad \text { com } \quad\left|q_{S S^{\prime}}\right|<1
$$

Portanto, no limite $p \rightarrow \infty$

$$
f\left(E_{S^{(1)}}=E_{1}, \cdots, E_{S^{(n)}}=E_{n}\right)=\prod_{i=1}^{n} f\left(E_{S^{(i)}}=E_{i}\right)=\prod_{i=1}^{n} P\left(E_{i}-E_{S^{(i)}}^{0}\right)
$$

para qualquer valor de $n$. Ou seja, os níveis de energia tornam-se variáveis aleatórias independentes. Este é um teorema geral em teoria de probabilidade e diz que: Variáveis normais não correlacionadas são estatisticamente independentes (Mathews e Walker, 1973). Chamamos a atenção que o limite $p \rightarrow \infty$ deve sempre ser tomado depois do limite termodinâmico $N \rightarrow \infty$ de modo que $p \ll N$.

Podemos, portanto, definir o modelo de energias aleatórias (REM) com as três propriedades a seguir:

1. O sistema tem $2^{N}$ níveis de energia $E_{i}$.

2. Os níveis de energia $E_{i}$ são variáveis aleatórias.

3. Os níveis de energia são independentes e distribuídas de acordo com

$$
p(E)=\frac{1}{\sqrt{N J^{2} \pi}} e^{-E^{2} / J^{2} N}
$$

A terceira propriedade é específica do REM, levando a uma grande simplificação permitindo a sua solução exata. Isso pode parecer uma aproximação grosseira para modelos mais realistas, pois as correlações entre os níveis de energia são ignoradas. Apesar disso, o REM apresenta comportamento não trivial que pode servir como ponto de partida para o estudo de modelos mais realistas de vidros de spin. 
temos

$$
\langle n(E)\rangle=\int_{E}^{E+\Delta E} P\left(E_{1}-E_{S}^{0}\right) d E_{1}
$$

A variância de $n(E)$ é dada por

$$
\operatorname{Var} n(E)=\left\langle n(E)^{2}\right\rangle-\langle n(E)\rangle^{2}
$$

em que

$$
\left\langle n(E)^{2}\right\rangle=\int_{E}^{E+\Delta E} d E_{1} \int_{E}^{E+\Delta E} d E_{2}\left\langle\Omega\left(E_{1}\right) \Omega\left(E_{2}\right)\right\rangle
$$

Agora,

$$
\begin{aligned}
\left\langle\Omega\left(E_{1}\right) \Omega\left(E_{2}\right)\right\rangle & =\sum_{S} \sum_{S^{\prime}}\left\langle\delta\left(E_{1}-E_{S}\right) \delta\left(E_{2}-E_{S^{\prime}}\right)\right\rangle=\sum_{S} \sum_{S^{\prime}} f\left(E_{S}=E_{1}, E_{S^{\prime}}=E_{2}\right) \\
& =\sum_{S=S^{\prime}} f\left(E_{S}=E_{1}, E_{S}=E_{2}\right)+\sum_{S \neq S^{\prime}} f\left(E_{S}=E_{1}, E_{S^{\prime}}=E_{2}\right) \\
& =\sum_{S} P\left(E_{1}-E_{S}^{0}\right) \delta\left(E_{2}-E_{1}\right)+\sum_{S \neq S^{\prime}} P\left(E_{1}-E_{S}^{0}\right) P\left(E_{2}-E_{S^{\prime}}^{0}\right) \\
& =\left\langle\Omega\left(E_{1}\right)\right\rangle \delta\left(E_{1}-E_{2}\right)+\left\langle\Omega\left(E_{1}\right)\right\rangle\left\langle\Omega\left(E_{2}\right)\right\rangle-\sum_{S} P\left(E_{1}-E_{S}^{0}\right) P\left(E_{2}-E_{S}^{0}\right) .
\end{aligned}
$$

Portanto,

$$
\begin{aligned}
\left\langle n(E)^{2}\right\rangle= & \langle n(E)\rangle+\langle n(E)\rangle^{2}-\sum_{S}\left[\int_{E}^{E+\Delta E} d E_{1} P\left(E_{1}-E_{S}^{0}\right)\right]^{2} \\
& <\langle n(E)\rangle+\langle n(E)\rangle^{2}
\end{aligned}
$$

o que mostra que

$$
\operatorname{Var} n(E)=\left\langle n(E)^{2}\right\rangle-\langle n(E)\rangle^{2}\langle\langle n(E)\rangle
$$

Concluímos que a flutuação relativa em torno do valor médio satisfaz a desigualdade

$$
\frac{\sqrt{\operatorname{Var} n(E)}}{\langle n(E)\rangle}<\frac{1}{\sqrt{\langle n(E)\rangle}}
$$

Esse resultado é uma conseqüência da independência estatística das variáveis aleatórias $E_{S}$.

Como a tolerância $\Delta E$ é pequena, temos como de hábito,

$$
\langle n(E)\rangle=\sum_{S} \int_{E}^{E+\Delta E} d E_{1} P\left(E_{1}-E_{S}^{0}\right) \approx \Delta E \sum_{S} P\left(E-E_{S}^{0}\right) .
$$


Com a ajuda da função delta de Dirac, podemos escrever

$$
\langle n(E)\rangle=\Delta E \int_{-\infty}^{\infty} d E_{0} P\left(E-E_{0}\right) \Omega_{0}\left(E_{0}\right)
$$

em que

$$
\Omega_{0}\left(E_{0}\right)=\sum_{S} \delta\left(E_{0}-E_{S}^{0}\right)
$$

é a densidade de estados do sistema caracterizado pela hamiltoniana $\mathcal{H}_{0}$. Portanto,

$$
\langle n(E)\rangle=\frac{\Delta E}{\sqrt{\pi N J^{2}}} \int_{-\infty}^{\infty} d E_{0} \exp \left[-\frac{\left(E-E_{0}\right)^{2}}{N J^{2}}+\frac{S_{0}\left(E_{0}\right)}{k_{B}}\right]
$$

em que

$$
S_{0}\left(E_{0}\right)=k_{B} \ln \Omega_{0}\left(E_{0}\right)
$$

é a entropia do sistema caracterizado pela hamiltoniana $\mathcal{H}_{0}$. No limite termodinâmico $N \rightarrow \infty$ a integral é dominada pelo máximo do integrando, de modo que

$$
\ln \langle n(E)\rangle=\max _{E_{0}}\left[-\frac{\left(E-E_{0}\right)^{2}}{N J^{2}}+\frac{S_{0}\left(E_{0}\right)}{k_{B}}\right]
$$

A equação determinando o valor de $E_{0}$ é

$$
\frac{2\left(E-E_{0}\right)}{N J^{2}}+\frac{1}{k_{B}} \frac{\partial S_{0}\left(E_{0}\right)}{\partial E_{0}}=0 .
$$

Como

$$
\frac{\partial S_{0}\left(E_{0}\right)}{\partial E_{0}}=\frac{1}{T_{0}\left(E_{0}\right)}
$$

em que $T_{0}\left(E_{0}\right)$ é a temperatura do sistema caracterizado pela hamiltoniana $\mathcal{H}_{0}$, temos ainda

$$
\frac{1}{T_{0}\left(E_{0}\right)}=-\frac{2 k_{B}\left(E-E_{0}\right)}{N J^{2}}
$$

Portanto, para $E_{0}$ que satisfaz a equação acima,

$$
\ln \langle n(E)\rangle=-\frac{\left(E-E_{0}\right)^{2}}{N J^{2}}+\frac{S_{0}\left(E_{0}\right)}{k_{B}} .
$$

Para valores da energia $E$ tais que $\ln \langle n(E)\rangle>0$, o número médio de estados $\langle n(E)\rangle$ é muito grande, e a flutuação relativa em torno dessa média é muito pequena. Portanto, com probabilidade 1 ,

$$
n(E)=\langle n(E)\rangle
$$


e a entropia de uma dada amostra é, com probabilidade 1,

$$
S(E)=k_{B} \ln n(E)=-\frac{k_{B}\left(E-E_{0}\right)^{2}}{N J^{2}}+S_{0}\left(E_{0}\right) \text {. }
$$

Para valores da energia $E$ tais que $\ln \langle n(E)\rangle<0$, o número médio de estados $\langle n(E)\rangle$ é muito menor que 1. Portanto, com probabilidade 1, não existem amostras com essa energia.

A temperatura do sistema é dada por

$$
\frac{1}{T(E)}=\frac{\partial S(E)}{\partial E}=-\frac{2 k_{B}\left(E-E_{0}\right)}{N J^{2}}
$$

o que mostra que

$$
T(E)=T_{0}\left(E_{0}\right)
$$

Portanto, a energia do sistema em função da temperatura é

$$
E(T)=E_{0}(T)-\frac{N J^{2}}{2 k_{B} T}
$$

em que $E_{0}(T)$ é a energia do sistema caracterizado pela hamiltoniana $\mathcal{H}_{0}$. A entropia como função da temperatura é

$$
S(T)=S_{0}(T)-\frac{N J^{2}}{2 k_{B} T^{2}}
$$

em que $S_{0}(T)$ é a entropia do sistema caracterizado pela hamiltoniana $\mathcal{H}_{0}$. Esses resultados são válidos para temperaturas maiores que a temperatura crítica $T_{c}$, determinada pela condição

$$
S\left(T_{c}\right)=S_{0}\left(T_{c}\right)-\frac{N J^{2}}{2 k_{B} T_{c}^{2}}=0 .
$$

Para temperaturas menores que $T_{c}$ o sistema fica congelado no seu estado em $T=T_{c}$.

Observamos que o modelo definido pela hamiltoniana $\mathcal{H}$ é solúvel desde que o modelo definido pela hamiltoniana $\mathcal{H}_{0}$ também o seja.

\subsection{Modelo com campos aleatórios}

Consideremos o caso em que a hamiltoniana $\mathcal{H}_{0}$ descreve um modelo de Ising ferromagnético de alcance infinito (modelo de Curie-Weiss) na presença de um campo aleatório,

$$
\mathcal{H}_{0}=-\frac{J_{0}}{N} \sum_{i<j} S_{i} S_{j}-\sum_{i} H_{i} S_{i}
$$


em que $H_{i}$ são variáveis aleatórias independentes e identicamente distribuídas.

Observando que

$$
\sum_{i<j} S_{i} S_{j}=\frac{1}{2}\left[\left(\sum_{i} S_{i}\right)^{2}-N\right]
$$

podemos reescrever a hamiltoniana sob a forma

$$
\mathcal{H}_{0}=-\frac{J_{0}}{N}\left(\sum_{i} S_{i}\right)^{2}-\sum_{i} H_{i} S_{i}
$$

em que desprezamos o termo constante $J_{0} / 2$.

A função de partição é

$$
Z_{0}=\sum_{S} e^{-\beta \mathcal{H}_{0}}=\sum_{S} \exp \left[\frac{\beta J_{0} N}{2}\left(\frac{1}{N} \sum_{i} S_{i}\right)^{2}+\beta \sum_{i} H_{i} S_{i}\right] .
$$

Usando a identidade gaussiana

$$
e^{\lambda a^{2} / 2}=\sqrt{\frac{\lambda}{2 \pi}} \int_{-\infty}^{\infty} d x e^{-\lambda x^{2} / 2+\lambda a x}
$$

de modo a linearizar o termo quadrático na função de partição, temos que

$$
Z_{0}=\sqrt{\frac{\beta J_{0} N}{2 \pi}} \int_{-\infty}^{\infty} d m e^{-\beta J_{0} N m^{2} / 2} \prod_{i} \sum_{S_{i}= \pm 1} \exp \left[\beta \sum_{i}\left(J_{0} m+H_{i}\right) S_{i}\right] .
$$

Logo, com o termo quadrático linearizado podemos fazer o traço sobre as variáveis de spin, de modo que

$$
Z_{0}=\sqrt{\frac{\beta J_{0} N}{2 \pi}} \int_{-\infty}^{\infty} d m \exp \left\{N\left[-\frac{1}{2} \beta J_{0} m^{2}+\frac{1}{N} \sum_{i} \ln 2 \cosh \beta\left(J_{0} m+H_{i}\right)\right]\right\}
$$

De acordo com a lei dos grande números, no limite termodinâmico $N \rightarrow \infty$, temos com probabilidade 1

$$
\frac{1}{N} \sum_{i} \ln 2 \cosh \beta\left(J_{0} m+H_{i}\right)=\left\langle\ln 2 \cosh \beta\left(J_{0} m+H\right)\right\rangle_{H}
$$

em que $\langle\cdots\rangle_{H}$ denota o valor esperado com relação a distribuição do campo aleatório $H$. Portanto, qualquer que seja a amostra temos com probabilidade 1,

$$
Z_{0}=\sqrt{\frac{\beta J_{0} N}{2 \pi}} \int_{-\infty}^{\infty} d m \exp \left\{N\left[-\frac{1}{2} \beta J_{0} m^{2}+\left\langle\ln 2 \cosh \beta\left(J_{0} m+H\right)\right\rangle_{H}\right]\right\} .
$$

Além disso, no limite termodinâmico a integral é dominada pelo integrando máximo, de modo que

$$
\ln Z_{0}=N \max _{m}\left[-\frac{1}{2} \beta J_{0} m^{2}+\left\langle\ln 2 \cosh \beta\left(J_{0} m+H\right)\right\rangle_{H}\right]
$$


$O$ valor de $m$ que fornece o máximo no expoente da função de partição $Z_{0}$ é a solução da equação

$$
m=\left\langle\tanh \beta\left(J_{0} m+H\right)\right\rangle_{H}
$$

A energia livre é

$$
F_{0}=-\beta^{-1} \ln Z_{0}=N\left[\frac{1}{2} J_{0} m^{2}-\frac{1}{\beta}\left\langle\ln 2 \cosh \beta\left(J_{0} m+H\right)\right\rangle_{H}\right]
$$

e a energia interna é dada por

$$
E_{0}=-\frac{\partial}{\partial \beta} \ln Z_{0}=N\left[-\frac{1}{2} J_{0} m^{2}-\left\langle H \tanh \beta\left(J_{0} m+H\right)\right\rangle_{H}\right]
$$

A entropia é

$$
S_{0}=k_{B} \beta\left(E_{0}-F_{0}\right)=k_{B}\left[-\beta J_{0} m^{2}-\beta\left\langle H \tanh \beta\left(J_{0} m+H\right)\right\rangle_{H}+\left\langle\ln 2 \cosh \beta\left(J_{0} m+H\right)\right\rangle_{H}\right]
$$

De acordo com os resultados da seção anterior, temos

$$
\mathcal{H}=-\sum_{1 \leq i_{1}<\cdots<i_{p} \leq N} J_{i_{i} \cdots i_{p}} S_{i_{1}} \cdots S_{i_{p}}+\mathcal{H}_{0}
$$

a energia é dada por

$$
\frac{E}{N}=\frac{E_{0}}{N}-\frac{\beta J^{2}}{2}=-\frac{\beta J^{2}}{2}-\frac{1}{2} J_{0} m^{2}-\left\langle H \tanh \beta\left(J_{0} m+H\right)\right\rangle_{H} .
$$

A entropia por spin é dada por

$$
\begin{aligned}
\frac{S}{N k_{B}}= & \frac{S_{0}}{N k_{B}}-\frac{(\beta J)^{2}}{4}=-\frac{(\beta J)^{2}}{4}-\beta J_{0} m^{2}-\beta\left\langle H \tanh \beta\left(J_{0} m+H\right)\right\rangle_{H} \\
& +\left\langle\ln 2 \cosh \beta\left(J_{0} m+H\right)\right\rangle_{H}
\end{aligned}
$$

A energia livre por spin é

$$
\frac{F}{N}=-\frac{\beta J^{2}}{4}+\frac{J_{0} m^{2}}{2}-\beta^{-1}\left\langle\ln 2 \cosh \left(J_{0} m+H\right)\right\rangle_{H}
$$

com a magnetização sendo determinada pela equação

$$
m=\left\langle\tanh \beta\left(J_{0} m+H\right)\right\rangle_{H}
$$

Esses resultados são válidos para $\beta<\beta_{c}, \operatorname{com} \beta_{c}$ determinado pela condição

$$
S\left(\beta_{c}\right)=0
$$


Para $\beta>\beta_{c}$ o sistema fica congelado no seu estado em $\beta=\beta_{c}$. Portanto, a energia é dada por

$$
E(\beta)=E\left(\beta_{c}\right)
$$

e a entropia por

$$
S(\beta)=0
$$

Esses resultados mostram a equivalência, no limite $p \rightarrow \infty$, entre a solução com um passo de quebra de simetria entre réplicas no ensemble canônico, e a solução no ensemble microcanônico. Essa equivalência já havia sido mostrada anteriormente por Gross e Mézard sem a presença de um campo aleatório, com o objetivo de testar os métodos utilizados no estudo de vidros de spin (Gross e Mézard, 1984). 


\section{Capítulo 4}

\section{REM antiferromagnético em um}

\section{campo aleatório}

Existe uma grande classe de materiais que exibem uma transição de uma fase com ordem de longo alcance para uma fase vidro de spin. Exemplos experimentais são as ligas (Eu, Sr)S, $(\mathrm{Pd}, \mathrm{Fe})_{x} \mathrm{Mn}_{1-x}$. Tais transições são chamadas de reentrante. Um exemplo típico de um sistema com transição paramagnética-ferromagnética-vidro de spin é a transição de ligas como $\mathrm{Eu}_{x} \mathrm{Sr}_{1-x} \mathrm{~S} \operatorname{com} 0,51 \leq x \leq 0,65$. Em outros sistemas a transição não está bem definida, sendo a transição do ferromagnetismo para os vidros de spin muito difícil de ser determinada experimentalmente. Entretanto, em materiais antiferromagnéticos, como $\mathrm{FeMgCl}_{2}$, a assinatura da transição ou a coexistência das fases pode ser mais facilmente percebida nos experimentos. $\mathrm{O}$ composto $\mathrm{FeCl}_{2}$ é um antiferromagneto puro com forte anisotropia uniaxial. Diluído com cerca de $50 \%$ de $\mathrm{Mg}$ fornece propriedades tanto de antiferromagneto quanto de vidros de spin. Efeitos similares têm sido observados em $\mathrm{Fe}_{x} \mathrm{Mn}_{1-x} \mathrm{TiO}_{3}$ (Yoshizawa, Mitsuda, Aruga, e Ito, 1987).

Desde do estabelecimento da teoria de campo médio para tratamento de modelos de vidros de spin, tem-se buscado descrever características específicas de uma grande variedades de materiais reais com comportamento vidros de spin. Neste capítulo investigamos o efeito de um campo aleatório em modelo de vidros de spin com interações antiferromagnética de alcance infinito entre duas sub-redes. 


\subsection{O Modelo}

Consideramos um sistema com $2 N$ spins de Ising, $S_{i}= \pm 1, i=1,2, \cdots, 2 N$, localizados em sítios de duas sub-redes idênticas. As interações entre os spins são descrita pela hamiltoniana

$$
\begin{aligned}
\mathcal{H}= & -\sum_{1 \leq i_{1}<\cdots<i_{r} \leq N} \sum_{1 \leq j_{1}<\cdots<j_{r} \leq N} J_{i_{1} \cdots i_{r} j_{1} \cdots j_{r}} S_{i_{1}} \cdots S_{i_{r}} S_{j_{1}} \cdots S_{j_{r}}-\sum_{1 \leq i_{1}<\cdots<i_{p} \leq N} J_{i_{1} \cdots i_{p}}^{\prime} S_{i_{1}} \cdots S_{i_{p}} \\
& -\sum_{1 \leq j_{1}<\cdots<j_{p} \leq N} J_{j_{1} \cdots j_{p}}^{\prime} S_{j_{1}} \cdots S_{j_{p}}-\frac{J_{0}^{\prime}}{N} \sum_{1 \leq i_{1}<i_{2} \leq N} S_{i_{1}} S_{i_{2}}-\frac{J_{0}^{\prime}}{N} \sum_{1 \leq j_{1}<j_{2} \leq N} S_{j_{1}} S_{j_{2}} \\
& +\frac{J_{0}}{N} \sum_{i, j} S_{i} S_{j}-\sum_{k=1}^{2 N} H_{k} S_{k},
\end{aligned}
$$

em que $i \in A$ (sub-rede A), $j \in B$ (sub-rede B) e $k=1,2, \cdots, 2 N$ com cada sub-rede possuindo $N$ spins. A primeira soma é feita sob todos os grupos de $r$ spins pertencentes as diferentes sub-redes. A segunda e a terceira soma são feitas em todos os grupos distintos de $p$ spins pertencentes a mesma sub-rede, a quarta e a quinta soma são feitas em todos os pares distintos de spins pertencentes a mesma sub-rede a sexta soma é feita em todos os pares de spins pertencentes as diferentes sub-redes, finalmente a sétima soma é feita em todos os spins nas duas sub-redes. Optaremos inicialmente por resolver o modelo pelo método de réplicas, pois deste modo podemos utilizar as equações obtidas para estudar toda uma família de modelos com interações de multispins.

As variáveis $J_{i_{1} \cdots i_{r} j_{1} \cdots j_{r}}$ e $J_{i_{1} \cdots i_{p}}$ e $J_{j_{1} \cdots j_{p}}$ são variáveis aleatórias normais independentes e identicamente distribuídas,

$$
\begin{aligned}
J_{i_{1} \cdots i_{r} j_{1} \cdots j_{r}} & \sim N\left(0, \widetilde{J}^{2}\right), \\
J_{i_{1} \cdots i_{p}} & \sim N\left(0, \widetilde{J}^{2}\right), \quad \text { e } \quad J_{j_{1} \cdots j_{p}} \sim N\left(0, \widetilde{J}^{\prime}\right) .
\end{aligned}
$$

As interações ferromagnéticas $J_{0}^{\prime}>0$ dentro de cada sub-rede são, por simplicidade, consideradas como tendo a mesma intensidade, O campo magnético aleatório em cada sítio da rede satisfaz a uma distribuição de probabilidades arbitrária, embora os cálculos numéricos apresentados aqui sejam restritos às distribuições de probabilidades normal e duplo-delta. 
As variâncias nas distribuições de probabilidades normais das interações de troca são escolhidas de modo a garantir a extensividade da energia livre

$$
\begin{aligned}
\widetilde{J}^{2} & =\frac{(r !)^{2}}{N^{2 r-1}} J^{2} \\
\widetilde{J}^{\prime} & =\frac{p !}{2 N^{p-1}} J^{2}
\end{aligned}
$$

Seguindo o procedimento padrão para o cálculo da média do logaritmo da função de partição, é introduzido $n$ réplicas $\alpha=1,2, \cdots, n$ do sistema original de modo que a energia livre por spin é dada por

$$
f=\frac{1}{n} f_{n}, \quad \text { com } \quad f_{n}=\lim _{N \rightarrow \infty}\left(-\frac{1}{2 \beta N} \ln \left\langle\left\langle Z^{n}\right\rangle_{J}\right\rangle_{H}\right)
$$

em que $\beta^{-1}=k_{B} T$. Devemos lembrar que o fator $2 N$ na eq. (4.6) é devido aos $N$ spins de cada sub-rede.

Como as interações de troca e os campos aleatórios são variáveis aleatórias independentes, os cálculos de suas médias podem ser feitas separadamente. Inicialmente, faremos a média sobre a desordem das interaçōes de troca, de modo que

$$
\begin{aligned}
\left\langle Z^{n}\right\rangle_{J}= & \exp \left[\frac{N n \beta^{2}}{2}\left(J^{2}+J^{\prime 2}\right)\right] \operatorname{Tr} \exp \left\{\beta \sum_{\alpha} \sum_{k} H_{k} S_{k}^{\alpha}+\frac{\beta J_{0}^{\prime} N}{2} \sum_{\alpha}\left[\left(m_{A}^{\alpha}\right)^{2}+\left(m_{B}^{\alpha}\right)^{2}\right]\right. \\
& \left.-\beta J_{0} N \sum_{\alpha} m_{A}^{\alpha} m_{B}^{\alpha}+\beta^{2} J^{2} N \sum_{\alpha<\beta}\left(q_{A}^{\alpha \beta} q_{B}^{\alpha \beta}\right)^{r}+\frac{\beta^{2} J^{\prime 2}}{2} \sum_{\alpha<\beta}\left[\left(q_{A}^{\alpha \beta}\right)^{p}+\left(q_{B}^{\alpha \beta}\right)^{p}\right]\right\},
\end{aligned}
$$

com as novas variáveis, magnetização e a sobreposição (overlap) em cada sub-rede, definidas como

$$
\begin{aligned}
& m_{X}^{\alpha}=\frac{1}{N} \sum_{i \in X} S_{i}^{\alpha} \\
& q_{X}^{\alpha \beta}=\frac{1}{N} \sum_{i \in X} S_{i}^{\alpha} S_{i}^{\beta}
\end{aligned}
$$

o índice $X$, indicando as sub-redes $A$ ou $B$.

O traço sobre as variáveis de spins na eq.(4.7) pode ser realizado se utilizarmos uma 
representação integral para a função delta de Dirac

$$
\begin{gathered}
\delta\left(m_{X}^{\alpha}-\frac{1}{N} \sum_{i \in X} S_{i}^{\alpha}\right)=\frac{1}{2 \pi i} \int_{-i \infty}^{i \infty} N d \lambda_{X}^{\alpha} \exp \left[-N \lambda_{X}^{\alpha}\left(m_{X}^{\alpha}-\frac{1}{N} \sum_{i \in X} S_{i}^{\alpha}\right)\right] \quad(X=A, B) \\
\delta\left(q_{X}^{\alpha \beta}-\frac{1}{N} \sum_{i \in X} S_{i}^{\alpha} S_{i}^{\beta}\right)=\frac{1}{2 \pi i} \int_{-i \infty}^{i \infty} N d \lambda_{X}^{\alpha \beta} \exp \left[-N \lambda_{X}^{\alpha \beta}\left(q_{X}^{\alpha \beta}-\frac{1}{N} \sum_{i \in X} S_{i}^{\alpha} S_{i}^{\beta}\right)\right] \quad(X=A, B) .
\end{gathered}
$$

Utilizando a lei dos grandes números podemos fazer a média sobre os campos aleatórios.

Deste modo, a função de partição pode ser reescrita como

$$
\begin{aligned}
\left\langle\left\langle Z^{n}\right\rangle_{J}\right\rangle_{H}= & \prod_{\alpha} \int_{-\infty}^{\infty} d m_{A}^{\alpha} \int_{-i \infty}^{i \infty} \frac{N d \lambda_{A}^{\alpha}}{2 \pi i} \prod_{\alpha} \int_{-\infty}^{\infty} d m_{B}^{\alpha} \int_{-i \infty}^{i \infty} \frac{N d \lambda_{B}^{\alpha}}{2 \pi i} \prod_{\alpha<\beta} \int_{-\infty}^{\infty} d q_{A}^{\alpha \beta} \int_{-i \infty}^{i \infty} \frac{N d \lambda_{A}^{\alpha \beta}}{2 \pi i} \\
& \times \prod_{\alpha<\beta} \int_{-\infty}^{\infty} d q_{B}^{\alpha \beta} \int_{-i \infty}^{i \infty} \frac{N d \lambda_{B}^{\alpha \beta}}{2 \pi i} \exp \left(-2 N \beta f_{n}\right),
\end{aligned}
$$

em que

$$
\begin{aligned}
f_{n}= & \phi+\frac{1}{2 \beta} \sum_{\alpha}\left(\lambda_{A}^{\alpha} m_{A}^{\alpha}+\lambda_{B}^{\alpha} m_{B}^{\alpha}\right)+\frac{1}{2 \beta} \sum_{\alpha<\beta}\left(\lambda_{A}^{\alpha \beta} q_{A}^{\alpha \beta}+\lambda_{B}^{\alpha \beta} q_{B}^{\alpha \beta}\right)-\frac{1}{2 \beta} \ln \left\langle\operatorname{Tr} e^{\overline{\mathcal{H}}_{A}}\right\rangle_{H} \\
& -\frac{1}{2 \beta} \ln \left\langle\operatorname{Tr} e^{\overline{\mathcal{H}}_{B}}\right\rangle_{H},
\end{aligned}
$$

com

$$
\begin{aligned}
\phi= & -\frac{\beta n}{4}\left(J^{2}+J^{\prime 2}\right)+\frac{J_{0}^{\prime} n}{N}+\frac{J_{0}}{2} \sum_{\alpha} m_{A}^{\alpha} m_{B}^{\alpha}-\frac{J_{0}^{\prime}}{4} \sum_{\alpha}\left[\left(m_{A}^{\alpha}\right)^{2}+\left(m_{B}^{\alpha}\right)^{2}\right]-\frac{\beta J^{2}}{2} \sum_{\alpha<\beta}\left(q_{A}^{\alpha \beta} q_{B}^{\alpha \beta}\right)^{r} \\
& -\frac{\beta J^{\prime 2}}{4} \sum_{\alpha<\beta}\left[\left(q_{A}^{\alpha \beta}\right)^{p}+\left(q_{B}^{\alpha \beta}\right)^{p}\right],
\end{aligned}
$$

e a hamiltoniana efetiva das sub-redes $A, B$ dada por

$$
\overline{\mathcal{H}}_{X}=\sum_{\alpha}\left(\beta H+\lambda_{X}^{\alpha}\right) S^{\alpha}+\sum_{\alpha<\beta} \lambda_{X}^{\alpha \beta} S^{\alpha} S^{\beta}
$$

$\operatorname{com} X=A, B$.

No limite termodinâmico $N \rightarrow \infty$, a integração da (4.12), na variável $\lambda_{X}^{\alpha}$ e $\lambda_{X}^{\alpha \beta}$, pode ser feita pelo método do ponto de sela, com as equações de ponto de sela dadas por

$$
\begin{aligned}
& \frac{\partial f_{n}}{\partial \lambda_{X}^{\alpha}}=\frac{1}{2 \beta}\left(m_{X}^{\alpha}-\frac{\left\langle\operatorname{Tr} S^{\alpha} e^{\overline{\mathcal{H}}_{X}}\right\rangle}{\left\langle\operatorname{Tr} e^{\left.\overline{\mathcal{H}}_{X}\right\rangle}\right.}\right)=0, \\
& \frac{\partial f_{n}}{\partial \lambda_{X}^{\alpha \beta}}=\frac{1}{2 \beta}\left(q_{X}^{\alpha \beta}-\frac{\left\langle\operatorname{Tr} S^{\alpha} S^{\beta} e^{\overline{\mathcal{H}}_{X}}\right\rangle}{\left\langle\operatorname{Tr} e^{\left.\overline{\mathcal{H}}_{X}\right\rangle}\right.}\right)=0 .
\end{aligned}
$$


Segue que as equações de ponto de sela são

$$
m_{X}^{\alpha}=\frac{\left\langle\operatorname{Tr} S^{\alpha} \exp \overline{\mathcal{H}}_{X}\right\rangle_{H}}{\left\langle\operatorname{Tr} \exp \overline{\mathcal{H}}_{X}\right\rangle_{H}}, \quad q_{X}^{\alpha \beta}=\frac{\left\langle\operatorname{Tr} S^{\alpha} S^{\beta} \exp \overline{\mathcal{H}}_{X}\right\rangle_{H}}{\left\langle\operatorname{Tr} \exp \overline{\mathcal{H}}_{X}\right\rangle_{H}}, \quad \text { com } \quad(X=A, B)
$$

Estas equações nos permitirão determinar a variável $\lambda_{X}^{\alpha}$ e $\lambda_{X}^{\alpha \beta}$ em termos das variáveis $m$ e $q$. As integrações nas variáveis $m_{X}^{\alpha}$ e $q_{X}^{\alpha \beta}$ podem ser feitas, no limite termodinâmico $N \rightarrow \infty$, pelo método de Laplace. A condição para $f_{n}$ ser estacionária, com relação às variáveis $m_{X}^{\alpha}$ e $q_{X}^{\alpha \beta}$, é dada pelas equações

$$
\begin{aligned}
\frac{\partial f_{n}}{\partial m_{X}^{\alpha}} & =\frac{J_{0}}{2} m_{X}^{\alpha}-\frac{J_{0}^{\prime}}{2} m_{X}^{\alpha}+\frac{1}{2 \beta} \lambda_{X}^{\alpha}=0 \\
\frac{\partial f_{n}}{\partial q_{X}^{\alpha \beta}} & =-\frac{\beta J^{2}}{2} r\left(q_{X}^{\alpha \beta}\right)^{r-1}\left(q_{X}^{\alpha \beta}\right)^{r}-\frac{\beta J^{\prime 2}}{4} p\left(q_{X}^{\alpha \beta}\right)^{p-1}+\frac{1}{2 \beta} \lambda_{X}^{\alpha \beta}=0 .
\end{aligned}
$$

Deste modo, temos

$$
\begin{aligned}
\lambda_{X}^{\alpha} & =\beta\left(J_{0}^{\prime} m_{X}^{\alpha}-J_{0} m \frac{\alpha}{X}\right) \\
\lambda_{X}^{\alpha \beta} & =\beta^{2}\left[J^{2} r\left(q_{X}^{\alpha \beta}\right)^{r-1}\left(q_{\bar{X}}^{\alpha \beta}\right)^{r}+\frac{J^{\prime 2}}{2} p\left(q_{X}^{\alpha \beta}\right)^{p-1}\right]
\end{aligned}
$$

em que $\bar{X}$ é a sub-rede complementar a $X$, isto é, se $X=A$, então $\bar{X}=B$, e vice-versa.

Substituindo as equações de ponto estacionário (4.19) e (4.20) na expressão para $f_{n}$ dada por (4.13), temos

$$
\begin{aligned}
f_{n}= & -\frac{\beta n}{4}\left(J^{2}+J^{\prime 2}\right)-\frac{J_{0}}{2} \sum_{\alpha} m_{A}^{\alpha} m_{B}^{\alpha}+\frac{J_{0}^{\prime}}{4} \sum_{\alpha}\left[\left(m_{A}^{\alpha}\right)^{2}+\left(m_{B}^{\alpha}\right)^{2}\right]+\frac{\beta J^{2}}{2}(2 r-1) \sum_{\alpha<\beta}\left(q_{A}^{\alpha \beta} q_{B}^{\alpha \beta}\right)^{r} \\
& +\frac{\beta J^{\prime 2}}{4}(p-1) \sum_{\alpha<\beta}\left[\left(q_{A}^{\alpha \beta}\right)^{p}+\left(q_{B}^{\alpha \beta}\right)^{p}\right]-\frac{1}{2 \beta} \ln \left\langle\operatorname{Tr} e^{\overline{\mathcal{H}}_{A}}\right\rangle_{H}-\frac{1}{2 \beta} \ln \left\langle\operatorname{Tr} e^{\overline{\mathcal{H}}_{B}}\right\rangle_{H},
\end{aligned}
$$

em que desprezamos os termos que se anulam no limite termodinâmico $N \rightarrow \infty$. Analogamente, a hamiltoniana efetiva das sub-redes $A$ e $B$ quando substituímos as equações de ponto estacionário (4.19) e (4.20) é dada por

$$
\overline{\mathcal{H}}_{X}=\sum_{\alpha} \beta\left(H+J_{0}^{\prime} m_{X}^{\alpha}-J_{0} m_{\bar{X}}^{\alpha}\right) S^{\alpha}+\sum_{\alpha<\beta} \beta^{2}\left[J^{2} r\left(q_{X}^{\alpha \beta}\right)^{r-1}\left(q_{X}^{\alpha \beta}\right)^{r}+\frac{J^{\prime 2}}{2} p\left(q_{X}^{\alpha \beta}\right)^{p-1}\right] S^{\alpha} S^{\beta} .
$$

As demais funções termodinâmicas, podem ser determinadas a por meio de $f_{n}$. 
A energia interna por spin é

$$
\begin{aligned}
\therefore u_{n}= & \frac{\partial\left(\beta f_{n}\right)}{\partial \beta}=-\frac{\beta n}{2}\left(J^{2}+J^{\prime 2}\right)+\frac{J_{0}}{2} \sum_{\alpha} m_{A}^{\alpha} m_{B}^{\alpha}-\frac{J_{0}^{\prime}}{4} \sum_{\alpha}\left[\left(m_{A}^{\alpha}\right)^{2}+\left(m_{B}^{\alpha}\right)^{2}\right] \\
& -\beta J^{2} \sum_{\alpha<\beta}\left(q_{A}^{\alpha \beta} q_{B}^{\alpha \beta}\right)^{r}-\frac{\beta J^{\prime 2}}{2} \sum_{\alpha<\beta}\left[\left(q_{A}^{\alpha \beta}\right)^{p}+\left(q_{B}^{\alpha \beta}\right)^{p}\right]-\frac{1}{2} \sum_{\alpha} \frac{\left\langle H \operatorname{Tr} S^{\alpha} e^{\overline{\mathcal{H}}_{A}}\right\rangle_{H}}{\left\langle\operatorname{Tr} e^{\overline{\mathcal{H}}_{A}}\right\rangle_{H}} \\
& -\frac{1}{2} \sum_{\alpha} \frac{\left\langle H \operatorname{Tr} S^{\alpha} e^{\overline{\mathcal{H}}_{B}}\right\rangle_{H}}{\left\langle\operatorname{Tr} e^{\overline{\mathcal{H}}_{B}}\right\rangle_{H}}
\end{aligned}
$$

e a entropia por spin

$$
\begin{aligned}
\frac{s_{n}}{k_{B}}= & \beta\left(u_{n}-f_{n}\right)=-\frac{\beta^{2} n}{4}\left(J^{2}+J^{\prime 2}\right)+\beta J_{0} \sum_{\alpha} m_{A}^{\alpha} m_{B}^{\alpha}-\frac{\beta J_{0}^{\prime}}{2} \sum_{\alpha}\left[\left(m_{A}^{\alpha}\right)^{2}+\left(m_{B}^{\alpha}\right)^{2}\right] \\
& -\frac{\beta^{2} J^{2}}{2}(2 r+1) \sum_{\alpha<\beta}\left(q_{A}^{\alpha \beta} q_{B}^{\alpha \beta}\right)^{r}-\frac{\beta^{2} J^{\prime 2}}{4}(p+1) \sum_{\alpha<\beta}\left[\left(q_{A}^{\alpha \beta}\right)^{p}+\left(q_{B}^{\alpha \beta}\right)^{p}\right] \\
& +\frac{1}{2} \ln \left\langle\operatorname{Tr} e^{\overline{\mathcal{H}}_{A}}\right\rangle_{H}+\frac{1}{2} \ln \left\langle\operatorname{Tr} e^{\overline{\mathcal{H}}_{B}}\right\rangle_{H}-\frac{\beta}{2} \sum_{\alpha}\left[\frac{\left\langle H \operatorname{Tr} S^{\alpha} e^{\overline{\mathcal{H}}_{A}}\right\rangle_{H}}{\left\langle\operatorname{Tr} e^{\overline{\mathcal{H}}_{A}}\right\rangle_{H}}-\frac{\left\langle H \operatorname{Tr} S^{\alpha} e^{\overline{\mathcal{H}}_{B}}\right\rangle_{H}}{\left\langle\operatorname{Tr} e^{\overline{\mathcal{H}}_{B}}\right\rangle_{H}}\right] .
\end{aligned}
$$

\subsection{Solução Réplica Simétrica}

Conforme visto no capítulo 2, para calcular a energia livre é necessário impor alguma estrutura às variáveis $m_{X}^{\alpha}$ e $q_{X}^{\alpha \beta}$. Como é usual na solução via réplica em que construímos réplicas idênticas, a maneira mais simples é supor que os parâmetros de ordem independam dos índices das réplicas, ou seja, adotar a solução réplica simétrica (RS):

$$
m_{X}^{\alpha}=m_{X}, \quad q_{X}^{\alpha \beta}=q_{X} \quad \operatorname{com} \quad X=A, B
$$

Deste modo, as equações de ponto de sela e ponto estacionário são dadas pelas equações de estado

$$
m_{X}=\left\langle\left\langle\tanh H_{X}\right\rangle_{x}\right\rangle_{H}, \quad q_{X}=\left\langle\left\langle\tanh ^{2} H_{X}\right\rangle_{x}\right\rangle_{H} \quad \text { com } \quad X=A, B
$$

em que a hamiltoniana efetiva é dada por

$$
H_{X}=\beta\left[H+J_{0}^{\prime} m_{X}-J_{0} m_{\bar{X}}+\left(J^{2} r q_{X}^{r-1} q_{X}^{r}+\frac{J^{2}}{2} p q_{X}^{p-1}\right)^{1 / 2} x\right]
$$

e $\langle\cdots\rangle_{x}$ denota uma média com relação a distribuição normal padrão $N(0,1)$,

$$
\langle\cdots\rangle_{x}=\int_{-\infty}^{\infty} \frac{d x}{\sqrt{2 \pi}} e^{-x^{2} / 2}(\cdots)
$$


enquanto $\langle\cdots\rangle_{H}$ denota a média com relação a distribuição de probabilidade do campo aleatório.

A energia livre por spin fica

$$
\begin{aligned}
f= & \lim _{n \rightarrow 0} \frac{f_{n}}{n}=-\frac{\beta}{4}\left(J^{2}+J^{2}\right)-\frac{J_{0}}{2} m_{A} m_{B}+\frac{J_{0}^{\prime}}{4}\left(m_{A}^{2}+m_{B}^{2}\right)-\frac{\beta J^{2}}{4}(2 r-1)\left(q_{A} q_{B}\right)^{r} \\
& -\frac{\beta J^{\prime 2}}{8}(p-1)\left(q_{A}^{p}+q_{B}^{p}\right)+\frac{\beta}{4}\left[J^{2} r\left(q_{A} q_{B}\right)^{r-1}\left(q_{A}+q_{B}\right)+\frac{J^{\prime 2} p}{2}\left(q_{A}^{p-1}+q_{B}^{p-1}\right)\right] \\
& -\frac{1}{2 \beta}\left\langle\left\langle\ln 2 \cosh H_{A}\right\rangle_{x}\right\rangle_{H}-\frac{1}{2 \beta}\left\langle\left\langle\ln 2 \cosh H_{B}\right\rangle_{x}\right\rangle_{H} .
\end{aligned}
$$

\subsection{Casos limites}

Nesta seção obteremos os casos limites do modelo de multispins antiferromagnético na presença de um campo aleátorio e recuperaremos os modelos anteriormente investigados como casos particulares, bem como obteremos o REM antiferromagnético na presença de um campo magnético aleatório.

O modelo SK antiferromagnético na presença do campo aleatório (Vieira et al., 2000) corresponde a $r=1$ e $p=2$. Neste caso

$$
\begin{aligned}
f= & -\frac{\beta J^{2}}{4}\left(1-q_{A}\right)\left(1-q_{B}\right)-\frac{\beta J^{\prime 2}}{8}\left[\left(1-q_{A}\right)^{2}+\left(1-q_{B}\right)^{2}\right]-\frac{J_{0}}{2} m_{A} m_{B}+\frac{J_{0}^{\prime}}{4}\left(m_{A}^{2}+m_{B}^{2}\right) \\
& -\frac{1}{2 \beta}\left\langle\left\langle\ln 2 \cosh H_{A}\right\rangle_{x}\right\rangle_{H}-\frac{1}{2 \beta}\left\langle\left\langle\ln 2 \cosh H_{B}\right\rangle_{x}\right\rangle_{H},
\end{aligned}
$$

em que

$$
m_{X}=\left\langle\left\langle\tanh H_{X}\right\rangle_{x}\right\rangle_{H}, \quad q_{X}=\left\langle\left\langle\tanh ^{2} H_{X}\right\rangle_{x}\right\rangle_{H}, \quad \text { com } \quad X=A, B
$$

e a hamiltoniana efetiva de sub-rede é dada por

$$
H_{X}=\beta\left[H+J_{0}^{\prime} m_{X}-J_{0} m_{\bar{X}}+\left(J^{2} q_{\bar{X}}+J^{\prime 2} q_{X}\right)^{1 / 2} x\right]
$$

em que $X=A, B$. É esperado que este modelo seja apropriado para a descrição de campo médio de um vidro de spin antiferromagnético do tipo $\mathrm{Fe}_{x} \mathrm{Mn}_{1-x} \mathrm{TiO}_{3}$ (Vieira et al., 2000).

O modelo REM antiferromagnético na presença do campo aleatório é obtido no limite $r \rightarrow \infty$ e $p \rightarrow \infty$. Neste caso a energia livre por spin é dada por $f=-\frac{\beta\left(J^{\prime 2}+J^{2}\right)}{4}-\frac{J_{0}}{2} m_{A} m_{B}+\frac{J_{0}^{\prime}}{4}\left(m_{A}^{2}+m_{B}^{2}\right)-\frac{1}{2 \beta}\left\langle\ln 2 \cosh H_{A}\right\rangle_{H}-\frac{1}{2 \beta}\left\langle\ln 2 \cosh H_{B}\right\rangle_{H}$, 
com a hamiltoniana de sub-rede dada por

$$
H_{X}=\beta\left(H+J_{0}^{\prime} m_{X}-J_{0} m_{\bar{X}}\right)
$$

e $X=A, B$.

Para $J^{\prime}=J_{0}^{\prime}=0$ e $H$ uniforme, temos

$$
\begin{aligned}
f & =-\frac{\beta J^{2}}{4}-\frac{J_{0}}{2} m_{A} m_{B}-\frac{1}{2 \beta}\left[\ln 2 \cosh \beta\left(H-J_{0} m_{B}\right)+\ln 2 \cosh \beta\left(H-J_{0} m_{A}\right)\right] \\
& =-\frac{\beta J^{2}}{4}-\frac{J_{0}}{2} m_{A} m_{B}-\frac{1}{2 \beta}\left[\ln 4 \cosh \beta\left(H-J_{0} m_{A}\right) \cosh \beta\left(H-J_{0} m_{B}\right)\right],
\end{aligned}
$$

em que

$$
m_{X}=\tanh \left[\beta\left(H-J_{0} m_{\bar{X}}\right)\right], \quad \text { e } \quad X=A, B .
$$

Recuperando, a menos de uma fator de escala $J \rightarrow J / \sqrt{2}$, o modelo de um vidro de spin antiferromagnético com interação $p$ spins na presença de um campo magnético uniforme (Almeida, 1998). Embora esse modelo seja extremamente simples, ele apresenta um diagrama de fases similar ao de um sistema experimental do tipo $\mathrm{Fe}_{0,5} \mathrm{Zn}_{0,5} \mathrm{~F}_{2}$.

\subsection{Distribuições de campo aleatório}

Para distribuições pares de campo aleatórios $P(H)=P(-H)$ podemos ver que

$$
m_{A}=-m_{B}=m, \quad q_{A}=q_{B}=q
$$

Deste modo, as equações de ponto de sela serão dadas por

$$
m=\langle\tanh H\rangle_{H}, \quad q=\left\langle\tanh ^{2} H\right\rangle_{H},
$$

com

$$
H_{X}=\beta\left[H+\left(J_{0}^{\prime}+J_{0}\right) m\right]
$$

As equações que fornecem a energia livre e os parâmetros de ordem, são similares àquelas obtidas para o modelo SK na presença de um campo aleatório, com média $\left(J_{0}^{\prime}+J_{0}\right) / N$ e variância $\left(J^{\prime 2}+J^{2}\right) / N$. Portanto, os diagramas de fases são similares, com a diferença que a fase ferromagnética é substituída pela fase antiferromagnética. O principal efeito de campo aleatório em um vidro de spin antiferromagnético é diminuir a extensão da fase antiferromagnética. Esse resultado já havia sido obtido para uma versão antiferromagnética do modelo SK na presença de uma campo aleatório (Vieira et al., 2000). 


\section{Capítulo 5}

\section{Solução do REM antiferromagnético}

\section{no ensemble microcanônico}

Neste capítulo consideramos novamente o modelo definido pela hamiltoniana

$$
\begin{aligned}
\mathcal{H}= & -\sum_{1 \leq i_{1}<\cdots<i_{r} \leq N} \sum_{1 \leq j_{1}<\cdots<j_{r} \leq N} J_{i_{1} \cdots i_{r} j_{1} \cdots j_{r}} S_{i_{1}} \cdots S_{i_{r}} S_{j_{1}} \cdots S_{j_{r}}-\sum_{1 \leq i_{1}<\cdots<i_{p} \leq N} J_{i_{1} \cdots i_{p}}^{\prime} S_{i_{1}} \cdots S_{i_{p}} \\
& -\sum_{1 \leq j_{1}<\cdots<j_{p} \leq N} J_{j_{1} \cdots j_{p}}^{\prime} S_{j_{1}} \cdots S_{j_{p}}+\mathcal{H}_{0}
\end{aligned}
$$

em que $i \in A$ (sub-rede A), $j \in B$ (sub-rede B), com cada sub-rede possuindo $N$ spins do tipo Ising, $S_{i}= \pm 1$. A primeira soma é feita sob todos os grupos de $r$ spins pertencentes as diferentes sub-redes. A segunda e a terceira soma são feitas em todos os grupos distintos de $p$ spins dentro da mesma sub-rede, e $\mathcal{H}_{0}$ é a parte da hamiltoniana independente das interações aleatórias. $\mathcal{H}_{0}$ corresponde ao acoplamento ferromagnético entre pares dentro das sub-redes mais o termo de interação antiferromagnética entre as sub-redes, bem como o termo de campo aleatório

$$
\mathcal{H}_{0}=-\frac{J_{0}^{\prime}}{N} \sum_{i_{1}<i_{2}} S_{i_{1}} S_{i_{2}}-\frac{J_{0}^{\prime}}{N} \sum_{j_{1}<j_{2}} S_{j_{1}} S_{j_{2}}+\frac{J_{0}}{N} \sum_{i, j} S_{i} S_{j}-\sum_{i} H_{i} S_{i},
$$

As variáveis $J_{i_{1} \cdots i_{r} j_{1} \cdots j_{r}}, J_{i_{1} \cdots i_{p}}$ e $J_{j_{1} \cdots j_{p}}$ são variáveis aleatórias normais independentes 
e identicamente distribuídas com

$$
\begin{aligned}
J_{i_{1} \cdots i_{r} j_{1} \cdots j_{r}} & \sim N\left(0, \widetilde{J}^{2}\right), \\
J_{i_{1} \cdots i_{p}} & \sim N\left(0, \widetilde{J}^{\prime}\right), \quad \text { e } \quad J_{j_{1} \cdots j_{p}} \sim N\left(0, \widetilde{J}^{2}\right) .
\end{aligned}
$$

As variâncias $\widetilde{J}^{2}$ e $\widetilde{J}^{2}$ devem ser escolhidas de modo a assegurar a extensividade da energia livre. A forma explícita dessa dependência já foi determinada no capítulo 4 .

\subsection{Relação com o modelo de energias aleatórias}

Denotemos por $S=\left(S_{1}^{A}, \cdots, S_{N}^{A} ; S_{1}^{B}, \cdots, S_{N}^{B}\right)$ uma das $2^{2 N}$ possíveis configurações de spins. A energia dessa configuração pode ser escrita como

$$
\begin{aligned}
E_{S}= & \mathcal{H}(S)=-\sum_{1 \leq i_{1}<\cdots<i_{r} \leq N} \sum_{1 \leq j_{1}<\cdots<j_{r} \leq N} J_{i_{1} \cdots i_{r} j_{1} \cdots j_{r}} S_{i_{1}} \cdots S_{i_{r}} S_{j_{1}} \cdots S_{j_{r}} \\
& -\sum_{1 \leq i_{1}<\cdots<i_{p} \leq N} J_{i_{1} \cdots i_{p}}^{\prime} S_{i_{1}} \cdots S_{i_{p}}-\sum_{1 \leq j_{1}<\cdots<j_{p} \leq N} J_{j_{1} \cdots j_{p}}^{\prime} S_{j_{1}} \cdots S_{j_{p}}+\mathcal{H}_{0}(S) .
\end{aligned}
$$

Essa energia, assim como no capítulo 3, é uma variável aleatória normal, pois é combinação linear das variáveis aleatórias $J_{i_{1} \cdots i_{r} j_{1} \cdots j_{r}}, J_{i_{1} \cdots i_{p}}^{\prime}$ e $J_{j_{1} \cdots j_{p}}^{\prime}$.

Os valores esperados das energias para as diferentes configurações dos spins são dados por

$$
\begin{aligned}
\left\langle E_{S}\right\rangle= & -\sum_{1 \leq i_{1}<\cdots<i_{r} \leq N} \sum_{1 \leq j_{1}<\cdots<j_{r} \leq N}\left\langle J_{i_{1} \cdots i_{r} j_{1} \cdots j_{r}}\right\rangle S_{i_{1}} \cdots S_{i_{r}} S_{j_{1}} \cdots S_{j_{r}} \\
& -\sum_{1 \leq i_{1}<\cdots<i_{p} \leq N}\left\langle J_{i_{1} \cdots i_{p}}^{\prime}\right\rangle S_{i_{1}} \cdots S_{i_{p}}-\sum_{1 \leq j_{1}<\cdots<j_{p} \leq N}\left\langle J_{j_{1} \cdots j_{p}}^{\prime}\right\rangle S_{j_{1}} \cdots S_{j_{p}}+\mathcal{H}_{0}(S) \\
& =\mathcal{H}_{0}(S)=E_{S}^{0},
\end{aligned}
$$

e a covariância para a energia de duas configurações distintas de spins $S$ e $S^{\prime}$ é dada por

$$
\begin{aligned}
\sigma_{S S^{\prime}}= & \operatorname{Cov}\left(E_{S}, E_{S^{\prime}}\right)=\left\langle\left(E_{S}-E_{S}^{0}\right)\left(E_{S^{\prime}}-E_{S^{\prime}}^{0}\right)\right\rangle \\
= & \widetilde{J}^{2} \sum_{1 \leq i_{1}<\cdots<i_{r} \leq N} \sum_{1 \leq j_{1}<\cdots<j_{r} \leq N}\left(S_{i_{1}} S_{i_{1}}^{\prime}\right) \cdots\left(S_{i_{r}} S_{i_{r}}^{\prime}\right)\left(S_{j_{1}} S_{j_{1}}^{\prime}\right) \cdots\left(S_{j_{r}} S_{j_{r}}^{\prime}\right) \\
& +\widetilde{J}^{2} \sum_{1 \leq i_{1}<\cdots<i_{p} \leq N}\left(S_{i_{1}} S_{i_{1}}^{\prime}\right) \cdots\left(S_{i_{p}} S_{i_{p}}^{\prime}\right)+\widetilde{J}^{\prime} \sum_{1 \leq j_{1}<\cdots<j_{p} \leq N}\left(S_{j_{1}} S_{j_{1}}^{\prime}\right) \cdots\left(S_{j_{p}} S_{j_{p}}^{\prime}\right)
\end{aligned}
$$

em que $\left\langle J_{i_{1} \cdots i_{p}} J_{j_{1} \cdots j_{p}}\right\rangle=\widetilde{J}^{\prime},\left\langle J_{i_{1} \cdots i_{r} j_{1} \cdots j_{r}} J_{k_{1} \cdots k_{r} l_{1} \cdots l_{r}}\right\rangle=\widetilde{J}^{2}$ se todos os índices forem iguais e 0 caso contrário. 
Novamente o somatório nas variáveis de spins, pode ser reescrito usando

$$
\left(\sum_{i} S_{i} S_{i}^{\prime}\right)^{n}=n ! \sum_{1 \leq i_{1}<\cdots<i_{n} \leq N}\left(S_{i_{1}} S_{i_{1}}^{\prime}\right) \cdots\left(S_{i_{n}} S_{i_{n}}^{\prime}\right)+O\left(N^{n-1}\right)
$$

Portanto,

$$
\begin{aligned}
& \sum_{1 \leq i_{1}<\cdots<i_{r} \leq N} \sum_{1 \leq j_{1}<\cdots<j_{r} \leq N}\left(S_{i_{1}} S_{i_{1}}^{\prime}\right) \cdots\left(S_{i_{r}} S_{i_{r}}^{\prime}\right)\left(S_{j_{1}} S_{j_{1}}^{\prime}\right) \cdots\left(S_{j_{r}} S_{j_{r}}^{\prime}\right)=\left[\frac{1}{r !}\left(\sum_{i} S_{i} S_{i}^{\prime}\right)^{r}\right. \\
& \left.+O\left(N^{r-1}\right)\right]\left[\frac{1}{r !}\left(\sum_{j} S_{j} S_{j}^{\prime}\right)^{r}+O\left(N^{r-1}\right)\right]=\frac{N^{2 r}}{(r !)^{2}}\left(\frac{1}{N} \sum_{i \in A} S_{i} S_{i}^{\prime}\right)^{r}\left(\frac{1}{N} \sum_{j \in B} S_{j} S_{j}^{\prime}\right)^{r} \\
& \quad+O\left(N^{2 r-1}\right)
\end{aligned}
$$

e

$$
\sum_{i_{1}<\cdots<i_{p}}\left(S_{i_{1}} S_{i_{1}}^{\prime}\right) \cdots\left(S_{i_{p}} S_{i_{p}}^{\prime}\right)=\frac{N^{p}}{p !}\left[\left(\frac{1}{N} \sum_{i} S_{i} S_{i}^{\prime}\right)^{p}+O\left(\frac{1}{N}\right)\right]
$$

Definimos a sobreposição (overlap) entre as duas configurações $S^{X}$ e $S^{X}$ dos spins em cada sub-rede como

$$
q_{X\left(S S^{\prime}\right)}=\frac{1}{N} \sum_{i} S_{i}^{X} S_{i}^{X}
$$

$\operatorname{com} X=A, B$. A função sobreposição é uma medida da "distância" entre as duas configurações, $S$ e $S^{\prime}$ nas duas sub-redes $A$ e $B$, com $-1 \leq q_{X\left(S S^{\prime}\right)} \leq 1$. O limite inferior da desigualdade $q_{X\left(S S^{\prime}\right)}=-1$ nos diz que as duas configurações de spins são completamente distintas, enquanto no limite superior, $q_{X\left(S S^{\prime}\right)}=1$, as duas configurações são exatamente iguais. A covariância para a energia de duas configurações de spins $S$ e $S^{\prime}$ fica

$$
\sigma_{S S^{\prime}}=\frac{\widetilde{J}^{2} N^{2 r}}{(r !)^{2}}\left[q_{A\left(S S^{\prime}\right)}^{r} q_{B\left(S S^{\prime}\right)}^{r}+O\left(\frac{1}{N}\right)\right]+\frac{\widetilde{J}^{2} N^{p}}{p !}\left[q_{A\left(S S^{\prime}\right)}^{p}+q_{B\left(S S^{\prime}\right)}^{p}+O\left(\frac{1}{N}\right)\right]
$$

Quando $S=S^{\prime}, q_{X(S S)}=1, \operatorname{com} X=A, B$ e a variância na energia é dada por

$$
\operatorname{Var} E_{S}=\sigma_{S S}=\left[\frac{\widetilde{J}^{2} N^{2 r}}{(r !)^{2}}+\frac{2 \widetilde{J}^{2} N^{p}}{p !}\right]\left[1+O\left(\frac{1}{N}\right)\right]
$$

Escolhendo $\widetilde{J}^{2}$ e $\widetilde{J}^{2}$ conforme o capítulo 4, de modo a garantir a extensividade da energia livre,

$$
\begin{aligned}
\widetilde{J}^{2} & =\frac{(r !)^{2}}{N^{2 r-1}} J^{2} \\
\widetilde{J}^{2} & =\frac{p !}{2 N^{p-1}} J^{2}
\end{aligned}
$$


Portanto, segue que a variância é dada por

$$
\operatorname{Var} E_{S}=N\left(J^{2}+J^{\prime 2}\right)+O\left(\frac{1}{N}\right)
$$

Finalmente, temos a covariância para a energia de duas configurações de spins $S$ e $S^{\prime}$ dada por

$$
\sigma_{S S^{\prime}}=N\left\{J^{2}\left[q_{A\left(S S^{\prime}\right)}^{r} q_{B\left(S S^{\prime}\right)}^{r}\right]+\frac{J^{\prime 2}}{2}\left[q_{A\left(S S^{\prime}\right)}^{p}+q_{B\left(S S^{\prime}\right)}^{p}\right]+O\left(\frac{1}{N}\right)\right\} .
$$

No limite termodinâmico $N \rightarrow \infty$, as energias $E_{S}$ são variáveis aleatórias normais multivariadas com valores esperados

$$
E_{S}^{0}=\mathcal{H}_{0}(S)
$$

e a variância e a covariância são dadas por

$$
\begin{aligned}
\sigma_{S S} & =N\left(J^{2}+J^{\prime 2}\right) \\
\sigma_{S S^{\prime}} & =N\left\{J^{2} q_{A\left(S S^{\prime}\right)}^{r} q_{B\left(S S^{\prime}\right)}^{r}+\frac{J^{\prime 2}}{2}\left[q_{A\left(S S^{\prime}\right)}^{p}+q_{B\left(S S^{\prime}\right)}^{p}\right]\right\}
\end{aligned}
$$

Portanto, as energias correspondentes a $n$ configurações distintas de spins $S^{(1)}, \cdots, S^{(n)}$ formam um vetor aleatório

$$
\mathrm{E}=\left[\begin{array}{c}
E_{S^{(1)}} \\
\vdots \\
E_{S^{(n)}}
\end{array}\right],
$$

que tem distribuição normal $n$-variada e valor esperado com relação à distribuição de desordem das variáveis aleatórias temperadas dada por

$$
\mathrm{E}_{0}=\left[\begin{array}{c}
E_{S^{(1)}}^{0} \\
\vdots \\
E_{S^{(n)}}^{0}
\end{array}\right],
$$

e matriz de covariância

$$
\Sigma=\left[\begin{array}{cccc}
\sigma_{S^{(1)} S^{(1)}} & \sigma_{S^{(1)} S^{(2)}} & \cdots & \sigma_{S^{(1)} S^{(n)}} \\
\sigma_{S^{(2)} S^{(1)}} & \sigma_{S^{(2)} S^{(2)}} & \cdots & \sigma_{S^{(2)} S^{(n)}} \\
\vdots & \vdots & \ddots & \vdots \\
\sigma_{S^{(n)} S^{(1)}} & \sigma_{S^{(n)} S^{(2)}} & \cdots & \sigma_{S^{(n)} S^{(n)}}
\end{array}\right]
$$


em que os elementos da diagonal $\sigma_{S S}$ nos fornece a variância da variável aleatória e os elementos fora da diagonal $\sigma_{S S^{\prime}}\left(S \neq S^{\prime}\right)$ nos fornece a covariância das variáveis aleatórias $E_{S}$ e $E_{S^{\prime}}$.

Podemos escrever que o vetor energia aleatória é uma variável aleatória normal com média $E$ e matriz de covariância $\Sigma$ em notação usual $E \sim N\left(E_{0}, \Sigma\right)$. A densidade de probabilidade normal $n$-variada é dada por

$$
f(\mathrm{E}=\mathrm{E})=\left(\frac{1}{\sqrt{2 \pi}}\right)^{n} \frac{1}{\sqrt{|\operatorname{det} \Sigma|}} \exp \left[-\frac{1}{2}\left(\widetilde{\mathbf{E}}-\widetilde{\mathrm{E}}_{0}\right) \Sigma^{-1}\left(\mathbf{E}-\mathrm{E}_{0}\right)\right],
$$

em que $\widetilde{M}$ denota a matriz transposta de $M$ e o vetor $\mathbf{E}$ é o valor assumido pelo vetor aleatório $\mathrm{E}$,

$$
\mathbf{E}=\left[\begin{array}{c}
E_{1} \\
\vdots \\
E_{n}
\end{array}\right]
$$

Para $n=1$, as matrizes reduzem-se a números. Assim

$$
\begin{aligned}
& E_{0}=E_{S}^{0}, \\
& \Sigma=\sigma_{S S}=N\left(J^{2}+J^{\prime 2}\right),
\end{aligned}
$$

e a densidade de probabilidade é

$$
f\left(E_{S}=E\right)=\frac{1}{\sqrt{2 \pi N\left(J^{2}+J^{\prime 2}\right)}} \exp \left[-\frac{\left(E-E_{S}^{0}\right)^{2}}{2 N\left(J^{2}+J^{\prime 2}\right)}\right]=P\left(E-E_{S}^{0}\right)
$$

que é a distribuição de probabilidade de energia para uma dada configuração de spins $S=\left(S_{1}^{A}, \cdots, S_{N}^{A} ; S_{1}^{B}, \cdots, S_{N}^{B}\right)$.

Para $n=2$, o vetor correspondente às energias médias bem como a matriz de covariância para diferentes configurações de spin são dados por

$$
\begin{aligned}
& E_{0}=\left[\begin{array}{c}
E_{S}^{0} \\
E_{S^{\prime}}^{0}
\end{array}\right], \\
& \Sigma=\left[\begin{array}{ll}
\sigma_{S S} & \sigma_{S S^{\prime}} \\
\sigma_{S^{\prime} S} & \sigma_{S^{\prime} S^{\prime}}
\end{array}\right],
\end{aligned}
$$


de modo que

$$
\left(\widetilde{\mathbf{E}}-\widetilde{E}_{0}\right) \Sigma^{-1}\left(\mathbf{E}-E_{0}\right)=\frac{1}{\sigma_{S S}^{2}-\sigma_{S S^{\prime}}^{2}}\left[\begin{array}{ll}
E_{1}-E_{S}^{0} & E_{2}-E_{S^{\prime}}^{0}
\end{array}\right]\left[\begin{array}{cc}
\sigma_{S S} & -\sigma_{S S^{\prime}} \\
-\sigma_{S S^{\prime}} & \sigma_{S S}
\end{array}\right]\left[\begin{array}{c}
E_{1}-E_{S}^{0} \\
E_{2}-E_{S^{\prime}}^{0}
\end{array}\right],
$$

e a densidade de probabilidade conjunta das energias para as duas configurações de spins $S$ e $S^{\prime}$ fica

$$
\begin{aligned}
f\left(E_{S}=E_{1}, E_{S^{\prime}}=E_{2}\right)= & \frac{1}{2 \pi \sqrt{\sigma_{S S}^{2}-\sigma_{S S^{\prime}}^{2}}} \exp \left\{-\frac{1}{2\left(\sigma_{S S}^{2}-\sigma_{S S^{\prime}}\right)}\left[\left(E_{1}-E_{S}^{0}\right)^{2} \sigma_{S S}\right.\right. \\
& \left.\left.-2\left(E_{1}-E_{S}^{0}\right)\left(E_{2}-E_{S^{\prime}}^{0}\right) \sigma_{S S^{\prime}}+\left(E_{2}-E_{S^{\prime}}^{0}\right)^{2} \sigma_{S S}\right]\right\} .
\end{aligned}
$$

Utilizando a identidade

$$
a A^{2}-2 b A B+a B^{2}=\frac{1}{2}\left[(A+B)^{2}(a-b)+(A-B)^{2}(a+b)\right]
$$

a densidade de probabilidade conjunta pode ser reescrita como

$$
\begin{aligned}
f\left(E_{S}=E_{1}, E_{S^{\prime}}=E_{2}\right)= & \frac{1}{\sqrt{\pi\left(\sigma_{S S}+\sigma_{S S^{\prime}}\right)}} \exp \left\{-\frac{\left[\left(E_{1}-E_{S}^{0}\right)+\left(E_{2}-E_{S^{\prime}}^{0}\right)\right]^{2}}{4\left(\sigma_{S S}+\sigma_{S S^{\prime}}\right)}\right\} \\
& \times \frac{1}{\sqrt{4 \pi\left(\sigma_{S S}-\sigma_{S S^{\prime}}\right)}} \exp \left\{-\frac{\left[\left(E_{1}-E_{S}^{0}\right)-\left(E_{2}-E_{S^{\prime}}^{0}\right)\right]^{2}}{4\left(\sigma_{S S}-\sigma_{S S^{\prime}}\right)}\right\} .
\end{aligned}
$$

Para $\sigma_{S S}=N J^{2} / N$ e $\sigma_{S S^{\prime}}=N J^{2} q^{p} / 2$ obtemos exatamente a mesma densidade de probabilidade encontrada no capítulo 3 . Neste caso a densidade de probabilidade é dada por

$$
\begin{aligned}
& f\left(E_{S}=E_{1}, E_{S^{\prime}}=E_{2}\right)=\frac{1}{\sqrt{\pi N\left\{\left[J^{2}\left(1+q_{A}^{r} q_{B}^{r}\right)\right]+J^{\prime 2}\left[1+\frac{1}{2}\left(q_{A}^{p}+q_{B}^{p}\right)\right]\right.}} \\
& \quad \times \exp \left\{-\frac{\left[\left(E_{1}-E_{S}^{0}\right)+\left(E_{2}-E_{S}^{0}\right)\right]^{2}}{4 N\left\{\left[J^{2}\left(1+q_{A}^{r} q_{B}^{r}\right)\right]+J^{2}\left[1+\frac{1}{2}\left(q_{A}^{p}+q_{B}^{p}\right)\right]\right.}\right\} \\
& \quad \times \frac{1}{\left.\sqrt{4 \pi N\left\{\left[J^{2}\left(1-q_{A}^{r} q_{B}^{r}\right)\right]+J^{\prime 2}\left[1-\frac{1}{2}\left(q_{A}^{p}+q_{B}^{p}\right)\right]\right.}\right\}} \\
& \quad \times \exp \left\{-\frac{\left[\left(E_{1}-E_{S}^{0}\right)+\left(E_{2}-E_{S}^{0}\right)\right]^{2}}{4 N\left\{\left[J^{2}\left(1-q_{A}^{r} q_{B}^{r}\right)\right]+J^{2}\left[1-\frac{1}{2}\left(q_{A}^{p}+q_{B}^{p}\right)\right]\right\}}\right\} .
\end{aligned}
$$

Novamente, no caso em que as configurações dos spins são iguais, $S=S^{\prime}$, as funções de sobreposição de cada sub-rede $q_{A}$ e $q_{B}$ serão iguais a 1 e o último termo da equação (5.34) torna-se uma função delta de Dirac como esperado. Portanto,

$$
f\left(E_{S}=E_{1}, E_{S^{\prime}}=E_{2}\right)=f\left(E_{S}=E_{1}\right) \delta\left(E_{2}-E_{1}\right)=P\left(E_{1}-E_{S}^{0}\right) \delta\left(E_{2}-E_{1}\right)
$$


No caso em que as configurações de spins $S$ e $S^{\prime}$ são macroscopicamente distinguíveis, isto é, se $\left|q_{X\left(S S^{\prime}\right)}\right|<1$, as energias $E_{S}$ e $E_{S^{\prime}}$ tornam-se descorrelacionadas no limite $p \rightarrow \infty$ e $r \rightarrow \infty$,

$$
\sigma_{S S^{\prime}} \rightarrow 0, \quad \text { para } \quad p, r \rightarrow \infty \quad \text { com } \quad\left|q_{X\left(S S^{\prime}\right)}\right|<1
$$

com $X=A, B$. Portanto, os níveis de energia tornam-se variáveis aleatórias independentes

$$
f\left(E_{S^{(1)}}=E_{1}, \cdots, E_{S^{(n)}}=E_{n}\right)=\prod_{i=1}^{n} f\left(E_{S^{(i)}}=E_{i}\right)=\prod_{i=1}^{n} P\left(E_{i}-E_{S^{(i)}}^{0}\right)
$$

para qualquer valor de $n$. Esse resultado é completamente análogo ao encontrado no capítulo 3 e nos permitirá resolver também a versão antiferromagnética do REM no ensemble microcanônico.

\subsection{Solução no ensemble microcanônico}

A solução no ensemble microcanônico é análoga ao caso ferromagnético. A única diferença é na distribuição de probabilidade das energias que neste caso é dada por

$$
P\left(E-E_{S}^{0}\right)=\frac{1}{\sqrt{2 \pi N\left(J^{2}+J^{\prime 2}\right)}} \exp \left[-\frac{\left(E-E_{S}^{0}\right)^{2}}{2 N\left(J^{2}+J^{\prime 2}\right)}\right],
$$

com a densidade de estados do sistema dada por

$$
n(E)=\frac{\Delta E}{\sqrt{2 \pi N\left(J^{2}+J^{\prime 2}\right)}} \int_{-\infty}^{\infty} d E_{0} \exp \left[-\frac{\left(E-E_{0}\right)^{2}}{2 N\left(J^{2}+J^{\prime 2}\right)}+\frac{S_{0}\left(E_{0}\right)}{K_{B}}\right],
$$

em que

$$
S_{0}\left(E_{0}\right)=k_{B} \ln \Omega_{0}\left(E_{0}\right)
$$

é a entropia do sistema antiferromagnético caracterizado pela hamiltoniana $\mathcal{H}_{0}$. No limite termodinâmico $N \rightarrow \infty$, a integral é dominada pelo máximo do integrando,

$$
\ln \langle n(E)\rangle=\max _{E_{0}}\left[-\frac{\left(E-E_{0}\right)^{2}}{2 N\left(J^{2}+J^{\prime 2}\right)}+\frac{S_{0}\left(E_{0}\right)}{k_{B}}\right]
$$

com a equação determinando $E_{0}$, dada por

$$
\frac{\left(E-E_{0}\right)}{N\left(J^{2}+J^{\prime 2}\right)}+\frac{1}{k_{B}} \frac{\partial S_{0}\left(E_{0}\right)}{\partial E_{0}}=0
$$


que pode ainda ser escrita como

$$
\frac{\partial S_{0}\left(E_{0}\right)}{\partial E_{0}}=\frac{1}{T_{0}\left(E_{0}\right)}
$$

em que $T_{0}\left(E_{0}\right)$ é a temperatura do sistema caracterizado pela hamiltoniana $\mathcal{H}_{0}$,

$$
\frac{1}{T_{0}\left(E_{0}\right)}=-\frac{k_{B}\left(E-E_{0}\right)}{N\left(J^{2}+J^{\prime 2}\right)}
$$

Portanto, para $E_{0}$ que satisfaz a equação acima,

$$
\ln \langle n(E)\rangle=-\frac{\left(E-E_{0}\right)^{2}}{2 N\left(J^{2}+J^{\prime 2}\right)}+\frac{S_{0}\left(E_{0}\right)}{k_{B}}
$$

Para valores da energia $E$ tais que $\ln \langle n(E)\rangle>0$, o número médio de estados $\langle n(E)\rangle$ é muito grande, e a flutuação relativa em torno dessa média é muito pequena. Portanto, com probabilidade 1 ,

$$
n(E)=\langle n(E)\rangle
$$

e a entropia de uma dada amostra é, com probabilidade 1,

$$
S(E)=k_{B} \ln n(E)=-\frac{k_{B}\left(E-E_{0}\right)^{2}}{2 N\left(J^{2}+J^{\prime 2}\right)}+S_{0}\left(E_{0}\right) .
$$

Para valores da energia $E$ tais que $\ln \langle n(E)\rangle<0$, novamente o número médio de estados $\langle n(E)\rangle$ é muito menor que 1. Portanto, com probabilidade 1, não existem amostras com essa energia.

A temperatura do sistema é dada por

$$
\frac{1}{T(E)}=\frac{\partial S(E)}{\partial E}=-\frac{k_{B}\left(E-E_{0}\right)}{N\left(J^{2}+J^{\prime 2}\right)}
$$

o que mostra que

$$
T(E)=T_{0}\left(E_{0}\right)
$$

Portanto, a energia do sistema em função da temperatura é

$$
E(T)=E_{0}(T)-\frac{N\left(J^{2}+J^{\prime 2}\right)}{k_{B} T}
$$

em que $E_{0}(T)$ é a energia do sistema caracterizado pela hamiltoniana $\mathcal{H}_{0}$. A entropia como função da temperatura é

$$
S(T)=S_{0}(T)-\frac{N\left(J^{2}+J^{\prime 2}\right)}{k_{B} T^{2}},
$$


em que $S_{0}(T)$ é a entropia do sistema caracterizado pela hamiltoniana $\mathcal{H}_{0}$. Esses resultados são válidos para temperaturas maiores que a temperatura crítica $T_{c}$, determinada pela condição

$$
S\left(T_{c}\right)=S_{0}\left(T_{c}\right)-\frac{N\left(J^{2}+J^{2}\right)}{k_{B} T_{c}^{2}}=0 .
$$

Para temperaturas menores que $T_{c}$ a energia livre e todas as demais funções termodinâmicas, magnetização e energia interna, são independente da temperatura. O sistema fica congelado no seu estado em $T=T_{c}$.

Novamente observamos que o modelo definido pela hamiltoniana $\mathcal{H}$ é solúvel desde que o modelo definido pela hamiltoniana $\mathcal{H}_{0}$ o seja.

\subsection{Modelo antiferromagnético em um campo aleatório}

Consideremos o caso de uma hamiltoniana $\mathcal{H}_{0}$ descrevendo um sistema antiferromagnético de alcance infinito na presença de um campo aleatório,

$$
\mathcal{H}_{0}=-\frac{J_{0}^{\prime}}{N} \sum_{i_{1}<i_{2}} S_{i_{1}} S_{i_{2}}-\frac{J_{0}^{\prime}}{N} \sum_{j_{1}<j_{2}} S_{j_{1}} S_{j_{2}}+\frac{J_{0}}{N} \sum_{i, j} S_{i} S_{j}-\sum_{i} H_{i} S_{i},
$$

em que $J_{0}^{\prime}>0$ favorece o acoplamento ferromagnético dentro de cada sub-rede e o acoplamento $J_{0}>0$, entre as sub-redes, favorece o alinhamento antiparalelo dos spins. Os campos externos $H_{i}$ são variáveis aleatórias independentes e identicamente distribuídas.

Observando que

$$
\sum_{i_{1}<i_{2}} S_{i_{1}} S_{i_{2}}=\frac{1}{2}\left[\left(\sum_{i \in X} S_{i}\right)^{2}-N\right]
$$

$\operatorname{com} X=A, B$. Podemos reescrever a hamiltoniana sob a forma

$$
\mathcal{H}_{0}=-\frac{J_{0}^{\prime}}{2 N}\left[\left(\sum_{i \in A} S_{i}\right)^{2}+\left(\sum_{j \in B} S_{j}\right)^{2}\right]+\frac{J_{0}}{N}\left(\sum_{i \in A} S_{i}\right)\left(\sum_{j \in B} S_{i}\right)-\sum_{i} H_{i} S_{i},
$$

em que desprezamos os termos constantes.

A função de partição é

$$
\begin{aligned}
Z_{0}= & \sum_{S} e^{-\beta \mathcal{H}_{0}}=\sum_{S} \exp \left\{\frac{\beta J_{0}^{\prime} N}{2}\left[\left(\frac{1}{N} \sum_{i \in A} S_{i}\right)^{2}+\left(\frac{1}{N} \sum_{j \in B} S_{j}\right)^{2}\right]-\beta J_{0} N\left(\frac{1}{N} \sum_{i \in A} S_{i}\right)\right. \\
& \left.\times\left(\frac{1}{N} \sum_{j \in B} S_{i}\right)+\beta \sum_{i} H_{i} S_{i}\right\}
\end{aligned}
$$


que pode ainda ser escrita com

$$
Z_{0}=\sum_{S} \exp \left[\frac{\beta J_{0}^{\prime} N}{2}\left(m_{A}^{2}+m_{B}^{2}\right)-\beta J_{0} N m_{A} m_{B}+\beta \sum_{k} H_{k} S_{k}\right],
$$

com

$$
m_{X}=\frac{1}{N} \sum_{i \in X} S_{i}, \quad \text { e } \quad X=A, B
$$

O traço sobre as variáveis de spins pode ser realizado usando a identidade

$$
1=\int_{-\infty}^{\infty} d m_{X} \int_{-i \infty}^{i \infty} \frac{N d \lambda_{X}}{2 \pi i} \exp \left[-N \lambda_{X}\left(m_{X}-\frac{1}{N} \sum_{i \in X} S_{i}\right)\right] .
$$

Portanto,

$$
\begin{aligned}
Z_{0}= & \int_{-\infty}^{\infty} d m_{A} \int_{-i \infty}^{i \infty} \frac{N d \lambda_{A}}{2 \pi i} \int_{-\infty}^{\infty} d m_{B} \int_{-i \infty}^{i \infty} \frac{N d \lambda_{B}}{2 \pi i} \exp \left[N \frac{\beta J_{0}^{\prime}}{2}\left(m_{A}^{2}+m_{B}^{2}\right)-N \beta J_{0} m_{A} m_{B}\right. \\
& \left.-N\left(\lambda_{A} m_{A}+\lambda_{B} m_{B}\right)\right] \sum_{S} \exp \left(\sum_{k} \beta H_{k}+\lambda_{A} \sum_{i \in A} S_{i}+\lambda_{B} \sum_{j \in B} S_{j}\right) .
\end{aligned}
$$

Deste modo, temos

$$
Z_{0}=\int_{-\infty}^{\infty} d m_{A} \int_{-i \infty}^{i \infty} \frac{N d \lambda_{A}}{2 \pi i} \int_{-\infty}^{\infty} d m_{B} \int_{-i \infty}^{i \infty} \frac{N d \lambda_{B}}{2 \pi i} \exp (-2 N \beta f)
$$

em que

$$
\begin{aligned}
f\left(m_{A}, m_{B}, \lambda_{A}, \lambda_{B}\right)= & -\frac{J_{0}^{\prime}}{4}\left(m_{A}^{2}+m_{B}^{2}\right)+\frac{J_{0}}{2} m_{A} m_{B}+\frac{1}{2 \beta}\left(\lambda_{A} m_{A}+\lambda_{B} m_{B}\right) \\
& -\frac{1}{2 \beta N} \sum_{i \in A} \ln 2 \cosh \left(\beta H_{i}+\lambda_{A}\right)-\frac{1}{2 \beta N} \sum_{j \in B} \ln 2 \cosh \left(\beta H_{j}+\lambda_{B}\right) .
\end{aligned}
$$

De acordo com a lei dos grande números, no limite termodinâmico $N \rightarrow \infty$ temos com probabilidade 1

$$
\left.\frac{1}{N} \sum_{i} \ln 2 \cosh \left(\beta H_{i}+\lambda_{X}\right)=\left\langle\ln 2 \cosh \beta H+\lambda_{X}\right)\right\rangle_{H},
$$

em que $\langle\cdots\rangle_{H}$ denota o valor esperado com relação a distribuição do campo aleatório $H$.

No limite termodinâmico a integral (5.61) em relação a $\lambda_{A}$ e $\lambda_{B}$ pode ser feita pelo método de ponto de sela

$$
\begin{aligned}
\ln Z_{0}= & -2 \beta N \max _{m}\left[-\frac{J_{0}^{\prime}}{4}\left(m_{A}^{2}+m_{B}^{2}\right)+\frac{J_{0}}{2} m_{A} m_{B}+\frac{1}{2 \beta}\left(\lambda_{A} m_{A}+\lambda_{B} m_{B}\right)\right. \\
& \left.-\frac{1}{2 \beta}\left\langle\ln 2 \cosh \left(\beta H+\lambda_{A}\right)\right\rangle_{H}-\frac{1}{2 \beta}\left\langle\ln 2 \cosh \left(\beta H+\lambda_{B}\right)\right\rangle_{H}\right] .
\end{aligned}
$$


As equações de ponto de sela são dadas por

$$
\frac{\partial f}{\partial \lambda_{X}}=\frac{1}{2 \beta}\left[m_{X}-\left\langle\tanh \left(\beta H+\lambda_{X}\right)\right\rangle_{H}\right] .
$$

Logo

$$
m_{X}=\left\langle\tanh \left(\beta H+\lambda_{X}\right)\right\rangle_{H}
$$

A integração nas variáveis $m_{A}$ e $m_{B}$ é feita pelo método de Laplace com as equações de pontos estacionários dadas por

$$
\lambda_{X}=\beta\left(J_{0}^{\prime} m_{X}-J_{0} m_{\bar{X}}\right)
$$

Portanto, a energia livre fica

$$
\begin{aligned}
\frac{F_{0}}{2 N}= & \frac{J_{0}^{\prime}}{4}\left(m_{A}^{2}+m_{B}^{2}\right)-\frac{J_{0}}{2} m_{A} m_{B}-\frac{1}{2 \beta}\left\langle\ln 2 \cosh \beta\left(H+J_{0}^{\prime} m_{A}-J_{0} m_{B}\right)\right\rangle_{H} \\
& -\frac{1}{2 \beta}\left\langle\ln 2 \cosh \beta\left(H+J_{0}^{\prime} m_{B}-J_{0} m_{A}\right)\right\rangle_{H} .
\end{aligned}
$$

A energia interna é

$$
\begin{aligned}
\frac{E_{0}}{2 N}= & -\frac{J_{0}^{\prime}}{4}\left(m_{A}^{2}+m_{B}^{2}\right)+\frac{J_{0}}{2} m_{A} m_{B}-\frac{1}{2}\left\langle H \tanh \beta\left(H+J_{0}^{\prime} m_{A}-J_{0} m_{B}\right)\right\rangle_{H} \\
& -\frac{1}{2}\left\langle H \tanh \beta\left(H+J_{0}^{\prime} m_{B}-J_{0} m_{A}\right)\right\rangle_{H} .
\end{aligned}
$$

A entropia é

$$
\begin{aligned}
\frac{S_{0}}{2 N k_{B}}= & \frac{\beta}{2 N}\left(E_{0}-F_{0}\right)=-\frac{\beta J_{0}^{\prime}}{2}\left(m_{A}^{2}+m_{B}^{2}\right)+\beta J_{0} m_{A} m_{B} \\
& -\frac{\beta}{2}\left\langle H \tanh \beta\left(H+J_{0}^{\prime} m_{A}-J_{0} m_{B}\right)\right\rangle_{H}-\frac{\beta}{2}\left\langle H \tanh \beta\left(H+J_{0}^{\prime} m_{B}-J_{0} m_{A}\right)\right\rangle_{H} \\
& +\frac{1}{2}\left\langle\ln 2 \cosh \beta\left(H+J_{0}^{\prime} m_{A}-J_{0} m_{B}\right)\right\rangle_{H}+\frac{1}{2}\left\langle\ln 2 \cosh \beta\left(H+J_{0}^{\prime} m_{B}-J_{0} m_{A}\right)\right\rangle_{H} .
\end{aligned}
$$

Portanto, de acordo com os resultados da seção anterior, a energia do modelo (5.1) é dada por

$$
\begin{aligned}
\frac{E}{2 N}= & \frac{E_{0}}{2 N}-\frac{\beta\left(J^{\prime 2}+J^{2}\right)}{2}=-\frac{\beta\left(J^{\prime 2}+J^{2}\right)}{2}-\frac{J_{0}^{\prime}}{4}\left(m_{A}^{2}+m_{B}^{2}\right)+\frac{J_{0}}{2} m_{A} m_{B} \\
& -\frac{1}{2}\left\langle H \tanh \beta\left(H+J_{0}^{\prime} m_{A}-J_{0} m_{B}\right)\right\rangle_{H}-\frac{1}{2}\left\langle H \tanh \beta\left(H+J_{0}^{\prime} m_{B}-J_{0} m_{A}\right)\right\rangle_{H} .
\end{aligned}
$$


A entropia por spin é dada por

$$
\begin{aligned}
\frac{S}{2 N k_{B}}= & \frac{S_{0}}{2 N k_{B}}-\frac{\beta^{2}\left(J^{2}+J^{2}\right)}{4}=-\frac{\beta^{2}\left(J^{\prime 2}+J^{2}\right)}{4}-\frac{\beta J_{0}^{\prime}}{2}\left(m_{A}^{2}+m_{B}^{2}\right)+\beta J_{0} m_{A} m_{B} \\
& -\frac{\beta}{2}\left\langle H \tanh H_{A}\right\rangle_{H}-\frac{\beta}{2}\left\langle H \tanh H_{B}\right\rangle_{H}+\frac{1}{2}\left\langle\ln 2 \cosh H_{A}\right\rangle+\frac{1}{2}\left\langle\ln 2 \cosh H_{B}\right\rangle
\end{aligned}
$$

A energia livre por spin é dada por

$$
f=-\frac{\beta\left(J^{\prime 2}+J^{2}\right)}{4}-\frac{J_{0}}{2} m_{A} m_{B}+\frac{J_{0}^{\prime}}{4}\left(m_{A}^{2}+m_{B}^{2}\right)-\frac{1}{2 \beta}\left\langle\ln 2 \cosh H_{A}\right\rangle-\frac{1}{2 \beta}\left\langle\ln 2 \cosh H_{B}\right\rangle,
$$

com a hamiltoniana de sub-rede dada por

$$
H_{X}=\beta\left(H+J_{0}^{\prime} m_{X}-J_{0} m_{\bar{X}}\right)
$$

com $X=A, B$ e a magnetização sendo determinada pela equação

$$
m_{X}=\left\langle\tanh H_{X}\right\rangle_{H}
$$

Esse resultado é exatamente o que havia sido encontrado no capítulo 4, porém aqui fica claro que eles são válidos apenas para $\beta<\beta_{c}$, em que $\beta_{c}$ é determinado pela condição

$$
S\left(\beta_{c}\right)=0
$$

Para $\beta>\beta_{c}$ o sistema fica congelado no seu estado fundamental com energia $E(\beta)=$ $E\left(\beta_{c}\right)$, bem como todas as demais funções termodinâmicas ficam congeladas com valores iguais ao assumidos em $\beta_{c}$, enquanto a entropia do sistema é igual a zero. 


\section{Capítulo 6}

\section{Conclusões}

Investigamos modelos de vidros de spin com interações de multispins na presença de um campo aleatório. As interações entre os spins são de alcance infinito e satisfazem a uma distribuição de probabilidade gaussiana, enquanto a distribuição do campo aleatório pode ser qualquer, embora nossa análise numérica tenha sido restrita apenas às distribuições do tipo dupla-delta e gaussiana. Estudamos a influência do campo aleatório sobre o diagrama de fases. Em particular, mostramos que para a distribuição duplo-delta surgem transições de primeira ordem e pontos tricríticos, o que não ocorre para a distribuição gaussiana. Isto não é supreendente, pois foi provado que para o modelo de Ising, com interações de longo alcance, na presença de um campo aleatório gaussiano, não é possível a existência de um ponto tricrítico (Salinas e Wreszinski, 1985). Entretanto, em distribuições que têm um mínimo em campo zero, como por exemplo as bimodais, existe um ponto tricrítico sobre a linha crítica e a transição torna-se de primeira ordem para campos grandes (Aharony, 1978). Além disso, obtivemos uma expressão analítica para determinar o valor exato do ponto tricrítico.

O efeito do campo aleatório é estudado tanto para modelos com uma sub-rede (versão ferromagnética) quanto para duas sub-redes (versão antiferromagnética). Em ambas as versões, tomamos dois procedimentos para resolver o problema: o método de réplicas (ensemble canônico) e o método da contagem de estados (ensemble microcanônico).

No método de réplicas o modelo é resolvido para qualquer valor inteiro $p$ (em que $p$ é o número de spins interagentes). Deste modo, recuperamos o funcional energia livre de 
alguns modelos conhecidos na literatura tanto para versão ferromagnética (Soares et al., 1994; Nogueira et al., 1998), quanto para a versão antiferromagnética (Almeida, 1998; Vieira et al., 2000). No caso ferromagnético utilizamos o esquema de Parisi com um passo de quebra de simetria entre réplicas e em seguida tomamos o limite $p \rightarrow \infty$, a fim de obter uma solução completa para o problema.

No método da contagem de estados estendemos a solução de Derrida na presença de um campo aleatório, o que nos permitiu fazer a contagem de estados, evitando assim o problema da "catástrofe da entropia negativa" presente na solução réplica simétrica. Mostramos que qualquer sistema solúvel sem a interação aleatória de $p$ spins continua solúvel com a presença dessa interação, quando tomamos o limite $p \rightarrow \infty$. O efeito da interação aleatória de $p$ spins é simplesmente acrescentar um caráter vidro de spin ao sistema.

Esperamos que a extensão da solução de Derrida, para o REM na presença de um campo aleatório, possa contribuir para um melhor entendimento de sistemas onde o mecanismos responsável pela ocorrência da fase vidro de spin gere também um campo efetivo aleatório, pois, até onde sabemos, todos os modelos que tratavam o problema de campo aleatório em vidros de spin eram baseados no Ansatz réplica simétrica. Contudo, como queremos estudar o efeito da competição entre vidros de spin e campos aleatórios devemos fazê-lo na região de baixas temperaturas, para que o termo entrópico não venha a controlar o papel das transições. Portanto, devemos dispor de modelos que sejam facilmente solúveis na região de baixas temperaturas, uma vez que é justamente nesta região que a solução réplica simétrica apresenta problemas. Embora o esquema de quebra de simetria entre réplicas proposto por Parisi forneça uma solução correta para o problema, o estudo numérico das equações resultantes não é simples.

Acreditamos que embora o REM seja extremamente simples, ele retem muitas características encontradas em sistemas reais, além de servir como ponto de partida para o estudo da dinâmica em sistemas vítreos. 


\section{Apêndice A}

\section{Cálculo dos elementos da matriz}

\section{hessiana}

Neste apêndice vamos determinar os elementos da matriz hessiana para o modelo de $p$ spins ferromagnético na presença de um campo aleátório. De acordo com os resultados da seção 2.1 o funcional energia livre é dado por

$$
\beta f_{n}\left(m_{\alpha}, q_{\alpha \beta}\right)=-\frac{(\beta J)^{2}}{4}+\frac{(\beta J)^{2}}{2}(p-1) \sum_{\alpha<\beta} q_{\alpha \beta}^{2}+\frac{\beta J_{0}}{2} \sum_{\alpha} m_{\alpha}^{2}-\left\langle\ln \operatorname{Tr} e^{-\beta \overline{\mathcal{H}}}\right\rangle_{H}
$$

em que

$$
\overline{\mathcal{H}}=-\sum_{\alpha}\left(H+J_{0} m_{\alpha}\right) S^{\alpha}-\frac{\beta J^{2}}{2} p \sum_{\alpha<\beta} q_{\alpha \beta}^{p-1} S^{\alpha} S^{\beta}
$$

Devemos determinar as derivadas do funcional energia livre com respeito às variáveis $m_{\alpha}$ e $q_{\alpha \beta}$. Temos,

$$
\begin{aligned}
\frac{\partial\left(\beta f_{n}\right)}{\partial m_{\alpha}}= & \beta J_{0} m_{\alpha}-\beta J_{0}\left\langle\frac{\operatorname{Tr} S^{\alpha} e^{-\beta \overline{\mathcal{H}}}}{\operatorname{Tr} e^{-\beta \overline{\mathcal{H}}}}\right\rangle_{H} \\
\frac{\partial^{2}\left(\beta f_{n}\right)}{\partial m_{\alpha} \partial m_{\beta}}= & \beta J_{0}\left(\delta_{\alpha \beta}-\frac{\partial\left\langle\left\langle S^{\alpha}\right\rangle_{s}\right\rangle_{H}}{\partial m_{\beta}}\right) \\
\frac{\partial^{2}\left(\beta f_{n}\right)}{\partial q_{\alpha \beta} \partial m_{\gamma}}= & -\beta J_{0} \frac{\partial\left\langle\left\langle S^{\gamma}\right\rangle_{s}\right\rangle_{H}}{\partial q_{\alpha \beta}} \\
\frac{\partial\left(\beta f_{n}\right)}{\partial q_{\alpha \beta}}= & \frac{(\beta J)^{2}}{2} p(p-1)\left[q_{\alpha \beta}^{p-1}-q_{\alpha \beta}^{p-2}\left\langle\frac{\operatorname{Tr} S^{\alpha} S^{\beta} e^{-\beta \overline{\mathcal{H}}}}{\left.\left.\operatorname{Tr} e^{-\beta \overline{\mathcal{H}}}\right\rangle_{H}\right]}\right.\right. \\
\frac{\partial^{2}\left(\beta f_{n}\right)}{\partial q_{\alpha \beta} \partial q_{\delta \gamma}}= & \frac{(\beta J)^{2}}{2} p(p-1)\left[(p-1) q_{\alpha \beta}^{p-2} \delta_{\alpha \beta, \delta \gamma}-(p-2) q_{\alpha \beta}^{p-3}\left\langle\left\langle S^{\alpha} S^{\beta}\right\rangle_{s}\right\rangle_{H} \delta_{\alpha \beta, \delta \gamma}\right. \\
& \left.-q_{\alpha \beta}^{p-2} \frac{\partial\left\langle\left\langle S^{\alpha} S^{\beta}\right\rangle_{s}\right\rangle_{H}}{\partial q_{\delta \gamma}}\right]
\end{aligned}
$$


Com o valor esperado

$$
\left\langle\langle\mathcal{O}\rangle_{s}\right\rangle_{H}=\left\langle\frac{\operatorname{Tr} \mathcal{O} e^{-\beta \overline{\mathcal{H}}}}{\operatorname{Tr} e^{-\beta \overline{\mathcal{H}}}}\right\rangle_{H}
$$

Segue-se que a derivada de $\left\langle\langle\mathcal{O}\rangle_{s}\right\rangle_{H}$ com relação a $m_{\alpha}$ é

$$
\frac{\partial\left\langle\langle\mathcal{O}\rangle_{s}\right\rangle_{H}}{\partial m_{\alpha}}=\beta J_{0}\left(\left\langle\left\langle\mathcal{O} S^{\alpha}\right\rangle_{s}\right\rangle_{H}-\left\langle\langle\mathcal{O}\rangle_{s}\left\langle S^{\alpha}\right\rangle_{s}\right\rangle_{H}\right)
$$

de maneira análoga, temos

$$
\frac{\partial\left\langle\langle\mathcal{O}\rangle_{s}\right\rangle_{H}}{\partial q_{\alpha \beta}}=\frac{(\beta J)^{2}}{2} p(p-1) q_{\alpha \beta}^{p-2}\left(\left\langle\left\langle\mathcal{O} S^{\alpha} S^{\beta}\right\rangle_{s}\right\rangle_{H}-\left\langle\langle\mathcal{O}\rangle_{s}\left\langle S^{\alpha} S^{\beta}\right\rangle_{s}\right\rangle_{H}\right)
$$

Usando as equações (A.9) e (A.10), temos que as derivadas segundas de $\beta f_{n}\left(m_{\alpha}, q_{\alpha \beta}\right)$ podem ser escritas como

$$
\begin{aligned}
\frac{\partial^{2}\left(\beta f_{n}\right)}{\partial m_{\alpha} \partial m_{\beta}}= & \beta J_{0}\left[\delta_{\alpha \beta}-\beta J_{0}\left(\left\langle\left\langle S^{\alpha} S^{\beta}\right\rangle_{s}\right\rangle_{H}-\left\langle\left\langle S^{\alpha}\right\rangle_{s}\left\langle S^{\beta}\right\rangle_{s}\right\rangle_{H}\right)\right] \\
\frac{\partial^{2}\left(\beta f_{n}\right)}{\partial q_{\alpha \beta} \partial m_{\gamma}}= & -\frac{\beta J_{0}(\beta J)^{2}}{2} p(p-1) q_{\alpha \beta}^{p-2}\left[\left\langle\left\langle S^{\alpha} S^{\beta} S^{\gamma}\right\rangle_{s}\right\rangle_{H}-\left\langle\left\langle S^{\gamma}\right\rangle_{s}\left\langle S^{\alpha} S^{\beta}\right\rangle_{s}\right\rangle_{H}\right] \\
\frac{\partial^{2}\left(\beta f_{n}\right)}{\partial q_{\alpha \beta} \partial q_{\delta \gamma}}= & \frac{(\beta J)^{2}}{2} p(p-1)\left[(p-1) \delta_{\alpha \beta, \delta \gamma} q_{\alpha \beta}^{p-2}-(p-2) q_{\alpha \beta}^{p-3}\left\langle\left\langle S^{\alpha} S^{\beta}\right\rangle_{s}\right\rangle_{H} \delta_{\alpha \beta, \delta \gamma}\right. \\
& \left.-\frac{(\beta J)^{2}}{2} p(p-1) q_{\alpha \beta}^{p-2} q_{\delta \gamma}^{p-2}\left(\left\langle\left\langle S^{\alpha} S^{\beta} S^{\delta} S^{\gamma}\right\rangle_{s}\right\rangle_{H}-\left\langle\left\langle S^{\alpha} S^{\beta}\right\rangle_{s}\left\langle S^{\delta} S^{\gamma}\right\rangle_{s}\right\rangle_{H}\right)\right]
\end{aligned}
$$

As derivadas são tomadas em $q_{\alpha \beta}=q$ e $m_{\alpha}=m$ (solução réplica simétrica). Neste caso os elementos da matriz hessiana podem tomar sete valores distintos.

$$
\text { O bloco } \frac{\partial^{2}\left(\beta f_{n}\right)}{\partial m_{\alpha} \partial m_{\beta}}
$$

Elementos $\operatorname{com} \alpha=\beta$

$$
\begin{aligned}
\frac{\partial^{2}\left(\beta f_{n}\right)}{\partial m_{\alpha}^{2}} & =\beta J_{0}\left[1-\beta J_{0}\left(\left\langle\left\langle\left(S^{\alpha}\right)^{2}\right\rangle_{s}\right\rangle_{H}-\left\langle\left\langle S^{\alpha}\right\rangle_{s}^{2}\right\rangle_{H}\right)\right] \\
& =\beta J_{0}-\left(\beta J_{0}\right)^{2}\left(1-\left\langle\left\langle S^{\alpha}\right\rangle_{s}^{2}\right\rangle_{H}\right) \equiv G_{\alpha \alpha}=A
\end{aligned}
$$

Elementos com $\alpha \neq \beta$

$$
\begin{aligned}
\frac{\partial^{2}\left(\beta f_{n}\right)}{\partial m_{\alpha} \partial m_{\beta}} & =-\left(\beta J_{0}\right)^{2}\left(\left\langle\left\langle S^{\alpha} S^{\beta}\right\rangle_{s}\right\rangle_{H}-\left\langle\left\langle S^{\alpha}\right\rangle_{s}\left\langle S^{\beta}\right\rangle_{s}\right\rangle_{H}\right) \\
& =-\left(\beta J_{0}\right)^{2}\left(\left\langle\left\langle S^{\alpha} S^{\beta}\right\rangle_{s}\right\rangle_{H}-\left\langle\left\langle S^{\alpha}\right\rangle_{s}^{2}\right\rangle_{H}\right) \equiv G_{\alpha \beta}=B
\end{aligned}
$$


O bloco $\frac{\partial^{2}\left(\beta f_{n}\right)}{\partial q_{\alpha \beta} \partial m_{\gamma}}$

Elementos com 2 índices iguais e um diferente

$$
\begin{aligned}
\frac{\partial^{2}\left(\beta f_{n}\right)}{\partial q_{\alpha \beta} \partial m_{\alpha}}= & -\frac{\left(\beta J_{0}\right)(\beta J)^{2}}{2} p(p-1) q^{p-2}\left(\left\langle\left\langle\left(S^{\alpha}\right)^{2} S^{\beta}\right\rangle_{s}\right\rangle_{H}-\left\langle\left\langle S^{\alpha}\right\rangle_{s}\left\langle S^{\alpha} S^{\beta}\right\rangle_{s}\right\rangle_{H}\right) \\
= & -\frac{\left(\beta J_{0}\right)(\beta J)^{2}}{2} p(p-1) q^{p-2}\left(\left\langle\left\langle S^{\beta}\right\rangle_{s}\right\rangle_{H}-\left\langle\left\langle S^{\alpha}\right\rangle_{s}\left\langle S^{\alpha} S^{\beta}\right\rangle_{s}\right\rangle_{H}\right) \equiv G_{\alpha(\alpha \beta)} \\
& =G_{(\alpha \beta) \alpha}=C .
\end{aligned}
$$

Elementos com todos os índices diferentes

$$
\begin{aligned}
\frac{\partial^{2}\left(\beta f_{n}\right)}{\partial q_{\alpha \beta} \partial m_{\gamma}}= & -\frac{\left(\beta J_{0}\right)(\beta J)^{2}}{2} p(p-1) q^{p-2}\left(\left\langle\left\langle S^{\alpha} S^{\beta} S^{\gamma}\right\rangle_{s}\right\rangle_{H}-\left\langle\left\langle S^{\gamma}\right\rangle_{s}\left\langle S^{\alpha} S^{\beta}\right\rangle_{s}\right\rangle_{H}\right) \\
& \equiv G_{\gamma(\alpha \beta)}=G_{(\alpha \beta) \gamma}=D
\end{aligned}
$$

O bloco $\frac{\partial^{2}\left(\beta f_{n}\right)}{\partial q_{\alpha \beta} \partial q_{\delta \gamma}}$

Elementos com índices iguais entre pares

$$
\begin{aligned}
\frac{\partial^{2}\left(\beta f_{n}\right)}{\partial q_{\alpha \beta} \partial q_{\alpha \beta}}= & \frac{(\beta J)^{2}}{2} p(p-1)\left[(p-1) q^{p-2}-(p-2) q^{p-3}\left\langle\left\langle S^{\alpha} S^{\beta}\right\rangle_{s}\right\rangle_{H}-\frac{(\beta J)^{2}}{2} p(p-1) q^{2(p-2)}\right. \\
& \left.\times\left(\left\langle\left\langle\left(S^{\alpha} S^{\beta}\right)^{2}\right\rangle_{s}\right\rangle_{H}-\left\langle\left\langle S^{\beta} S^{\beta}\right\rangle_{s}^{2}\right\rangle_{H}\right)\right] \\
= & \frac{(\beta J)^{2}}{2} p(p-1) q^{p-2}\left[1-\frac{(\beta J)^{2}}{2} p(p-1) q^{p-2}\left(1-\left\langle\left\langle S^{\alpha} S^{\beta}\right\rangle_{s}^{2}\right\rangle_{H}\right)\right] \equiv G_{(\alpha \beta)(\alpha \beta)} \\
= & P .
\end{aligned}
$$

Elementos com um índice igual entre pares

$$
\begin{aligned}
\frac{\partial^{2}\left(\beta f_{n}\right)}{\partial q_{\alpha \beta} \partial q_{\alpha \gamma}} & =-\frac{(\beta J)^{4}}{4} p^{2}(p-1)^{2} q^{2(p-2)}\left(\left\langle\left\langle\left(S^{\alpha}\right)^{2} S^{\beta} S^{\gamma}\right\rangle_{s}\right\rangle_{H}-\left\langle\left\langle S^{\alpha} S^{\beta}\right\rangle_{s}^{2}\right\rangle_{H}\right) \\
& =-\frac{(\beta J)^{4}}{4} p^{2}(p-1)^{2} q^{2(p-2)}\left(\left\langle\left\langle S^{\beta} S^{\gamma}\right\rangle_{s}\right\rangle_{H}-\left\langle\left\langle S^{\alpha} S^{\beta}\right\rangle_{s}^{2}\right\rangle_{H}\right) \equiv G_{(\alpha \beta)(\alpha \gamma)}=Q
\end{aligned}
$$

Elementos com todos os índices diferentes

$$
\begin{aligned}
\frac{\partial^{2}\left(\beta f_{n}\right)}{\partial q_{\alpha \beta} \partial q_{\delta \gamma}} & =-\frac{(\beta J)^{4}}{4} p^{2}(p-1)^{2} q^{2(p-2)}\left(\left\langle\left\langle S^{\alpha} S^{\beta} S^{\delta} S^{\gamma}\right\rangle_{s}\right\rangle_{H}-\left\langle\left\langle S^{\alpha} S^{\beta}\right\rangle_{s}\left\langle S^{\delta} S^{\gamma}\right\rangle_{s}\right\rangle_{H}\right) \\
& =-\frac{(\beta J)^{4}}{4} p^{2}(p-1)^{2} q^{2(p-2)}\left(\left\langle\left\langle S^{\alpha} S^{\beta} S^{\delta} S^{\gamma}\right\rangle_{s}\right\rangle_{H}-\left\langle\left\langle S^{\alpha} S^{\beta}\right\rangle_{s}^{2}\right\rangle_{H}\right) \equiv G_{(\alpha \beta)(\delta \gamma)}=R
\end{aligned}
$$


com

$$
\begin{aligned}
m & =\left\langle\left\langle S^{\alpha}\right\rangle_{s}\right\rangle_{H}=\left\langle\langle\tanh \bar{H}\rangle_{x}\right\rangle_{H} \\
q & =\left\langle\left\langle S^{\alpha} S^{\beta}\right\rangle_{s}\right\rangle_{H}=\left\langle\left\langle\tanh ^{2} \bar{H}\right\rangle_{x}\right\rangle_{H} \\
t & =\left\langle\left\langle S^{\alpha} S^{\beta} S^{\gamma}\right\rangle_{s}\right\rangle_{H}=\left\langle\left\langle\tanh ^{3} \bar{H}\right\rangle_{x}\right\rangle_{H} \\
r & =\left\langle\left\langle S^{\alpha} S^{\beta} S^{\delta} S^{\gamma}\right\rangle_{s}\right\rangle_{H}=\left\langle\left\langle\tanh ^{4} \bar{H}\right\rangle_{x}\right\rangle_{H}
\end{aligned}
$$

em que $\bar{H}=\beta\left(H+J_{0} m+J \sqrt{\frac{\ell}{2} q^{p-1}} x\right)$. Para $p=2$ recuperamos a matriz hessiana do modelo SK (Almeida e Thouless, 1978).

De posse dos elementos da matriz hessiana podemos determinar seus autovalores e discutir a estabilidade da solução réplica simétrica. 


\section{Apêndice B}

\section{Função de partição do REM}

Neste apêndice, obtemos a energia livre do modelo de energia aleatória.

Para calcular a média de $\ln Z$, podemos usar uma representação integral do logaritmo, utilizando a integral de Frullani:

$$
\langle\ln Z\rangle=\int_{0}^{\infty} \frac{e^{-t}-\left\langle e^{-t Z}\right\rangle}{t} d t
$$

Demonstração:

Seja

$$
\begin{aligned}
f(x) & =\int_{0}^{\infty} \frac{e^{-t}-e^{-t x}}{t} d t \\
f^{\prime}(x) & =\int_{0}^{\infty} \frac{t e^{-t x}}{t} d t=-\left.\frac{e^{-t x}}{x}\right|_{0} ^{\infty}=\frac{1}{x} \\
f(x) & =\ln (x)+c
\end{aligned}
$$

Mas, $f(1)=c=0$. Portanto,

$$
f(x)=\ln x=\int_{0}^{\infty} \frac{e^{-t}-e^{-t x}}{t} d t .
$$

Integrando por partes, temos

$$
\begin{aligned}
& \ln x=\left.\ln t\left(e^{-t}-e^{-t x}\right)\right|_{0} ^{\infty}+\int_{0}^{\infty} d t e^{-t} \ln t-\int_{0}^{\infty} d(t x) e^{-t x} \ln t \\
& \ln x=\int_{0}^{\infty} d t e^{-t} \ln t-\int_{0}^{\infty} d \phi e^{-\phi} \ln t
\end{aligned}
$$

Há somente uma questão, agora, é como encontrar uma expressão explícita de $t$ como uma função de $\phi$, em que $t$ e $\phi$ são relacionados por $e^{-\phi}=\left\langle e^{-t Z}\right\rangle$. 
Como a função de partição é

$$
Z=\sum_{i=1}^{2^{N}} e^{-\beta E_{i}}
$$

e os níveis de energia são variáveis aleatórias com distribuição de probabilidade

$$
\begin{aligned}
\wp\left(E_{i}\right) & =\frac{1}{\sqrt{\pi N J^{2}}} e^{-E_{i}^{2} / J^{2} N}, \quad \mathrm{e} \\
e^{-\phi} & =\left\langle e^{-t Z}\right\rangle, \quad \mathrm{com} \\
\langle\cdots\rangle & =\int_{-\infty}^{\infty} \prod_{i} d E_{i} \wp\left(E_{i}\right)(\cdots) .
\end{aligned}
$$

Segue que

$$
\begin{aligned}
e^{-\phi} & =\int_{-\infty}^{\infty} \prod_{i} d E_{i} \wp\left(E_{i}\right) \exp \left(-t \sum_{i=1}^{2^{N}} e^{-\beta E_{i}}\right) \\
& =\prod_{i=1}^{2^{N}} \frac{1}{\left(\pi N J^{2}\right)^{1 / 2}} \int_{-\infty}^{\infty} d E_{i} e^{-E_{i}^{2} / J^{2} N} e^{-t e^{-\beta E_{i}}} \\
& =\left[\frac{1}{\left(\pi N J^{2}\right)^{1 / 2}} \int_{-\infty}^{\infty} d E \exp \left(-\frac{E^{2}}{J^{2} N}-t e^{-\beta E}\right)\right]^{2 N}
\end{aligned}
$$

Fazendo $y=\frac{E}{\sqrt{J^{2} N}}$, temos

$$
e^{-\phi / 2 N}=\frac{1}{\sqrt{\pi}} \int_{-\infty}^{\infty} d y \exp \left(-y^{2}-t e^{-\lambda y}\right)
$$

$\operatorname{com} \lambda=\sqrt{N} \beta J$.

Comportamento assintótico (quando $\lambda$ é grande) da integral do lado direito da (??). Seja

$$
f(t)=\frac{1}{\sqrt{\pi}} \int_{-\infty}^{\infty} d y \exp \left(-y^{2}-t e^{-\lambda y}\right)
$$

provaremos que: se $-\frac{p \lambda^{2}}{2}<\ln t<-(p-1) \frac{\lambda^{2}}{2}$, onde $p$ é inteiro, então

$$
\begin{aligned}
f(t)= & 1-t e^{\lambda^{2} / 4}+\frac{t}{2} e^{\lambda^{2}}+\cdots+(-1)^{p-2} \frac{t^{p-1}}{(p-1) !} e^{(p-1)^{2} \lambda^{2} / 4} \\
& +\frac{1}{\sqrt{\pi} \lambda} e^{-\ln ^{2} t / \lambda^{2}}\left[\Gamma\left(\frac{2 \ln t}{\lambda^{2}}\right)-\frac{1}{\lambda^{2}} \Gamma^{\prime \prime}\left(\frac{2 \ln t}{\lambda^{2}}\right)+O\left(\frac{1}{\lambda^{4}}\right)\right] .
\end{aligned}
$$

Demonstração:

Seja

$$
f(t)=\frac{1}{\sqrt{\pi}} \int_{-\infty}^{\infty} d y \exp \left(-y^{2}-t e^{-\lambda y}\right)
$$


Fazendo $u=t e^{-\lambda y}$, temos

$$
\begin{aligned}
f(t) & =\frac{1}{\sqrt{\pi}} \int_{0}^{\infty} \frac{d u}{\lambda u} \exp \left[-\frac{1}{\lambda^{2}} \ln ^{2}\left(\frac{u}{t}\right)-u\right] \\
& =\frac{1}{\sqrt{\pi} \lambda} \int_{0}^{\infty} \frac{d u}{u} \exp \left[-\frac{1}{\lambda^{2}}(\ln u-\ln t)^{2}-u\right] \\
& =\frac{1}{\sqrt{\pi} \lambda} \int_{0}^{\infty} d u e^{-\ln ^{2} t \lambda^{2}} e^{-\ln u} \exp \left(-\frac{\ln ^{2} u}{\lambda^{2}}+\frac{2 \ln t \ln u}{\lambda^{2}}-u\right) .
\end{aligned}
$$

Portanto,

$$
f(t)=\frac{1}{\sqrt{\pi} \lambda} e^{-\ln ^{2} t / \lambda^{2}} \int_{0}^{\infty} d u \exp \left[\left(\frac{2 \ln t}{\lambda^{2}}-1\right) \ln u-\frac{\ln ^{2} u}{\lambda^{2}}-u\right] .
$$

A convergência da integral é assegurada pela presença do termo $-\ln ^{2} u$ na exponencial. Todavia, quando $\ln t$ é positivo, este termo não é necessário e podemos expandir $\exp \left(-\ln ^{2} u / \lambda^{2}\right)$ e obter:

$$
I=\int_{0}^{\infty} d u e^{\left(\frac{2 \ln t}{\lambda^{2}}-1\right) \ln u} e^{-\frac{\ln ^{2} u}{\lambda^{2}}} e^{-u}, \quad \text { se } \quad \ln t>0
$$

Façamos a expansão do termo intermediário do integrando.

$$
e^{-\frac{\ln ^{2} u}{\lambda^{2}}}=1-\frac{\ln ^{2} u}{\lambda^{2}}+\frac{\ln ^{4} u}{2 \lambda^{4}}+\cdots+(-1)^{p} \frac{\ln ^{2 p} u}{p ! \lambda^{2 p}}+\cdots
$$

Segue que

$$
f(t)=\frac{e^{-\frac{\ln ^{2} t}{\lambda^{2}}}}{\sqrt{\pi} \lambda}\left[\Gamma\left(\frac{2 \ln t}{\lambda^{2}}\right)-\frac{1}{\lambda^{2}} \Gamma^{\prime \prime}\left(\frac{2 \ln t}{\lambda^{2}}\right)+\cdots+(-1)^{p} \frac{1}{\lambda^{2 p} p !} \Gamma^{2 p}\left(\frac{2 \ln t}{\lambda^{2}}\right)+O\left(\frac{1}{\lambda^{2 p+2}}\right)\right]
$$

em que escrevemos

$$
\Gamma^{n}(x)=\int_{0}^{\infty} d t e^{-t} t^{x-1}(\ln t)^{n}, \quad(x>0) .
$$

Isto prova a fórmula eq. (B.10) quando $p=0$. Vamos considerar o caso $p \leq 1$. Derivando $p$ vezes a eq. (B.9) com respeito a $t$, temos

$$
f^{(p)}(t)=(-1)^{p} e^{\frac{p^{2} \lambda^{2}}{4}} f\left(t e^{p \lambda^{2} / 2}\right)
$$

Demonstração:

$$
\begin{gathered}
f(t)=\frac{1}{\sqrt{\pi}} \int_{-\infty}^{\infty} d y e^{-y^{2}} e^{-t e^{-\lambda y}}, \\
y(t)=e^{-t \alpha}, \\
y^{p}=(-\alpha)^{p} e^{-t \alpha}, \quad \text { onde } \quad \alpha=e^{-\lambda y} .
\end{gathered}
$$


Segue que

$$
\begin{aligned}
\frac{d^{p} f(t)}{d t^{p}} & =\frac{(-1)^{p}}{\sqrt{\pi}} \int_{-\infty}^{\infty} d y e^{-y^{2}}\left(e^{-\lambda y}\right)^{p} e^{-t e^{-\lambda y}} \\
& =\frac{(-1)^{p}}{\sqrt{\pi}} \int_{-\infty}^{\infty} d y \exp \left(-y^{2}-t e^{-t e^{-\lambda y}}-\lambda y p\right) \\
& =\frac{(-1)^{p}}{\sqrt{\pi}} \int_{-\infty}^{\infty} d y \exp \left[-\left(y+\frac{\lambda p}{2}\right)^{2}+\frac{\lambda^{2} p^{2}}{4}-t e^{-\lambda y}\right] .
\end{aligned}
$$

Fazendo a mudança de variável $z=y+\lambda p / 2$, temos

$$
f^{p}(t)=-\frac{(-1)^{p}}{\sqrt{\pi}} e^{\frac{\lambda^{2} p^{2}}{4}} \int_{-\infty}^{\infty} d z \exp \left(-z^{2}-t e^{\lambda^{2} p / 2} e^{-\lambda z}\right)
$$

Mas,

$$
f\left(t e^{\lambda^{2} p / 2}\right)=\frac{1}{\sqrt{\pi}} \int_{-\infty}^{\infty} d y \exp \left(-y^{2}-t e^{\lambda^{2} p / 2} e^{\lambda y}\right)
$$

Portanto,

$$
f^{p}(t)=(-1)^{p} e^{\lambda^{2} p^{2} / 4} f\left(t e^{\lambda^{2} p / 2}\right)
$$

Se $t$ é escolhido tal que: $-\frac{p \lambda^{2}}{2}<\ln t<-[(p-1) / 2] \lambda^{2}$ então, $\ln \left(t e^{p \lambda^{2} / 2}\right)$ é positivo: $\ln t>-p \lambda^{2} / 2$.

Podemos substituir $f\left(t e^{\lambda^{2} p / 2}\right)$ na eq. (B.25) por sua expansão eq. (B.16).

A eq. (B.25) vale para $\ln t>0$, portanto vale também para $\ln \left(t e^{\lambda^{2} p / 2}\right)>0$, com $t \rightarrow t e^{\lambda^{2} p / 2}$.

$$
f^{p}(t)=(-1)^{p} e^{\frac{\lambda^{2} p^{2}}{4}} \exp \left[-\frac{\ln ^{2}\left(t e^{p \lambda^{2} / 2}\right)}{\lambda^{2}}\right]\left[\Gamma\left(\frac{2 \ln t}{\lambda^{2}}+p\right)-\frac{1}{\lambda^{2}} \Gamma^{\prime \prime}\left(\frac{2 \ln t}{\lambda^{2}}+p\right)+O\left(\frac{1}{\lambda^{4}}\right)\right]
$$

A solução geral da equação diferencial eq. (B.26) é

$$
f(t)=\alpha_{0}+\alpha_{1} t+\cdots+\alpha_{p-1} t^{p-1}+\frac{1}{\sqrt{\pi} \lambda} \exp \left(-\frac{\ln ^{2} t}{\lambda^{2}}\right)\left[\Gamma\left(\frac{2 \ln t}{\lambda^{2}}\right)-\frac{1}{\lambda^{2}} \Gamma^{\prime \prime}\left(\frac{2 \ln t}{\lambda^{2}}\right)+O\left(\frac{1}{\lambda^{4}}\right)\right],
$$

onde as constantes $\alpha_{0}, \alpha_{1}, \cdots, \alpha_{p-1}$ são constantes de integração da equação diferencial e não são conhecidas a priori. Podemos determinar essas constantes usando a expansão de Taylor de $f(t)$ em torno de $t=0$. Da eqs. (B.9) e (B.25), temos:

$$
\begin{aligned}
f^{p}(t) & =(-1)^{p} e^{\frac{p^{2} \lambda^{2}}{4} f\left(t e^{p} \lambda^{2} / 4\right)}, \quad(p \geq 1) \\
f^{p}(0) & =(-1)^{p} e^{\frac{p^{2} \lambda^{2}}{4}} f(0) . \quad \text { Mas, } f(0)=1 \text { de eq.(B.25). } \\
f^{p}(0) & =(-1)^{p} e^{\frac{p^{2} \lambda^{2}}{4}} .
\end{aligned}
$$


Então a expansão de Taylor de $f(t)$ é:

$$
f(t)=\sum_{p=0}^{\infty} f^{(p)}(0) \frac{t^{p}}{p !}=1-t e^{\lambda^{2} / 4}+\cdots+(-1)^{p} \frac{t^{p-1}}{(p-1) !} e^{(p-1)^{2} \lambda^{2} / 4}+R_{p}(t)
$$

em que $R_{p}(t)=\frac{t^{p}}{p !} f^{(p)}(\theta t)$ com $0 \leq \theta \leq 1$. Mas, temos ainda que

$$
\left|f^{p}(\theta t)\right| \leq\left|f^{p}(0)\right|=e^{\lambda^{2} p^{2} / 4}
$$

$\mathrm{Na}$ eq. (B.29), o resto é menor que qualquer termo da soma quando $t<e^{-p \lambda^{2} / 2+\lambda^{2} / 4}$. Isto significa que se $\ln t / \lambda^{2}<-p / 2+1 / 4$, então

$$
f(t)=1-e^{\lambda^{2} / 4} t+\cdots+(-1)^{p-1} \frac{e^{(p-1)^{2} \lambda^{2} / 4}}{(p-1) !} t^{p-1}+O\left(e^{\lambda^{2} p^{2} / 4} t^{p}\right)
$$

Comparando os termos das expansões eqs. (B.27) e (B.31) para $-p / 2<\frac{\ln t}{\lambda^{2}}<-\frac{p}{2}+\frac{1}{4}$ e portanto,

$$
\alpha_{i}=\frac{f^{(i)}(0)}{i !} \text { e a expansão eq. (B.10) é provado para: }-\frac{p}{2}<\frac{\ln t}{\lambda^{2}}<-\frac{p-1}{2} .
$$

Da eq. (B.8), podemos escrever $t$ como uma função de $\phi$ : Se

$$
-\frac{\lambda^{2}}{2}<\ln t<0
$$

e

$$
\exp \left(-\frac{\phi}{2^{N}}\right)=1+\Gamma\left(\frac{2 \ln t}{\lambda^{2}}\right) \frac{1}{\sqrt{\pi} \lambda} \exp \left(\frac{\ln ^{2} t}{\lambda^{2}}\right)+\cdots .
$$

Portanto, podemos encontrar lnt como função de

$$
\ln t=-\frac{N J}{T} \sqrt{\ln 2}+\frac{T_{c}}{T}\left\{\frac{1}{2} \ln N+\ln \phi-\ln \left[-\Gamma\left(-\frac{T}{T_{c}}\right)\right]+\frac{1}{2} \ln \pi-\ln \left(\frac{T}{J}\right)\right\}+O(1)
$$

em que $T_{c}=J / 2 \sqrt{\ln 2}$. Usando (B.35), podemos verificar a condição (B.33) que pode ser escrita $T<T_{c}$ e de (B.4)

$$
\langle\ln Z\rangle=\frac{N J}{T} \sqrt{\ln 2}-\frac{T_{c}}{2 T} \ln N+\left(1-\frac{T_{c}}{T}\right) \Gamma^{\prime}(1)-\frac{1 T_{c}}{2 T} \ln (4 \pi \ln 2)+\frac{T_{c}}{T} \ln \left[\Gamma\left(1-\frac{T}{T_{c}}\right)\right]+O(1)
$$

$$
\begin{aligned}
& \text { Se }-p \lambda^{2} / 2<\ln t<-(p-1) \lambda^{2} / 2 \operatorname{com} p \geq 2 \\
& \begin{aligned}
\exp \left(-\frac{\phi}{2^{N}}\right)= & 1-t e^{\lambda^{2} / 4}+\frac{t^{2}}{2 !} e^{\lambda^{2}}+\cdots+(-1) p-1 \frac{t^{p-1}}{(p-1) !} e^{(p-1)^{2} \lambda^{2} / 4}+\frac{1}{\sqrt{\pi} \lambda} \exp \left(-\frac{\ln ^{2} t}{\lambda^{2}}\right) \\
& \times \Gamma\left(\frac{2 \ln t}{\lambda^{2}}\right)+\cdots .
\end{aligned}
\end{aligned}
$$


Calculando novamente $\ln t$ como função de $\phi$

$$
\begin{aligned}
\ln t= & -N \ln 2-\frac{\lambda^{2}}{4}+\ln \phi+Q_{p}\left(\frac{\phi}{2^{N}}, e^{\lambda^{2} / 4}\right)+\frac{1}{\sqrt{\pi} \lambda} \exp \left[-\frac{1}{\lambda^{2}}\left(N \ln 2-\frac{\lambda^{2}}{4}\right)^{2}\right. \\
& \left.+\frac{2}{\lambda^{2}}\left(N \ln 2-\frac{\lambda^{2}}{4}\right) \ln \phi\right] \Gamma\left(-\frac{1}{2}-\frac{2 N \ln 2}{\lambda^{2}}\right)+\cdots
\end{aligned}
$$

em que $Q_{p}\left(\phi / 2^{N}, e^{\lambda^{2} / 4}\right)$ é um polinômio nas variáveis $\phi / 2^{N}$ e $e^{\lambda^{2} / 4}$. Por exemplo

$$
\begin{aligned}
& Q_{2}\left(\frac{\phi}{2^{N}}, e^{\lambda^{2} / 4}\right)=0 . \\
& Q_{3}\left(\frac{\phi}{2^{N}}, e^{\lambda^{2} / 4}\right)=\frac{\phi}{2^{N+1}}\left(e^{\lambda^{2} / 2}-1\right) . \\
& Q_{4}\left(\frac{\phi}{2^{N}}, e^{\lambda^{2} / 4}\right)=\frac{\phi}{2^{N+1}}\left(e^{\lambda^{2} / 2}-1\right)-\frac{1}{24} \frac{\phi^{2}}{2^{2 N}}\left(4 e^{3 \lambda^{2} / 2}-9 e^{\lambda^{2}}+6 e^{\lambda^{2} / 2}-1\right) .
\end{aligned}
$$

Usando equação (B.38), vemos que a condição (B.37) torna-se $\sqrt{2 p-3} T_{c}<T<\sqrt{2 p-1} T_{c}$. Podemos então calcular $\langle\ln Z\rangle$ usando (B.4) e $\lambda=\sqrt{N} \beta J$

$$
\begin{aligned}
\langle\ln Z\rangle= & N \ln 2+\frac{N J^{2}}{4 T^{2}}-\int_{0}^{\infty} d \phi e^{-\phi} Q_{p}\left(\phi / 2^{N}, e^{\lambda^{2} / 4}\right)+\frac{2 \sqrt{\pi} T}{J\left(\frac{T^{2}}{T_{c}^{2}}+1\right) \sqrt{N} \sin \left[\frac{\pi}{2}\left(\frac{T^{2}}{T_{c}^{2}}+1\right)\right]} \\
& \times \exp \left[-\frac{N T^{2} J^{2}}{16}\left(\frac{1}{T_{c}^{2}}-\frac{1}{T^{2}}\right)^{2}\right]+\cdots
\end{aligned}
$$

O termo $\sin \pi\left[\left(T_{c} / T\right)^{2}+1\right] / 2$ vem do produto de duas funções gamma

$$
\Gamma(z) \Gamma(-z)=-\frac{\pi}{\sin \pi z}
$$

A integral do polinômio $Q$ fornece um polinômio de duas variáveis $2^{-N}$ e $e^{N J^{2} / 4 T^{2}}$. O fato importante é que este termo é exponencialmente pequeno em $N$ e é regular em toda temperatura $T_{n}^{\prime}=T_{c} \sqrt{2 n+1}$. Por outro lado o último termo fornece a expansão assintótica (B.42) de $\langle\ln Z\rangle$ que é singular na temperatura mesmo se esta decresce também exponencialmente com $N$. Para $n=1,2$ e 3 podemos expressar (B.42) como:

$$
\begin{aligned}
\langle\ln Z\rangle= & N\left(\ln 2+\frac{J^{2}}{4 J^{2}}\right)-\frac{1}{2}\left\langle\left(\frac{Z}{\langle Z\rangle}-1\right)^{2}\right\rangle+\frac{1}{3}\left\langle\left(\frac{Z}{\langle Z\rangle}-1\right)^{3}\right\rangle \\
& +\cdots+\frac{(-1)^{n+1}}{n}\left\langle\left(\frac{Z}{\langle Z\rangle}-1\right)^{n}\right\rangle+\frac{2 \sqrt{\pi} T}{J\left(\frac{T^{2}}{T_{c}^{2}}+1\right) \sqrt{N} \sin \left[\frac{\pi}{2}\left(\frac{T^{2}}{T_{c}^{2}}+1\right)\right]} \\
& \times \exp \left[-\frac{N T^{2} J^{2}}{16}\left(\frac{1}{T_{c}^{2}}-\frac{1}{T^{2}}\right)^{2}\right]+\cdots
\end{aligned}
$$

Para $\sqrt{2 n-1} T_{c}<T<\sqrt{2 n+1} T_{c}$. 


\section{Apêndice $C$}

\section{Cálculo alternativo da energia livre}

\section{sem o uso de réplicas}

Neste apêndice, consideramos o cálculo alternativo da energia livre do REM ferromagnético na presença de um campo aleatório. As variáveis de spins são do tipo Ising $S_{i}= \pm 1$ e as interações de troca satisfazem a uma distribuição de probabilidade normal. A abordagem utilizada aqui foi empregada por (Haddad, Vieira, e Salinas, 2004) para estudar modelos ferromagnéticos com interações (uniforme) de $p$ spins na presença de um campo aleatório.

A hamiltoniana do sistema é definida como

$$
\mathcal{H}=-\sum_{1 \leq i_{1}<\cdots<i_{p} \leq N} J_{i_{1} \cdots i_{p}} S_{i_{1}} \cdots S_{i_{p}}-\frac{J_{0} N}{2} m^{2}-\sum_{i=1}^{N} H_{i} S_{i}
$$

em que $J_{i_{1} \cdots i_{p}}$ são variáveis aleatórias independentes e identicamente distribuídas com distribuição de probabilidade normal, ou seja, $J_{i_{1} \cdots i_{p}} \sim N\left(0, J^{2} p ! / 2 N^{p-1}\right)$ e $H_{i}$ pode apresentar qualquer distribuição.

A função de partição é dada por

$$
Z=\sum_{S} e^{-\beta \mathcal{H}}, \quad \text { com } \quad \sum_{S} \equiv \sum_{S_{1}=-S}^{S} \cdots \sum_{S_{N}=-S}^{S} .
$$

em que $\beta=1 / k_{B} T$. 
A função de partição pode ser escrita ainda da seguinte forma

$$
\begin{aligned}
Z= & \int_{-\infty}^{\infty} d E_{p} \int_{-\infty}^{\infty} d E_{c} \int_{-\infty}^{\infty} d m \exp \left[-N \beta\left(\frac{E_{p}}{N}+E_{c}-\frac{J_{0} m^{2}}{2}\right)\right] \sum_{S} \delta\left(E_{p}-\mathcal{H}_{p}\right) \delta\left(N E_{c}-\mathcal{H}_{c}\right) \\
& \times \delta\left(N m-\sum_{i=1}^{N} S_{i}\right) \\
= & \int_{-\infty}^{\infty} d E_{p} \int_{-\infty}^{\infty} d E_{c} \int_{-\infty}^{\infty} d m \Omega\left(E_{p}, E_{c}, M\right) \exp \left[-N \beta\left(\frac{E_{p}}{N}+E_{c}-\frac{J_{0} m^{2}}{2}\right)\right],
\end{aligned}
$$

em que definimos a densidade de estados

$$
\Omega\left(E_{p}, E_{c}, M\right)=\sum_{S}\left[\delta\left(E_{p}-\mathcal{H}\right) \delta\left(E_{c}-\mathcal{H}_{c}\right) \delta\left(M-\sum_{i=1}^{N} S_{i}\right)\right] \sim e^{N \phi} .
$$

O cálculo da função de partição fica reduzido a contagem dos estados acessíveis ao sistema fixados $E_{p}, E_{c}$ e $M$.

$$
Z=\int_{-\infty}^{\infty} d E_{p} \int_{-\infty}^{\infty} d E_{c} \int_{-\infty}^{\infty} d m \exp \left[-N \beta\left(\frac{E_{p}}{N}+E_{c}-\frac{J_{0} m^{2}}{2}-\beta^{-1} \phi\right)\right]
$$

em que $\phi$ está associado à densidade de estados pela (C.4) . A magnetização $m$ é definida de maneira usual $N m=\sum_{i} S_{i}$. Podemos definir novas variáveis $\mathcal{H}_{p}$ e $\mathcal{H}_{c}$ como

$$
\mathcal{H}_{p}=-\sum_{1 \leq i_{1}<\cdots<i_{p} \leq N} J_{i_{1} \cdots i_{p}} S_{i_{1}} \cdots S_{i_{p}} \quad \text { e } N \mathcal{H}_{c}=-\sum_{i} H_{i} S_{i}
$$

Cálculo do valor esperado de $\Omega\left(E_{p}, E_{c}, M\right)$ com relação a distribuição de desordem das interações de troca

$$
\left\langle\Omega\left(E_{p}, E_{c}, M\right)\right\rangle_{J}=\sum_{S}\left\langle\delta\left(E_{p}-\mathcal{H}_{p}\right)\right\rangle_{J} \delta\left(N E_{c}-\mathcal{H}_{c}\right) \delta\left(N m-\sum_{i=1}^{N} S_{i}\right)
$$

Sabemos que a distribuição de probabilidade para os níveis de energia $p(E)$ em um dada configuração é dada por

$$
p(E)=\left\langle\delta\left(E_{p}-\mathcal{H}_{p}\right)\right\rangle_{J}=\frac{1}{2 \pi} \int_{-\infty}^{\infty} d k e^{i k E_{p}}\left\langle e^{-i k \mathcal{H}_{p}}\right\rangle_{J}=\frac{1}{\sqrt{\pi N J^{2}}} e^{-E_{p}^{2} / N J^{2}}
$$

Portanto,

$$
\begin{aligned}
\left\langle\Omega\left(E_{p}, E_{c}, M\right)\right\rangle_{J} & =\sum_{S}\left[\left\langle\delta\left(E_{p}-\mathcal{H}_{p}\right)\right\rangle_{J} \delta\left(E_{c}-\mathcal{H}_{c}\right) \delta\left(N m-\sum_{i=1}^{N} S_{i}\right)\right] \\
& =p(E) \sum_{S}\left[\delta\left(N E_{c}-\mathcal{H}_{c}\right) \delta\left(N m-\sum_{i=1}^{N} S_{i}\right)\right] .
\end{aligned}
$$


Novamente, utilizando uma representação integral para a função delta, temos

$$
\begin{aligned}
\sum_{S}\left[\delta\left(N E_{c}-\mathcal{H}_{c}\right) \delta\left(N m-\sum_{i=1}^{N} S_{i}\right)\right]= & \int_{-i \infty}^{i \infty} \int_{-i \infty}^{i \infty} \frac{d \lambda_{1} d \lambda_{2}}{4 \pi^{2}} \exp \left[N\left(\lambda_{1} E_{c}+\lambda_{2} m\right)\right. \\
& \left.\times \frac{1}{N} \sum_{S} \exp \left(\lambda_{1} H_{i}-\lambda_{2}\right) \sum_{i} S_{i}\right]
\end{aligned}
$$

Pela lei dos grandes números, temos com probabilidade 1

$$
\frac{1}{N} \sum_{S} \exp \left[\sum_{i}\left(\lambda_{1} H_{i}-\lambda_{2}\right) S_{i}\right]=\frac{1}{N} \sum_{i=1}^{N} \ln 2 \cosh \left(\lambda_{1} H_{i}-\lambda_{2}\right)=\left\langle\ln 2 \cosh \left(\lambda_{1} H-\lambda_{2}\right)\right\rangle_{H} .
$$

Deste modo,

$$
\begin{aligned}
\sum_{S}\left[\delta\left(N E_{c}-\mathcal{H}_{c}\right) \delta\left(N m-\sum_{i=1}^{N} S_{i}\right)\right]= & \int_{-i \infty}^{i \infty} \int_{-i \infty}^{i \infty} \frac{d \lambda_{1} d \lambda_{2}}{4 \pi^{2}} \exp \left\{N \left[\left(\lambda_{1} E_{c}+\lambda_{2} m\right)\right.\right. \\
& \left.\left.\times\left\langle\ln 2 \cosh \left(\lambda_{1} H-\lambda_{2}\right)\right\rangle_{H}\right]\right\}
\end{aligned}
$$

No limite termodinâmico, $N \rightarrow \infty$, a integral acima pode ser feita pelo método do ponto de sela

$$
\sum_{S}\left[\delta\left(N E_{c}-\mathcal{H}_{c}\right) \delta\left(N m-\sum_{i=1}^{N} S_{i}\right)\right] \sim \exp \left\{N \max _{\lambda_{1}, \lambda_{2}}\left[\lambda_{1} E_{c}+\lambda_{2} m+\left\langle\ln 2 \cosh \left(H \lambda_{1}-\lambda_{2}\right)\right\rangle_{H}\right]\right\},
$$

com as equações de ponto de sela são dadas por

$$
\begin{aligned}
& E_{c}=-\left\langle H \tanh \left(\lambda_{1} H-\lambda_{2}\right)\right\rangle_{H} \\
& m=\left\langle\tanh \left(\lambda_{1} H-\lambda_{2}\right)\right\rangle_{H} .
\end{aligned}
$$

A função densidade de estados é dada por

$$
\left\langle\Omega\left(E_{p}, E_{c}, m\right)\right\rangle_{J}=p(E) \exp \left\{N\left[\lambda_{1} E_{c}+\lambda_{2} m+\left\langle\ln 2 \cosh \left(\lambda_{1} H-\lambda_{2}\right)\right\rangle_{H}\right]\right\}
$$

com $p(E)$ dado por (C.8). Devido a propriedade de self-averging da densidade de estados $\Omega\left(E_{p}, E_{c}, m\right)$, temos

$$
\left\langle\Omega\left(E_{p}, E_{c}, m\right)\right\rangle_{J}=\Omega\left(E_{p}, E_{c}, m\right), \quad \text { com a condição } S=k_{B} \ln \Omega\left(E_{p}, E_{c}, m\right) \geq 0 \text {. (C.17) }
$$

O prodecidimento utilizado para calcular a função de partição (ensemble canônico) envolve uma contagem dos estados do sistema (degenerescência) que é uma abordagem típica 
quando se trabalha no ensemble microcanônico. Desta forma, o modelo é resolvido sem recorrer ao truque de réplicas.

A função de partição (C.2) pode ser escrita como

$$
Z=\int d E_{p} E_{c} d M e^{-\beta F}
$$

Segue que

$$
\begin{aligned}
Z= & \int_{-\infty}^{\infty} d E_{p} \int_{-\infty}^{\infty} d E_{c} \int_{-\infty}^{\infty} d m \exp \left\{-N \beta\left[\frac{E_{p}}{N}+E_{c}-\frac{J_{0} m^{2}}{2}+\beta^{-1}\left(\frac{E_{p}^{2}}{(N J)^{2}}-\lambda_{1} E_{c}\right.\right.\right. \\
& \left.\left.\left.-\lambda_{2} m-\left\langle\ln 2 \cosh \left(\lambda_{1} H-\lambda_{2}\right)\right\rangle_{H}\right)\right]\right\} .
\end{aligned}
$$

No limite termodinâmico, $N \rightarrow \infty$, a integral (C.19) pode ser feita pelo método de Laplace $\ln Z=-N \min _{E_{p}, E_{c}, m}\left[\frac{E_{p}}{N}+E_{c}-\frac{J_{0} m^{2}}{2}+\beta^{-1}\left(\frac{E_{p}^{2}}{N J^{2}}-\lambda_{1} E_{c}-\lambda_{2} m-\left\langle\ln 2 \cosh \left(\lambda_{1} H-\lambda_{2}\right)\right\rangle_{H}\right)\right]$

com as equações de ponto estacionário dadas por

$$
\begin{aligned}
& E_{p}=-\frac{N \beta J^{2}}{2} \\
& \lambda_{1}=\beta \\
& \lambda_{2}=-\beta J_{0} m .
\end{aligned}
$$

Substituindo as equaçōes de ponto de sela em (C.20), temos que a energia livre por spin é dada por

$$
f=-\frac{\beta J^{2}}{4}+\frac{J_{0} m^{2}}{2}-\beta^{-1}\left\langle\ln 2 \cosh \beta\left(H+J_{0} m\right)\right\rangle_{H}
$$

que é exatamente o resultado obtido nos capítulos 2 e 3 .

A entropia é

$$
\frac{s}{k_{B}}=-\frac{(\beta J)^{2}}{4}-\beta J_{0} m^{2}+\left\langle\ln 2 \cosh \left(\beta\left(H+J_{0} m\right)\right\rangle_{H}-\beta\left\langle H \tanh \left(\beta\left(H+J_{0} m\right)\right\rangle_{H}\right.\right.
$$

Lembrando que esses resultados são válidos para $\beta<\beta_{c}$, ou seja, $\ln \Omega\left(E_{p}, E_{c}, m\right) \geq 0$.

A versão antiferromagnética pode ser obtida de maneira análoga, embora com um pouco mais de esforço algébrico. 


\section{Referências Bibliográficas}

Aharony, A. (1978). "Spin-flop multicritical points in systems with random fields and in spin-glasses". Phys. Rev. B, 18(7), 3318-3336.

Almeida, J. R. L. de. (1998). "Magnetic properties of the antiferromagnetic random energy model". Phys. Status Solidi B, 209(1), 153-159.

Almeida, J. R. L. de. (1999). "Susceptibilities of the antiferromagnet REM model". Eur. Phys. J. B, 8(2), 269-273.

Almeida, J. R. L. de, e Bruinsma, R. (1987). "Replica symmetry-breaking in random-field systems". Phys. Rev. B, 35(13), 7267-7270.

Almeida, J. R. L. de, e Thouless, D. J. (1978). "Stability of the Sherrington - Kirkpatrick solution of a spin glass model". J. Phys. A, 11(5), 983-990.

Amit, D. J. (1989). Modeling brain function. Cambrigde University Press.

Anderson, P. W. (1958). "Absence of diffusion in certain random lattices". Phys. Rev., $109(5), 1492-1505$.

Anderson, P. W. (1988). "Spin glass III: Theory raises its head". Phys. Today, 41(6), $9-11$.

Araújo, J. M. de, Costa, F. A. da, e Nobre, F. D. (2000). "First-order transitions and triple point on a random $p$-spin interaction model". J. Phys. A-Math. Gen., 33(10), $1987-1992$.

Ashcroft, N., e Mermin, N. D. (1976). Solid state physics. Harcourt Brace College Publishers. 
Belanger, D. P. (1997). "Experiments on random field Ising model". In A. P. Young (Ed.), Spin glasses and random fields (pp. 251-275). Singapore: World Scientific.

Bertrand, D., Fert, A. R., Schmidt, M. C., Bensamka, F., e Legrand, S. (1982). "Observation of a spin glass-like behavior in dilute system $\mathrm{Fe}_{1-x} \mathrm{Mg}_{x} \mathrm{Cl}_{2}$ ". Journal of Physics C-Solid State Physics, 15(25), L883-L888.

Binder, K., e Young, A.P. (1986). "Spin glasses: Experimental facts, theoretical concepts, and open questions". Rev. Mod. Phys., 58(4), 801-976.

Blandin, A., Gabay, M., e Garel, T. (1980). "Mean-field theory of spin-glasses". Journal of Physics C-Solid State Physics, 13(3), 403-418.

Bricmont, J., e Kupiainen, A. (1987). "Lower critical dimension for the random-field Ising-model". Phys. Rev. Lett., 59(16), 1829-1832.

Brout, R. (1959). "Statistical mechanical theory of a random ferromagnetic system". Phys. Rev., 115(4), 824-835.

Cannella, V., e Mydosh, J. A. (1972). "Magnetic ordering in gold-iron alloys". Phys. Rev. $B, \mathbf{1 1}(6), 4220-4237$.

Cardy, J. L. (1984). "Random-field effects in site-disordered Ising antiferromagnets". Phys. Rev. B, 29(1), 505-507.

Derrida, B. (1980). "Random-energy model: Limit of a family of disordered models". Phys. Rev. Lett., 45(2), 79-82.

Derrida, B. (1981). "Random-energy model: An exactly solvable model of disordered systems". Phys. Rev. B, 24(5), 2613-2626.

Dominicis, C. de, e Kondor, I. (1983). "Eigenvalues of the stability matrix for Parisi solution of the long-range spin-glass". Phys. Rev. B, 27(1), 606-608.

Dominicis, C. de, Kondor, I., e Temesvari, T. (1993). "Ising spin-glass - recent progress in the field-theory approach". International Journal of Modern Physics, 7(1-3), 986992. 
Dorlas, T. C., e Wedagedera, J. R. (1999). "Phase diagram of the random energy model. with higher-order ferromagnetic term and error correcting codes due to Sourlas". Phys. Rev. Lett., 83(21), 4441-4444.

Dotsenko, V. (1994). An introduction to the theory of spin glasses and neural networks. Singapore: World Scientific.

Edwards, S. F., e Anderson, P. W. (1975). "Theory of spin glasses". J. Phys. F: Metal Phys., 5, 965-974.

Ferreira, I. B., King, A. R., Jaccarino, V., e Cardy, J. L. (1983). "Random-fieldinduced destruction of the phase-transition of a diluted two-dimensional Ising antiferromagnet - $\mathrm{Rb}_{2} \mathrm{Co}_{0.85} \mathrm{Mg}_{0.15} \mathrm{~F}_{4}$ ". Phys. Rev. B, 28(9), 5192-5198.

Fischer, K. H., e Hertz, J. H. (1991). Spin glasses. Cambridge: Cambridge University Press.

Fisher, D. S., Grinstein, G. M., e Khurana, A. (1988). "Theory of random magnets". Phys. Today, 41(12), 56-67.

Fisher, D. S., e Huse, D. A. (1986). "Ordered phase of short-range Ising spin-glasses". Phys. Rev. Lett., 56(15), 1601-1604.

Fishman, S., e Aharony, A. (1979). "Random field effects in disordered anisotropic antiferromagnets". Journal of Physics C-Solid State Physics, 12(18), L729-L733.

Gardner, E. (1985). "Spin-glasses with p-spin interactions". Nucl. Phys. B, 257(6), $747-765$.

Gillin, P., Nishimori, H., e Sherrington, D. (2001). "Multispin Ising spin glasses with ferromagnetic interactions". J. Phys. A-Math. Gen., 34(14), 2949-2964.

Gillin, P., e Sherrington, D. (2000). " $p>2$ spin glasses with first-order ferromagnetic transitions". J. Phys. A-Math. Gen., 33(16), 3081-3091.

Gillin, P., e Sherrington, D. (2001). "Comment on 'replica analysis of the $p$-spin interaction Ising spin-glass model"'. J. Phys. A-Math. Gen., 34(6), 1219-1222. 
Gofman, M., Adler, J., Aharony, A., Harris, A. B., e Schwartz, M. (1996). "Critical behavior of the random-field Ising model". Phys. Rev. B, 53(10), 6362-6384.

Gross, D. J., Kanter, I., e Sompolinsky, H. (1985). "Mean-field theory of the Potts glass". Phys. Rev. Lett., 55(3), 304-307.

Gross, D. J., e Mézard, M. (1984). "The simplest spin-glass". Nucl. Phys. B, 240(4), 431-452.

Haddad, T. A. S., Vieira, A. P., e Salinas, S. R. (2004). "Some remarks on p-spin interaction models in a random field". Physica A, 342(1-2), 76-82.

Hemmen, J. L. van, e Palmer, R. G. (1979). "Replica method and a solvable spin glass model". J. Phys. A: Math. Gen., 12(4), 563-580.

Höchli, U. T. (1982). "Dynamics of freezing electric dipoles". Phys. Rev. Lett., 48(21), 1494-1497.

Imbrie, J. Z. (1984). "Lower critical dimension of the random-field Ising-model". Phys. Rev. Lett., 53(18), 1747-1750.

Imry, Y., e Ma, S. (1975). "Random-field instability of ordered state of continuous symmetry". Phys. Rev. Lett., 35(21), 1399-1401.

James, B. R. (1981). Probabilidade: um curso em nível intermediário. Rio de Janeiro: Instituto de Matemática Pura e Aplicada.

Kac, M. (1968). In Trondheim theoretical physics seminar. Nordita, Publ. No. 286.

Kirkpatrick, S., e Thirumalai, D. (1987a). "p-spin-interaction spin-glass models - connections with the structural glass problem". Phys. Rev. B, 36(10), 5388-5397.

Kirkpatrick, S., e Thirumalai, D. (1987b). "Dynamics of the structural glass-transition and the $p$-spins-interaction spin-glass model". Phys. Rev. Lett., 58(20), 2091-2094.

Levstik, A., Filipič́, C., Kutnjak, Z., Levstik, R., Pirc, R., Tadić, B., e Blinc, R. (1991). "Field-cooled and zero-field-cooled dielectric susceptibility in deuteron glasses". Phys. Rev. Lett., 66(18), 2368-2371. 
Ma, Y. Q., Gong, C. D., e Li, Z. Y. (1991). "Reentrant transition in the Ising spin system with an infinite-range coupling and a gaussian random field". Phys. Rev. B, 43(10), $8665-8667$.

Mathews, J., e Walker, R. (1973). Mathematical methods of physics (Second ed.). California: W. A. Benjamin, Inc.

Mazo, R. M. (1963). "Free energy of a system with random elements". J. Chem. Phys., $39(5), 1224-1225$.

Mydosh, J. A. (1993). Spin glasses: An experimental introduction. London: Taylor \& Francis.

Mézard, M., e Monasson, R. (1994). "Glassy transition in the 3-dimensional random-field Ising-model". Phys. Rev. B, 50(10), 7199-7202.

Mézard, M., Parisi, G., e Virasoro, M. A. (1987). Spin glass theory and beyond. Singapore: World Scientific.

Mézard, M., e Young, A. P. (1992). "Replica symmetry-breaking in the random field Ising-model". Europhys Lett., 18(7), 653-659.

Newman, M. E. J., e Barkema, G. T. (1996). "Monte Carlo study of the random-field Ising model". Phys. Rev. E, 53(1), 393-404.

Nogueira, E., Nobre, F. D., Costa, F. A. da, e Coutinho, S. (1998). "Trícritical behavior in the Sherrington-Kirkpatrick spin glass under a bimodal random field". Phys. Rev. $E, \mathbf{5 7}(5), 5079-5086$.

Nogueira, E., Nobre, F. D., Coutinho, S., e Almeida, J. R. L. de. (1996). "m-vector spin glass in the presence of a gaussian random field". Phys. Rev. E, 53(6), 5742-5748.

Oliveira, V. M. de, e Fontanari, J. F. (1999). "Landscape statistics of the $p$-spin Ising model". J. Phys. A-Math. Gen, 32(24), 8445-8457.

Parisi, G. (1979). "Toward a mean field-theory for spin-glasses". Phys. Lett. A, 73(3), 203-205. 
Parisi, G. (1980a). "Order parameter for spin-glasses - function on the interval 0-1". $J$. Phys. A: Math. Gen., 13(3), 1101-1112.

Parisi, G. (1980b). "A sequence of approximated solutions to the S-K model for spinglasses". J. Phys. A: Math. Gen., 13(4), L115-L121.

Pirc, R., Blinc, R., e Wiotte, W. (1992). "Correlated random bonds and random fields in proton glasses". Physica B, 182(2), 137-144.

Pirc, R., Tadić, B., e Blinc, R. (1987). "Random-field smearing of the proton-glass transition". Phys. Rev. B, 36(16), 8607-8615.

Queiroz Jr., I. S., Costa, F. A. F. da, e Nobre, F. D. (2002). "A p-spin interaction Ashkin-Teller spin-glass model”. Physica A, 309(9), 106-120.

Salinas, S. R., e Wreszinski, W. F. (1985). "On the mean-field Ising-model in a random external-field". J. Stat. Phys., 41(1-2), 299-313.

Shapir, Y. (1992). In recent progress in random magnets. Singapore: World Scientific.

Sherrington, D., e Kirkpatrick, S. (1975). "Solvable model of a spin-glass". Phys. Rev. Lett., $\mathbf{3 5}(26)$, 1972-1976.

Soares, R. F., Nobre, F. D., e Almeida, J. R. L. de. (1994). "Effects of a gaussian randomfield in the Sherrington-Kirkpatrick spin-glass". Phys. Rev. B, 50(9), 6151-6156.

Toulouse, G. (1977). "Theory of frustration effect in spin-glasses: I.". Comm. Phys., $2(4), 115-119$.

Vieira, S. R., Nobre, F. D., e Yokoi, C. S. O. (2000). "Effects of random fields in an antiferromagnetic Ising spin glass". Phys. Rev. E, 61(5), 4760-4768.

Wong, P. zen, Molnar, S. von, Palstra, T. T. M., Mydosh, J. A., Yoshizawa, H., Shapiro, S. M., e Ito, A. (1985). "Coexistence of spin-glass and antiferromagnetic orders in the Ising system $\mathrm{Fe}_{0.55} \mathrm{Mg}_{0.45} \mathrm{Cl}_{2}$. Phys. Rev. Lett., 55(19), 2043-2046. 
Wong, P. zen, Yoshizawa, H., e Shapiro, S. M. (1985). "Coexistence of antiferromagnetism and spin-glass ordering in the Ising system $\mathrm{Fe}_{0.55} \mathrm{Mg}_{0.45} \mathrm{Cl}_{2}$ ". Journal of Applied Physics, 57(8), 3462-3464.

Yoshizawa, H., Mitsuda, S., Aruga, H., e Ito, A. (1987). "Mixed phase of spin-glass ordering and antiferromagnetism in an Ising system, $\mathrm{Fe}_{x} \mathrm{Mn}_{1-x} \mathrm{TiO}_{3} "$. Phys. Rev. Lett., $59(20), 2364-2367$.

Young, A. P. (Ed.). (1997). Spin glasses and random fields. Singapore: World Scientific. 